\title{
An Integrated Version of Varadhan's Asymptotics for Lower-Order Perturbations of Strong Local Dirichlet Forms
}

\author{
Masanori Hino $^{1} \cdot$ Kouhei Matsuura $^{2}$
}

Received: 30 March 2017 / Accepted: 9 June 2017 / Published online: 22 June 2017

(C) The Author(s) 2017. This article is an open access publication

\begin{abstract}
The studies of J. A. Ramírez, Hino-Ramírez, and Ariyoshi-Hino showed that an integrated version of Varadhan's asymptotics holds for Markovian semigroups associated with arbitrary strong local symmetric Dirichlet forms. In this paper, we consider nonsymmetric bilinear forms that are the sum of strong local symmetric Dirichlet forms and lower-order perturbed terms. We give sufficient conditions for the associated semigroups to have asymptotics of the same type.
\end{abstract}

Keywords Varadhan's asymptotics · Short-time behavior · Dirichlet form · Intrinsic distance

Mathematics Subject Classification (2010) 31C25 - 60J60 - 58J37 · 47D07

\section{Introduction}

Let $(E, \mathscr{B}, \mu)$ be a $\sigma$-finite measure space and $\left(\mathscr{E}^{0}, \mathbb{D}\right)$ a symmetric strong local Dirichlet form on the $L^{2}$ space of $(E, \mathscr{B}, \mu)$. Let $\left\{T_{t}^{0}\right\}_{t>0}$ denote the semigroup associated with $\left(\mathscr{E}^{0}, \mathbb{D}\right)$, and set $P_{t}^{0}(A, B)=\int_{A} T_{t}^{0} \mathbf{1}_{B} d \mu$ for $t>0$ and $A, B \in \mathscr{B}$ with positive and finite

Dedicated to Professor Masayoshi Takeda on the occasion of his 60th birthday

Masanori Hino

hino@math.kyoto-u.ac.jp

Kouhei Matsuura

kouhei.matsuura.r3@dc.tohoku.ac.jp

1 Department of Mathematics, Kyoto University, Kyoto 606-8502, Japan

2 Mathematical Institute, Tohoku University, Aoba, Sendai 980-8578, Japan 
measure. In [1], the following small-time asymptotic estimate for $\left\{T_{t}^{0}\right\}_{t>0}$ was proved as a generalization of results from previous work $[8,9,16]$ :

$$
\lim _{t \rightarrow 0} t \log P_{t}^{0}(A, B)=-\frac{\mathrm{d}(A, B)^{2}}{2} .
$$

Here, $\mathrm{d}(A, B)$ is the intrinsic distance between $A$ and $B$, which can be determined from only $\left(\mathscr{E}^{0}, \mathbb{D}\right)$ (see $[1$, p. 1241$]$ or Definition 2.6 below for details). Similar small-time asymptotics of transition densities have been studied extensively. These are usually called Varadhan-type estimates, in reference to [19]. In particular, that the estimate holds was proved in [15] for a class of symmetric and uniform elliptic diffusion processes on Lipschitz manifolds. This is one of the most general results. Asymptotics of the form Eq. 1.1 can be considered as an integrated version of Varadhan's asymptotics.

The purpose of this paper is to extend the formula (1.1) to a class of non-symmetric bilinear forms. Specifically, we first assume that $(\mathscr{E} 0, \mathbb{D})$ mentioned above is expressed as

$$
\mathscr{E}^{0}(f, g)=\frac{1}{2} \int_{E}(D f, D g)_{H} d \mu, \quad f, g \in \mathbb{D},
$$

where $D$ is a first-order derivation operator taking values in a separable Hilbert space $H$. Our main object is to obtain small-time asymptotics for a non-symmetric form $(\mathscr{E}, \mathbb{D})$ given by the sum of $\mathscr{E}^{0}$ and the lower-order term $\int_{E}(b, D f)_{H} g d \mu+\int_{E}(c, D g)_{H} f d \mu+$ $\int_{E} V f g d \mu$ (see Eq. 2.4). Since Varadhan's original paper [19] treats some non-symmetric cases, this generalization is natural. When the lower-order term is small relative to $\mathscr{E} 0$, the form $(\mathscr{E}, \mathbb{D})$ becomes a lower-bounded bilinear form and has an associated positivitypreserving semigroup $\left\{T_{t}\right\}_{t>0}$ on $L^{2}(E, \mu)$. For measurable sets $A$ and $B$ having positive and finite $\mu$-measure, let $P_{t}(A, B)=\int_{A} T_{t} \mathbf{1}_{B} d \mu$, as before. We study the conditions on $b, c$, and $V$ that suffice for the semigroup $\left\{T_{t}\right\}_{t>0}$ to have the same integrated Varadhan's asymptotics as $\left\{T_{t}^{0}\right\}_{t>0}$. That is, for

$$
\lim _{t \rightarrow 0} t \log P_{t}(A, B)=\lim _{t \rightarrow 0} t \log P_{t}^{0}(A, B)=-\frac{\mathrm{d}(A, B)^{2}}{2} .
$$

This looks generically true at first glance, but if $b, c$, and $V$ are unbounded, it is a very nontrivial problem to reveal what kind of restrictions we should impose on them to guarantee the validity of Eq. 1.2.

It is reasonable to expect that Eq. 1.2 would hold if they were sufficiently smaller than $\mathscr{E}^{0}$ in terms of quadratic forms. From another perspective, we can make a probabilistic argument, exemplified in the following typical case. Let $(E, H, \mu)$ be an abstract Wiener space, and suppose that $(\mathscr{E} 0, \mathbb{D})$ and $(\mathscr{E}, \mathbb{D})$ are defined as

$$
\begin{aligned}
\mathscr{E}^{0}(f, g) & =\frac{1}{2} \int_{E}(D f, D g)_{H} d \mu, \\
\mathscr{E}(f, g) & =\mathscr{E}^{0}(f, g)+\int_{E}(b, D f)_{H} g d \mu, \quad f, g \in \mathbb{D}:=\mathbb{D}^{1,2},
\end{aligned}
$$

where $D$ denotes the $H$-derivative in the Malliavin calculus, $b$ is an $H$-valued measurable function on $E$, and $\mathbb{D}^{1,2}$ is the first-order $L^{2}$-Sobolev space on $E$. If $\exp \left(\gamma|b|_{H}^{2}\right)$ is $\mu$-integrable for some $\gamma>8$, then by using the logarithmic Sobolev inequality, we can prove that $(\mathscr{E}, \mathbb{D})$ is well-defined as a lower-bounded bilinear form and that there exists a 
corresponding semigroup $\left\{T_{t}\right\}_{t>0}$ on $L^{2}(E, \mu)$ (see Example 5.5). Moreover, $\left\{T_{t}\right\}_{t>0}$ has a probabilistic representation as

$$
T_{t} f(x)=\mathbb{E}_{x}\left[f\left(X_{t}\right) \exp \left(M_{t}-\frac{1}{2}\langle M\rangle_{t}\right)\right],
$$

where $\left(\left\{X_{t}\right\}_{t \geq 0},\left\{\mathbb{P}_{x}\right\}_{x \in E}\right)$ is the Ornstein-Uhlenbeck process associated with $\left(\mathscr{E}^{0}, \mathbb{D}\right)$ and $\left\{M_{t}\right\}_{t \geq 0}$ is a martingale additive functional suitably associated with $b$ (see, e.g., [7]). Note, in particular, that the quadratic variation of $M$ is given by $\langle M\rangle_{t}=\int_{0}^{t}\left|b\left(X_{s}\right)\right|_{H}^{2} d s$. From Hölder's inequality, for measurable sets $A$ and $B$ with positive $\mu$-measure,

$$
\begin{aligned}
\int_{A} T_{t} \mathbf{1}_{B} d \mu \leq & \left(\int_{A} \mathbb{E}_{x}\left[\mathbf{1}_{B}\left(X_{t}\right)\right] d \mu\right)^{1 / p}\left(\int_{A} \mathbb{E}_{x}\left[\exp \left(2 q M_{t}-\frac{1}{2}\langle 2 q M\rangle_{t}\right)\right] d \mu\right)^{1 / 2 q} \\
& \times\left(\int_{A} \mathbb{E}_{x}\left[\exp \left(\left(2 q^{2}-q\right)\langle M\rangle_{t}\right)\right] d \mu\right)^{1 / 2 q}
\end{aligned}
$$

where $p>1$ and $q$ is the conjugate exponent of $p$. The first term of the right-hand side is $P_{t}^{0}(A, B)^{1 / p}$. The second term is dominated by $\mu(A)^{1 / 2 q}$. The third term is estimated by Jensen's inequality:

$$
\begin{aligned}
& \int_{A} \mathbb{E}_{x}\left[\exp \left(\left(2 q^{2}-q\right) \int_{0}^{t}\left|b\left(X_{S}\right)\right|_{H}^{2} d s\right)\right] d \mu \\
& \leq \frac{1}{t} \int_{0}^{t} \int_{A} \mathbb{E}_{x}\left[\exp \left(\left(2 q^{2}-q\right) t\left|b\left(X_{S}\right)\right|_{H}^{2}\right)\right] d \mu d s .
\end{aligned}
$$

By the exponential integrability of $|b|_{H}^{2}$, the right-hand side is equal to

$$
\frac{1}{t} \int_{0}^{t} \int_{A} T_{s}^{0}\left(\exp \left(\left(2 q^{2}-q\right) t|b|_{H}^{2}\right)\right) d \mu d s=\frac{1}{t} \int_{0}^{t} \int_{E}\left(T_{S}^{0} \mathbf{1}_{A}\right) \exp \left(\left(2 q^{2}-q\right) t|b|_{H}^{2}\right) d \mu d s,
$$

which is finite for sufficiently small positive values of $t$ and converges to $\mu(A)$ as $t \rightarrow 0$. Combining these estimates and the asymptotics with respect to $\left(\mathscr{E}^{0}, \mathbb{D}\right)$ and letting $p \rightarrow 1$, we obtain the upper estimate

$$
\varlimsup_{t \rightarrow 0} t \log P_{t}(A, B) \leq-\frac{\mathrm{d}(A, B)^{2}}{2} .
$$

This kind of probabilistic argument is applicable to more general situations, by using a generalized Cameron-Martin-Maruyama-Girsanov formula (see, e.g., [5, 12, 17]). The exponential integrability condition imposed above is not exactly consistent with smallness in the sense of quadratic forms. Indeed, in the estimate of Eq. 1.3, we used the fact that $\exp \left(\gamma|b|_{H}^{2}\right)$ is $\mu$-integrable for only some $\gamma>0$. Therefore, it is reasonable to consider two types of smallness-smallness in term of quadratic forms and in terms of some exponential integrability-in describing the conditions sufficient for Eq. 1.2.

In this paper, we introduce conditions that take the observation above into consideration (see conditions (B.2) ${ }_{A, B},($ B.2' $)$, and Proposition 2.14) and prove the upper estimate under their assumptions (Theorem 2.10). Moreover, we prove that the lower estimate holds under minimal assumptions on $b, c$, and $V$ along with the assumption of the validity of the upper estimate (Theorem 2.11). Combining these two results gives sufficient conditions for the integrated Varadhan estimates. As in the previous studies $[1,9,16]$, the proof is purely analytic and only a measurable structure is imposed on the state space. In particular, we can recapture the probabilistic argument mentioned above by an analytic one. Since the framework is very general and the imposed conditions are mild, our theorems seem novel 
even when $E$ is a Riemannian manifold, not to mention an infinite dimensional space. We also remark that even for $b=c$, that is, even with $(\mathscr{E}, \mathbb{D})$ as a symmetric form, our results are new.

Because the proof is long, we briefly explain the broad ideas of the proof here. The upper estimate (Theorem 2.10) is proved in the spirit of Davies-Gaffney's method. In previous works $[1,9,16]$, they define $\sigma(t)=\int_{E}\left(e^{\alpha w} T_{t} \mathbf{1}_{B}\right)^{2} d \mu$ for given $\alpha>0$ and $w$ with $|D w|_{H} \leq 1 \mu$-a.e., and deduce the key differential inequality $\sigma^{\prime}(t) \leq \alpha^{2} \sigma(t)$. Solving this inequality and optimizing it with respect to $\alpha$ and $w$ yields the desired estimate. Under the assumptions of our theorem, however, the lower-order terms cannot be controlled. Instead, we define $\sigma$ in the form $\sigma(t)=\int_{E}\left(e^{\alpha w} T_{t} \mathbf{1}_{B}\right)^{p(t)} d \mu$, where $p(t)=q-S t$ with $q>2$ and $S>0$ being chosen suitably. Since $\left\{T_{t}\right\}_{t>0}$ can be extended to a semigroup on $L^{p}(\mu)$ for $p$ near 2 in our setting, $\sigma(t)$ is finite for small $t$. The variable exponent $p(t)$ means that the derivative of $\sigma$ involves an extra logarithmic term, which suppresses the influence on $b$ and $c$. The price to pay for this is that the resulting differential inequality is coarser, in the form $\sigma^{\prime}(t) \leq(1+\varepsilon) \alpha^{2} \sigma(t)+C \sigma(t) \max \{0,-\log \sigma(t)\}$. Fortunately, the extra logarithmic term has no influence on the Varadhan-type estimate. Introducing a variable exponent is a standard technique for estimating heat kernel densities (see, e.g., [4]), but (unlike in such a context) $p(t)$ is taken to be a decreasing function in this study. The definition of $\sigma$ shown above is valid when $\mu$ is a finite measure; in general cases, we further need to modify the definition of $\sigma$ (see Eqs. 3.15 and 3.6) to avoid some technical obstacles. For this reason, we need a series of quantitative estimates, which makes the proof long.

The proof of the lower estimate is based on previous studies $[1,9,16]$, but the argument is more complicated due to the perturbed terms and the fact that the semigroup $\left\{T_{t}\right\}_{t>0}$ preserves positivity but is not Markovian. We will outline the proof by the following formal argument. We see the function $u_{t}=-t \log T_{t} \mathbf{1}_{B}$ satisfies the relation

$$
t\left(\partial_{t} u_{t}-\frac{\mathscr{L}^{0}}{2} u_{t}\right)=u_{t}-\frac{1}{2}\left|D u_{t}\right|_{H}^{2}+(\text { extra terms involving } b, c, \text { and } V),
$$

where $\mathscr{L}^{0}$ is the generator of $\left\{T_{t}^{0}\right\}_{t>0}$. (This identity corresponds to Eq. 4.2 in the actual argument.) If we assume for argument that the left-hand side converges to 0 as $t \rightarrow 0$ and the last term of the right-hand side is negligible, then the limit $u_{0}$ of $u_{t}$ (if it exists) will satisfy $\left|D u_{0}\right|_{H}^{2}=2 u_{0}$. What is actually obtained is an inequality of the form $\left|D u_{0}\right|_{H}^{2} \leq 2 u_{0}$, which implies $\left|D \sqrt{2 u_{0}}\right|_{H} \leq 1$. Furthermore, $u_{0}=0 \mu$-a.e. on $B$ should be satisfied. Under these conditions, we have the formal inequality

$$
\lim _{t \rightarrow 0} \sqrt{-2 t \log T_{t} \mathbf{1}_{B}(x)} \leq \mathrm{d}(\{x\}, B)
$$

by the definition of $d$, which is close to the lower-side estimate. Several difficulties, such as that $u_{t}$ is not necessarily bounded, make it hard to justify this procedure directly. To cope with problems such as the integrability (or not) of various terms and the existence (or not) of limits, we introduce a nice truncating function $\phi$ and bump functions $\left\{\chi_{k}\right\}$, and consider $\bar{\phi}_{t} \chi_{k}$ in place of $u_{t}$, where $\bar{\phi}_{t}=t^{-1} \int_{0}^{t} \phi\left(-s \log T_{s} \mathbf{1}_{B}\right) d s$. Note that these cut-off functions are slightly different from those in $[1,9]$ in order to deal with the lack of the Markov property of $\left\{T_{t}\right\}_{t>0}$. This modification results in an increasing number of terms in the quantitative estimates as the proof progresses, which makes the proof longer and more technical than without the modification.

This paper is organized as follows. In Section 2, we introduce a framework and state the main theorems. Section 3 provides the proof of the upper estimate (Theorem 2.10). Section 4 
provides the proof of the lower estimate (Theorem 2.11). In the last section, we prove some auxiliary propositions, discuss the conditions imposed on the theorems, and show some typical examples.

\section{Framework}

Let $(E, \mathscr{B}, \mu)$ be a $\sigma$-finite measure space, and $H$ a real separable Hilbert space. The inner product and norm of $H$ will be denoted by $(\cdot, \cdot)_{H}$ and $|\cdot|_{H}$, respectively. The set of all realvalued measurable functions on $E$ is denoted by $L^{0}(\mu)$, where two functions are identified if they coincide $\mu$-a.e. For $p \in[1, \infty]$, the real $L^{p}$ space on $(E, \mathscr{B}, \mu)$ is denoted by $L^{p}(\mu)$, and its norm by $\|\cdot\|_{p}$. The $L^{2}$ space of $H$-valued measurable functions on $(E, \mathscr{B}, \mu)$ is denoted by $L^{2}(\mu ; H)$, and its norm by $\|\cdot\|_{2}$.

Let $\mathbb{D}$ be a dense subspace of $L^{2}(\mu)$, and $D$ be a closed linear operator from $L^{2}(\mu)$ to $L^{2}(\mu ; H)$ with domain $\mathbb{D}$. We assume that $D$ has the following derivation property: For arbitrary functions $f_{1}, f_{2}, \ldots, f_{m} \in \mathbb{D}$ and $C^{1}$ functions $F$ on $\mathbb{R}^{m}$ with bounded first-order derivatives and $F(0)=0, F\left(f_{1}, \ldots, f_{m}\right)$ belongs to $\mathbb{D}$ and

$$
D\left(F\left(f_{1}, \ldots, f_{m}\right)\right)=\sum_{j=1}^{m} \frac{\partial F}{\partial x_{j}}\left(f_{1}, \ldots, f_{m}\right) D F_{j} .
$$

Then, a bilinear form $\left(\mathscr{E}^{0}, \mathbb{D}\right)$ on $L^{2}(\mu)$, defined by

$$
\mathscr{E}^{0}(f, g)=\frac{1}{2} \int_{E}(D f, D g)_{H} d \mu, \quad f, g \in \mathbb{D},
$$

is a Dirichlet form on $L^{2}(\mu)$. Moreover, this bilinear form has a strong local property: For any $f \in \mathbb{D}$ and $C^{1}$-functions $F, G$ on $\mathbb{R}$ with bounded first-order derivatives such that the supports of $F$ and $G$ are disjoint, $\mathscr{E}(F(f)-F(0), G(f)-G(0))=0$. For other equivalent statements, see [3, Proposition I.5.1.3], where this property is called a local property.

For $l \geq 0$, we write $\mathscr{E}_{l}^{0}(f, g)$ for $\mathscr{E}^{0}(f, g)+l \int_{E} f g d \mu$. We also use $\mathscr{E}^{0}(f)$ and $\mathscr{E}_{l}^{0}(f)$ to denote $\mathscr{E}^{0}(f, f)$ and $\mathscr{E}_{l}^{0}(f, f)$, respectively. The space $\mathbb{D}$ becomes a Hilbert space with the inner product $(f, g) \mapsto \mathscr{E}_{1}^{0}(f, g)$. The following proposition is fundamental.

Proposition 2.1 If a function $f \in \mathbb{D}$ is constant $\mu$-a.e. on a set $A \in \mathscr{B}$, then $D f=0 \mu$-a.e. on A.

Proof Suppose that $f=\alpha \mu$-a.e. on $A$ for some constant $\alpha$. Then, if $\alpha=0$, the conclusion follows from [3, Proposition I.7.1.4]. If $\alpha \neq 0$, we can take a $C^{1}$ function $F$ on $\mathbb{R}$ with bounded derivative such that $F(0)=F(\alpha)=0$ and $F^{\prime}(\alpha) \neq 0$. Then, since $F(f)=0$ $\mu$-a.e. on $A$,

$$
0=D(F(f))=F^{\prime}(f) D f=F^{\prime}(\alpha) D f \quad \mu \text {-a.e. on } A .
$$

This implies that $D f=0 \mu$-a.e. on $A$.

For $A \in \mathscr{B}$, we set

$$
\begin{aligned}
\mathbb{D}_{A} & =\{f \in \mathbb{D} \mid f=0 \mu \text {-a.e. on } E \backslash A\}, \\
\mathbb{D}_{A, b} & =\mathbb{D}_{A} \cap L^{\infty}(\mu), \\
\mathbb{D}_{A, b,+} & =\left\{f \in \mathbb{D}_{A, b} \mid f \geq 0 \mu \text {-a.e. }\right\}
\end{aligned}
$$


We follow [1] in introducing the concept of measurable nests and related function spaces.

Definition 2.2 An increasing sequence $\left\{E_{k}\right\}_{k=1}^{\infty}$ in $\mathscr{B}$ is called a measurable nest ${ }^{1}$ if the following conditions are satisfied.

(i) For every $k \in \mathbb{N}$, there exists $h_{k} \in \mathbb{D}$ such that $h_{k} \geq 1 \mu$-a.e. on $E_{k}$.

(ii) The set $\bigcup_{k=1}^{\infty} \mathbb{D}_{E_{k}}$ is dense in $\mathbb{D}$.

Remark 2.3 We note that measurable nests exist, from [1, Lemma 3.1]. For every $k \in \mathbb{N}$, we have $\mu\left(E_{k}\right)<\infty$ because of condition (i). By condition (ii), $\mu\left(E \backslash \bigcup_{k=1}^{\infty} E_{k}\right)=0$ follows. If $\left\{E_{k}\right\}_{k=1}^{\infty}$ and $\left\{E_{k}^{\prime}\right\}_{k=1}^{\infty}$ are both measurable nests, then so is $\left\{E_{k} \cap E_{k}^{\prime}\right\}_{k=1}^{\infty}$, from [1, Lemma 3.2].

Definition 2.4 For a measurable nest $\left\{E_{k}\right\}_{k=1}^{\infty}$ and $p \in[1, \infty]$, we set

$$
\begin{aligned}
L_{\mathrm{loc}}^{p}\left(\mu,\left\{E_{k}\right\}\right) & =\left\{f \in L^{0}(\mu) \mid f \mathbf{1}_{E_{k}} \in L^{p}(\mu) \text { for every } k \in \mathbb{N}\right\}, \\
\mathbb{D}_{\mathrm{loc}}\left(\left\{E_{k}\right\}\right) & =\left\{f \in L^{0}(\mu) \mid \begin{array}{l}
\text { There exist }\left\{f_{k}\right\}_{k=1}^{\infty} \subset \mathbb{D} \text { such that } f= \\
f_{k} \mu \text {-a.e. on } E_{k} \text { for each } k \in \mathbb{N}
\end{array}\right\}, \\
\mathbb{D}_{\mathrm{loc}, b}\left(\left\{E_{k}\right\}\right) & =\mathbb{D}_{\mathrm{loc}}\left(\left\{E_{k}\right\}\right) \cap L^{\infty}(\mu), \\
\mathbb{D}_{\mathrm{loc}, b,+}\left(\left\{E_{k}\right\}\right) & =\left\{f \in \mathbb{D}_{\mathrm{loc}, b}\left(\left\{E_{k}\right\}\right) \mid f \geq 0 \mu \text {-a.e. }\right\} .
\end{aligned}
$$

We remark that $L^{p}(\mu) \subset L_{\text {loc }}^{p}\left(\mu,\left\{E_{k}\right\}\right) \subset L_{\text {loc }}^{q}\left(\mu,\left\{E_{k}\right\}\right)$ for $1 \leq q \leq p \leq \infty$. For $f \in \mathbb{D}_{\text {loc }}\left(\left\{E_{k}\right\}\right), D f$ is defined as an $H$-valued measurable function on $E$ by $D f=D f_{k}$ on $E_{k}$, where $f_{k} \in \mathbb{D}$ and $f_{k}=f \mu$-a.e. on $E_{k}$. From Proposition 2.1, $D f$ is well-defined up to $\mu$-equivalence.

Definition 2.5 For a measurable nest $\left\{E_{k}\right\}_{k=1}^{\infty}$, we set

$$
\mathbb{D}_{0}\left(\left\{E_{k}\right\}\right)=\left\{\left.f \in \mathbb{D}_{\text {loc }, b}\left(\left\{E_{k}\right\}\right)|| D f\right|_{H} \leq 1 \mu \text {-a.e. }\right\} .
$$

This definition is consistent with [1, Definition 2.6], which considers more general situations. The function space $\mathbb{D}_{0}\left(\left\{E_{k}\right\}\right)$ does not depend on the choice of $\left\{E_{k}\right\}_{k=1}^{\infty}$, from $\left[1\right.$, Proposition 3.9]; we therefore denote it as $\mathbb{D}_{0}$. We now define the intrinsic distance between two sets as follows.

Definition 2.6 (see [1, p. 1241]) For $A, B \in \mathscr{B}$ with positive $\mu$ measures, we define

$$
\mathrm{d}(A, B)=\sup _{f \in \mathbb{D}_{0}}\{\underset{x \in A}{\operatorname{essinf}} f(x)-\underset{x \in B}{\operatorname{ess} \sup } f(x)\} \in[0, \infty],
$$

where the essential infimum ess inf and essential supremum ess sup are taken with respect to $\mu$.

We introduce the concept of a distance-like function $\mathrm{d}_{B}$ from the set $B$ and quote a result from [1]. For $B \in \mathscr{B}$ and $N \geq 0$, define ${ }^{2}$

$$
\mathbb{D}_{B, N}=\left\{f \in \mathbb{D}_{0} \mid f=0 \text { on } B \text { and } 0 \leq f \leq N \mu \text {-a.e. }\right\} .
$$

\footnotetext{
${ }^{1}$ In [1], it is called just a nest.

${ }^{2}$ This definition is slightly different from that in [1], but the difference is unimportant in our context.
} 
For $x, y \in \mathbb{R}$, we let $x \vee y$ and $x \wedge y$ denote $\max \{x, y\}$ and $\min \{x, y\}$, respectively.

Proposition 2.7 ([1, Proposition 3.11]) For each $B \in \mathscr{B}$, there exists a unique $[0,+\infty]$ valued measurable function $\mathrm{d}_{B}$ on $E$ (up to $\mu$-null sets) such that, for every $N>0, \mathrm{~d}_{B} \wedge N$ is the maximal element of $\mathbb{D}_{B, N}: \mathrm{d}_{B} \wedge N \in \mathbb{D}_{B, N}$ and $f \leq \mathrm{d}_{B} \wedge N$ u-a.e. for every $f \in \mathbb{D}_{B, N}$. Moreover, $\mathrm{d}(A, B)=\operatorname{ess}_{\inf _{x \in A}} \mathrm{~d}_{B}(x)$ for every $A \in \mathscr{B}$.

We define $D \mathrm{~d}_{B}$ by $D \mathrm{~d}_{B}=D\left(\mathrm{~d}_{B} \wedge N\right)$ on $\left\{\mathrm{d}_{B} \leq N\right\}$ for $N>0$ and $D \mathrm{~d}_{B}=0$ on $\left\{\mathrm{d}_{B}=\infty\right\}$. This is well-defined, by Proposition 2.1 .

To introduce the lower-order terms, let $b$ and $c$ be $H$-valued measurable functions on $E$, and let $V$ be a real measurable function on $E$. From here, we always assume the following minimal requirement.

Assumption 2.8 (A.1) There exists a measurable nest $\left\{E_{k}\right\}_{k=1}^{\infty}$ such that $|b|_{H},|c|_{H} \in$ $L_{\text {loc }}^{2}\left(\mu,\left\{E_{k}\right\}\right)$ and $V \in L_{\text {loc }}^{1}\left(\mu,\left\{E_{k}\right\}\right)$.

(A.2) There exist $\eta \in[0,1), \theta \geq 0, \omega \geq 0$, and $l \geq 0$ such that, for every $f, g \in$ $\bigcup_{k=1}^{\infty} \mathbb{D}_{E_{k}, b}$,

$$
-\int_{E}\left\{(b+c, D f)_{H} f+V f^{2}\right\} d \mu \leq \eta \mathscr{E}^{0}(f)+\theta\|f\|_{2}^{2}
$$

and

$$
\left|\int_{E}\left\{(b, D f)_{H} g+(c, D g)_{H} f+V f g\right\} d \mu\right| \leq \omega \mathscr{E}_{l}^{0}(f)^{1 / 2} \mathscr{E}_{l}^{0}(g)^{1 / 2} .
$$

For $f, g \in \bigcup_{k=1}^{\infty} \mathbb{D}_{E_{k}, b}$, we define

$$
\mathscr{E}(f, g)=\mathscr{E}^{0}(f, g)+\int_{E}\left\{(b, D f)_{H} g+(c, D g)_{H} f+V f g\right\} d \mu .
$$

It follows that

$$
\begin{aligned}
\mathscr{E}(f, f)+\theta\|f\|_{2}^{2} & =\mathscr{E}^{0}(f)+\int_{E}\left\{(b, D f)_{H} f+(c, D f)_{H} f+V f^{2}\right\} d \mu+\theta\|f\|_{2}^{2} \\
& \geq(1-\eta) \mathscr{E}^{0}(f), \\
|\mathscr{E}(f, g)| & \leq\left|\mathscr{E}^{0}(f, g)\right|+\left|\int_{E}\left\{(b, D f)_{H} g+(c, D g)_{H} f+V f g\right\} d \mu\right| \\
& \leq(1+\omega)_{\mathscr{E}_{l}}(f)^{1 / 2} \mathscr{E}_{l}^{0}(g)^{1 / 2},
\end{aligned}
$$

and $\bigcup_{k=1}^{\infty} \mathbb{D}_{E_{k}, b}$ is dense in $\mathbb{D}$. Therefore, $\mathscr{E}(\cdot, \cdot)$ extends continuously to a bilinear form on $\mathbb{D}$ and the bilinear form $\mathbb{D} \times \mathbb{D} \ni(f, g) \mapsto \mathscr{E}_{\theta}(f, g):=\mathscr{E}(f, g)+\theta \int_{E} f g d \mu$ is a coercive closed form on $L^{2}(\mu)$. Thus, a strongly continuous semigroup $\left\{T_{t}\right\}_{t>0}$ exists on $L^{2}(\mu)$ and some closed operator $(\mathscr{L}, \operatorname{Dom}(\mathscr{L}))$ on $L^{2}(\mu)$ associated with $(\mathscr{E}, \mathbb{D})$ satisfies $\mathscr{E}(f, g)=$ $-\int_{E}(\mathscr{L} f) g d \mu$ for $f \in \operatorname{Dom}(\mathscr{L})$ and $g \in \mathbb{D}$. In particular, $T_{t}$ can be given as $e^{\theta t} T_{t}^{(\theta)}$, where $\left\{T_{t}^{(\theta)}\right\}_{t>0}$ is the semigroup associated with $\left(\mathscr{E}_{\theta}, \mathbb{D}\right)$. Formally, $\mathscr{L}$ is described as

$$
-(1 / 2) D^{*} D-(b, D \cdot)_{H}-D^{*}(c \cdot)-V \cdot,
$$

where $D^{*}$ denotes the adjoint operator of $D$. Confirming that $\left(\mathscr{E}_{\theta}, \mathbb{D}\right)$ satisfies the condition (S) in [14, Proposition 1.2] by an argument similar to [14, Proof of Theorem 2.2] and applying [14, Theorem 1.5], we see that $\left\{T_{t}\right\}_{t>0}$ is positivity preserving. That is, $T_{t} f \geq 0$ $\mu$-a.e. if $f \geq 0 \mu$-a.e. In general, $\left\{T_{t}\right\}_{t>0}$ is not necessarily Markovian. Let $\hat{T}_{t}$ denote the 
adjoint operator of $T_{t}$ on $L^{2}(\mu)$. Then, $\left\{\hat{T}_{t}\right\}_{t>0}$ is also a positivity-preserving semigroup and is associated with a bilinear form $(\hat{\mathscr{E}}, \mathbb{D})$ defined by

$$
\hat{\mathscr{E}}(f, g)=\mathscr{E}(g, f), \quad f, g \in \mathbb{D} .
$$

Let $\mathscr{B}_{0}$ denote the set of all sets $A \in \mathscr{B}$ such that $0<\mu(A)<\infty$. For $A, B \in \mathscr{B}_{0}$, define

$$
P_{t}(A, B)=\int_{A} T_{t} \mathbf{1}_{B} d \mu \text { for } t>0 .
$$

If $\mathrm{d}(A, B)=\infty$, then the situation is simple, with the proof of the following proposition given in Section 5.

Proposition 2.9 Suppose $A, B \in \mathscr{B}_{0}$ satisfy $\mathrm{d}(A, B)=\infty$. Then, under Assumption 2.8, $P_{t}(A, B)=0$ for all $t>0$. In particular, it follows that

$$
\lim _{t \rightarrow 0} t \log P_{t}(A, B)=-\frac{\mathrm{d}(A, B)^{2}}{2}(=-\infty) .
$$

From this proposition, it is sufficient to consider the case when $\mathrm{d}(A, B)<\infty$. For this case, we need some extra assumptions to begin. Let $\log ^{ \pm} x$ denote $0 \vee( \pm \log x)$ for $x \geq 0$.

Theorem 2.10 (Upper Estimate) Let $A, B \in \mathscr{B}_{0}$ with $\mathrm{d}(A, B)<\infty$. Suppose Assumption 2.8 and the following.

(B.1) There exists $\kappa>0$ such that

$$
\left|\int_{E}(b-c, D f)_{H} f d \mu\right| \leq \kappa \mathscr{E}_{1}^{0}(f), \quad f \in \bigcup_{k=1}^{\infty} \mathbb{D}_{E_{k}, b}
$$

(B.2) $A, B$ There exist $\gamma \geq 0$ and nonnegative numbers $\left\{\lambda_{\varepsilon}\right\}_{\varepsilon>0}$ such that

$$
\lim _{\varepsilon \rightarrow 0} \varepsilon \lambda_{\varepsilon}=0
$$

and

$$
\begin{aligned}
& \int_{\left\{0<\mathrm{d}_{B}<\mathrm{d}(A, B)\right\}}\left(b-c, D \mathrm{~d}_{B}\right)_{H} f^{2} d \mu \leq \varepsilon \mathscr{E}^{0}(f)+\lambda_{\varepsilon}+\gamma\left(\int_{E} f^{2} \log ^{+} f^{2} d \mu\right)^{1 / 2} \\
& \text { for any } \varepsilon>0 \text { and } f \in \bigcup_{k=1}^{\infty} \mathbb{D}_{E_{k}, b} \text { with }\|f\|_{2}=1 \text {. }
\end{aligned}
$$

Then,

$$
\varlimsup_{t \rightarrow 0} t \log P_{t}(A, B) \leq-\frac{\mathrm{d}(A, B)^{2}}{2}
$$

Theorem 2.11 (Lower Estimate) Let $B \in \mathscr{B}_{0}$ and $N>0$. Suppose Assumption 2.8 and suppose Eq. 2.8 of Theorem 2.10 holds for any $A \in \mathscr{B}_{0}$ with $\mathrm{d}(A, B)<N$. Then,

$$
\varliminf_{t \rightarrow 0} t \log P_{t}(A, B) \geq-\frac{\mathrm{d}(A, B)^{2}}{2} \text { for any } A \in \mathscr{B}_{0} \text { with } \mathrm{d}(A, B)<N .
$$


Accordingly,

$$
\lim _{t \rightarrow 0} t \log P_{t}(A, B)=-\frac{\mathrm{d}(A, B)^{2}}{2}
$$

for such $A \in \mathscr{B}_{0}$.

In particular, we have the following theorem.

Theorem 2.12 Suppose Assumption 2.8, (B.1) in Theorem 2.10, and the following.

(B.2') There exist $\gamma \geq 0$ and nonnegative numbers $\left\{\lambda_{\varepsilon}\right\}_{\varepsilon>0}$ such that $\lim _{\varepsilon \rightarrow 0} \varepsilon \lambda_{\varepsilon}=0$ and

$$
\int_{E}|b-c|_{H} f^{2} d \mu \leq \varepsilon \mathscr{E}^{0}(f)+\lambda_{\varepsilon}+\gamma\left(\int_{E} f^{2} \log ^{+} f^{2} d \mu\right)^{1 / 2}
$$

for any $\varepsilon>0$ and $f \in \bigcup_{k=1}^{\infty} \mathbb{D}_{E_{k}, b}$ with $\|f\|_{2}=1$.

Then, Eq. 2.10 holds for any $A, B \in \mathscr{B}_{0}$.

Proof Since $\left|D \mathrm{~d}_{B}\right|_{H} \leq 1 \mu$-a.e., (B.2') implies (B.2) ${ }_{A, B}$ for all $A, B \in \mathscr{B}_{0}$ with $\mathrm{d}(A, B)<\infty$. The claim follows from Proposition 2.9, Theorem 2.10, and Theorem 2.11.

Remark 2.13 (i) Assumption 2.8, (B.1), and (B.2') are symmetric with respect to $b$ and $c$, while (B.2) ${ }_{A, B}$ is not.

(ii) The integral in Eq. 2.5 is formally rewritten as $\int_{E}\left(D^{*}(b-c) / 2\right) f^{2} d \mu$, which might be easier to understand.

(iii) We remark that we need only Eq. 2.8, rather than (B.1) and (B.2) $A, B$, for Theorem 2.11 .

A sufficient condition for (B. $\left.2^{\prime}\right)$ is given as follows.

Proposition 2.14 Suppose that $b$ and $c$ are decomposed into $b=b_{1}+b_{2}$ and $c=c_{1}+c_{2}$ such that $b_{1}, b_{2}, c_{1}, c_{2}$ are measurable and the following hold.

(i) There exist nonnegative numbers $\left\{\hat{\lambda}_{\varepsilon}\right\}_{\varepsilon>0}$ such that $\lim _{\varepsilon \rightarrow 0} \varepsilon \hat{\lambda}_{\varepsilon}=0$ and

$$
\int_{E}\left|b_{1}-c_{1}\right|_{H} f^{2} d \mu \leq \varepsilon \mathscr{E}^{0}(f)+\hat{\lambda}_{\varepsilon}\|f\|_{2}^{2}, \quad \varepsilon>0, f \in \bigcup_{k=1}^{\infty} \mathbb{D}_{E_{k}, b} .
$$

(ii) There exists $\delta>0$ such that $\exp \left(\delta\left|b_{2}-c_{2}\right|_{H}^{2}\right)-1 \in L^{1}(\mu)$.

Then, (B. $\left.2^{\prime}\right)$ holds.

The proof is based on a simple application of a type of Hausdorff-Young inequality. We provide the proof in Section 5 together with a discussion of other sufficient conditions. 


\section{Proof of Theorem 2.10}

\section{1 $L^{p}$ Property of Semigroups}

The following proposition is interesting in its own right, as well as being used in the proof of Theorem 2.10. Although claims of the kind made by the proposition have been studied in many papers (e.g., $[5,11,18]$ and the references therein), we give a proof since our conditions seem (at least partially) less restrictive than those in previous studies.

Proposition 3.1 Suppose Assumption 2.8 and the following. There exists $\kappa>0$ such that

$$
\int_{E}(b-c, D f)_{H} f d \mu \leq \kappa \mathscr{E}_{1}^{0}(f), \quad f \in \bigcup_{k=1}^{\infty} \mathbb{D}_{E_{k}, b} .
$$

Then, by letting

$$
q_{0}=\frac{\kappa+2+\sqrt{\kappa^{2}+4(1-\eta)}}{\kappa+\eta}(>2) \quad \text { and } \quad q_{0}^{\prime}=\frac{q_{0}}{q_{0}-1},
$$

$\left\{\left.T_{t}\right|_{L^{2}(\mu) \cap L^{p}(\mu)}\right\}_{t>0}$ (resp., $\left\{\left.\hat{T}_{t}\right|_{L^{2}(\mu) \cap L^{p}(\mu)}\right\}_{t>0}$ ) extends to a strongly continuous semigroup on $L^{p}(\mu)$ for all $p \in\left[2, q_{0}\right]$ (resp., $p \in\left[q_{0}^{\prime}, 2\right]$ ). Moreover, the operator norm of the semigroup on $L^{p}(\mu)$ at $t>0$ is dominated by $\exp \{t(\theta+\kappa|1-2 / p|)\}$.

Proof It suffices to consider $\left\{T_{t}\right\}_{t>0}$; the claim for $\left\{\hat{T}_{t}\right\}_{t>0}$ follows by considering the adjoint semigroup of $\left\{T_{t}\right\}_{t>0}$.

Let $p \geq 2$ and $M \geq 1$. Define a $C^{2}$-function $\Lambda$ on $[0, \infty)$ so that $\Lambda(0)=\Lambda^{\prime}(0)=0$ and $\Lambda^{\prime \prime}(x)=p(p-1)(x \wedge M)^{p-2}$ for $x \geq 0$. Also, define the following functions on $[0, \infty)$ :

$$
\hat{\Lambda}(x)=\int_{0}^{x} \sqrt{\Lambda^{\prime \prime}(s)} d s, \quad \tilde{\Lambda}(x)=\sqrt{\Lambda^{\prime}(x) x}, \quad \text { and } \quad \check{\Lambda}(x)=\sqrt{\Lambda^{\prime}(x) x-2 \Lambda(x)} .
$$

Then, by long but straightforward calculation, we can confirm the following inequalities:

$$
\begin{aligned}
& \Lambda^{\prime}(x) \geq \frac{p}{2(p-1)} \hat{\Lambda}(x) \hat{\Lambda}^{\prime}(x), \quad 0 \leq \tilde{\Lambda}^{\prime}(x) \leq \frac{p}{2 \sqrt{p-1}} \hat{\Lambda}^{\prime}(x), \\
& \text { and } 0 \leq \check{\Lambda}^{\prime}(x) \leq \sqrt{\frac{p(p-2)}{4(p-1)}} \hat{\Lambda}^{\prime}(x) .
\end{aligned}
$$

Since $\hat{\Lambda}(0)=\tilde{\Lambda}(0)=\check{\Lambda}(0)=0$, this implies, in particular,

$$
\begin{aligned}
& \Lambda(x) \geq \frac{p}{4(p-1)} \hat{\Lambda}(x)^{2}, \quad 0 \leq \tilde{\Lambda}(x) \leq \frac{p}{2 \sqrt{p-1}} \hat{\Lambda}(x), \\
& \text { and } \quad 0 \leq \check{\Lambda}(x) \leq \sqrt{\frac{p(p-2)}{4(p-1)}} \hat{\Lambda}(x) .
\end{aligned}
$$

Let $C \geq 0$ and $t>0$. Take $f \in L^{2}(\mu) \cap L^{p}(\mu)$ with $f \geq 0 \mu$-a.e. Since $T_{t} f \geq 0 \mu$-a.e. and $\Lambda^{\prime \prime}$ is bounded, we have that $\Lambda^{\prime}\left(T_{t} f\right) \in L^{2}(\mu), \Lambda\left(T_{t} f\right) \in L^{1}(\mu)$ and

$$
\begin{aligned}
\frac{d}{d t} \int_{E} e^{-C t} \Lambda\left(T_{t} f\right) d \mu & =\int_{E}\left(-C e^{-C t} \Lambda\left(T_{t} f\right)+e^{-C t} \Lambda^{\prime}\left(T_{t} f\right) \mathscr{L} T_{t} f\right) d \mu \\
& =e^{-C t}\left(-C \int_{E} \Lambda\left(T_{t} f\right) d \mu-\mathscr{E}\left(T_{t} f, \Lambda^{\prime}\left(T_{t} f\right)\right)\right)
\end{aligned}
$$


Moreover, for $v \in \bigcup_{k=1}^{\infty} \mathbb{D}_{E_{k}, b,+}$,

$$
\begin{aligned}
\mathscr{E}\left(v, \Lambda^{\prime}(v)\right)= & \frac{1}{2} \int_{E}\left(D v, D\left(\Lambda^{\prime}(v)\right)\right)_{H} d \mu+\int_{E}(b, D v)_{H} \Lambda^{\prime}(v) d \mu \\
& +\int_{E}\left(c, D\left(\Lambda^{\prime}(v)\right)\right)_{H} v d \mu+\int_{E} V v \Lambda^{\prime}(v) d \mu \\
= & \frac{1}{2} \int_{E}|D(\hat{\Lambda}(v))|_{H}^{2}+\int_{E}(b+c, D(\tilde{\Lambda}(v)))_{H} \tilde{\Lambda}(v) d \mu \\
& -\int_{E}(b-c, D(\check{\Lambda}(v)))_{H} \check{\Lambda}(v) d \mu+\int_{E} V \tilde{\Lambda}(v)^{2} d \mu \\
\geq & \mathscr{E}^{0}(\hat{\Lambda}(v))-\eta \mathscr{E}^{0}(\tilde{\Lambda}(v))-\theta\|\tilde{\Lambda}(v)\|_{2}^{2}-\kappa_{1}^{0}(\check{\Lambda}(v)) . \quad \text { (from (A.2) and (3.1)) }
\end{aligned}
$$

By using Eqs. 3.3, 3.4, and the derivation property (2.1) of $D$, we have

$$
\begin{aligned}
& -C \int_{E} \Lambda(v) d \mu-\mathscr{E}\left(v, \Lambda^{\prime}(v)\right) \\
& \leq-\frac{C p}{4(p-1)}\|\hat{\Lambda}(v)\|_{2}^{2}-\mathscr{E}^{0}(\hat{\Lambda}(v))+\frac{\eta p^{2}}{4(p-1)} \mathscr{E}^{0}(\hat{\Lambda}(v)) \\
& \quad+\frac{\theta p^{2}}{4(p-1)}\|\hat{\Lambda}(v)\|_{2}^{2}+\frac{\kappa p(p-2)}{4(p-1)} \mathscr{E}_{1}^{0}(\hat{\Lambda}(v)) \\
& =\frac{-4(p-1)+\eta p^{2}+\kappa p(p-2)}{4(p-1)} \mathscr{E}^{0}(\hat{\Lambda}(v))+\frac{p\{-C+\theta p+\kappa(p-2)\}}{4(p-1)}\|\hat{\Lambda}(v)\|_{2}^{2} .
\end{aligned}
$$

Therefore, if $-4(p-1)+\eta p^{2}+\kappa p(p-2) \leq 0$ - or, more specifically, if $p \in\left[2, q_{0}\right]$ with $q_{0}$ given by Eq. 3.2-then the right-hand side of Eq. 3.5 is non-positive by letting $C=C_{p}:=\theta p+\kappa(p-2)$. Thus, the inequality

$$
-C_{p} \int_{E} \Lambda(v) d \mu-\mathscr{E}\left(v, \Lambda^{\prime}(v)\right) \leq 0
$$

is valid for all $v \in \mathbb{D}$ with $v \geq 0 \mu$-a.e., by approximating $v$ by elements of $\bigcup_{k=1}^{\infty} \mathbb{D}_{E_{k}, b,+}$. In particular,

$$
\frac{d}{d t} \int_{E} e^{-C_{p} t} \Lambda\left(T_{t} f\right) d \mu \leq 0,
$$

which implies

$$
\int_{E} e^{-C_{p} t} \Lambda\left(T_{t} f\right) d \mu \leq \int_{E} \Lambda(f) d \mu, \quad t>0 .
$$

Letting $M \rightarrow \infty$, we obtain that $\int_{E} e^{-C_{p} t}\left(T_{t} f\right)^{p} d \mu \leq \int_{E} f^{p} d \mu$, from the monotone convergence theorem. Thus, $\left\{\left.T_{t}\right|_{L^{2}(\mu) \cap L^{p}(\mu)}\right\}_{t>0}$ extends to a semigroup on $L^{p}(\mu)$ that satisfies $\left\|T_{t}\right\|_{L^{p}(\mu) \rightarrow L^{p}(\mu)} \leq e^{C_{p} t / p}$. The strong continuity of the semigroup follows from the result given in [20]. (Indeed, it is easy to see that $\left\{T_{t}\right\}_{t>0}$ is a weakly continuous semigroup on $L^{p}(\mu)$, which implies strong continuity.)

Corollary 3.2 Suppose Assumption 2.8 and (B.1). Then, the operators $\left\{\left.T_{t}\right|_{L^{2}(\mu) \cap L^{p}(\mu)}\right\}_{t>0}$ and $\left\{\left.\hat{T}_{t}\right|_{L^{2}(\mu) \cap L^{p}(\mu)}\right\}_{t>0}$ extend to strongly continuous semigroups on $L^{p}(\mu)$ for all $p \in$ $\left[q_{0}^{\prime}, q_{0}\right]$, where $q_{0}$ and $q_{0}^{\prime}$ are as given in Eq. 3.2. Moreover, the operator norm of the semigroups on $L^{p}(\mu)$ at $t>0$ are dominated by $\exp \{t(\theta+\kappa|1-2 / p|)\}$.

Proof Apply Proposition 3.1 to $(\mathscr{E}, \mathbb{D})$ and $(\hat{\mathscr{E}}, \mathbb{D})$. 


\subsection{Preliminary Estimates}

In this subsection, we provide several quantitative estimates used in the proof of Theorem 2.10.

We take a non-decreasing $C^{2}$-function $\xi$ on $[0, \infty)$ such that $\xi(x)=(x-1)^{3} \vee 0$ for $x \in[0,3 / 2]$ and $\xi(x)=1$ for $x \in[2, \infty)$. Define

$$
\zeta(x, y)=2 x+\left(y x^{y-1}-2 x\right) \xi(x), \quad x \geq 0, y \geq 2,
$$

and

$$
\tau(x, y)=\int_{0}^{x} \zeta(s, y) d s, \quad x \geq 0, y \geq 2
$$

Then,

$$
\partial_{x} \zeta(x, y)=2+\left(y(y-1) x^{y-2}-2\right) \xi(x)+\left(y x^{y-1}-2 x\right) \xi^{\prime}(x) \geq 2 .
$$

For $R>2$, we define the following functions on $[0, \infty) \times[2, \infty)$ :

$$
\begin{aligned}
g_{R}(x, y) & =\sqrt{\left(\partial_{x} \zeta\right)(x \wedge R, y)}, & & h_{R}(x, y)=\int_{0}^{x} g_{R}(s, y)^{2} d s, \\
\phi_{R}(x, y) & =\int_{0}^{x} h_{R}(s, y) d s, & \rho_{R}(x, y) & =\int_{0}^{x} g_{R}(s, y) d s, \\
\iota_{R}(x, y) & =x h_{R}(x, y)-2 \phi_{R}(x, y), & \psi_{R}(x, y) & =\sqrt{x h_{R}(x, y)} .
\end{aligned}
$$

Lemma 3.3 For any fixed $y \geq 2$, the following hold.

(i) For $x \geq 0$,

$$
\begin{aligned}
h_{R}(x, y) & =\zeta(x \wedge R, y)+((x-R) \vee 0) \partial_{x} \zeta(R, y) \\
& =\zeta(x \wedge R, y)+((x-R) \vee 0) y(y-1) R^{y-2} .
\end{aligned}
$$

In particular, $h_{R}(x, y)=\zeta(x, y)$ if $0 \leq x \leq R$.

(ii) $2 x \leq h_{R}(x, y) \leq \zeta(x, y) \leq \max \left\{2 x, y x^{y-1}\right\}$ for all $R>2$ and $x \geq 0$.

(iii) For $x \geq 0, h_{R}(x, y)$ converges to $\zeta(x, y)$ as $R \rightarrow \infty$.

(iv) $x^{2} \leq \phi_{R}(x, y) \leq \tau(x, y) \leq \max \left\{x^{2}, x^{y}\right\}$ for all $R>2$ and $x \geq 0$.

(v) $g_{R}(x, y) \geq \sqrt{2}$ for $x \geq 0$ and $g_{R}(x, y) \geq \sqrt{y(y-1)}(x \wedge R)^{y / 2-1}$ for $x \geq 2$.

Proof (i) and (v): Straightforward from the definitions.

(ii): The first and last inequalities are easy to prove. Since

$$
\partial_{x} h_{R}(x, y)=\partial_{x} \zeta(x \wedge R, y) \leq \partial_{x} \zeta(x, y),
$$

the second inequality also holds.

(iii): This follows from (i).

(iv): This follows by integrating each term of the inequality in (ii).

Lemma 3.4 For any $x \geq 0$ and $y \geq 2$, the following hold.

(i) $x g_{R}(x, y)^{2} \geq h_{R}(x, y)$.

(ii) $\iota_{R}(x, y) \geq 0$.

(iii) $\rho_{R}(x, y) \leq x g_{R}(x, y)$. 
Proof (i): For $x \in[0, R]$,

$$
\begin{aligned}
x g_{R}(x, y)^{2}-h_{R}(x, y) & =x \partial_{x} \zeta(x, y)-\zeta(x, y) \\
& =y(y-2) x^{y-1} \xi(x)+\left(y x^{y}-2 x^{2}\right) \xi^{\prime}(x),
\end{aligned}
$$

which is nonnegative since $\xi^{\prime}(x)=0$ for $x \leq 1$. For $x>R$,

$$
\begin{aligned}
x g_{R}(x, y)^{2}-h_{R}(x, y) & =x \partial_{x} \zeta(R, y)-\left\{\zeta(R, y)-(x-R) \partial_{x} \zeta(R, y)\right\} \\
& =y(y-2) R^{y-1} \geq 0 .
\end{aligned}
$$

(ii): This follows from identities $\iota_{R}(0, y)=0, \partial_{x} \iota_{R}(x, y)=x g_{R}(x, y)^{2}-h_{R}(x, y)$, and (i).

(iii): By the Cauchy-Schwarz inequality,

$$
\begin{aligned}
\rho_{R}(x, y) & =\int_{0}^{x} g_{R}(s, y) d s \leq\left(x \int_{0}^{x} g_{R}(s, y)^{2} d s\right)^{1 / 2} \\
& =\left(x h_{R}(x, y)\right)^{1 / 2} \leq x g_{R}(x, y) . \quad(\text { from (i) })
\end{aligned}
$$

Lemma 3.5 For $y \in[2,3], \sqrt{\iota_{R}(x, y)}$ is continuously differentiable with respect to $x$. Moreover, there exists some $K_{0}>0$ independent of $x$ and $y$ such that

$$
0 \leq \partial_{x} \sqrt{\iota_{R}(x, y)} \leq K_{0} \sqrt{y-2} g_{R}(x, y)
$$

and

$$
0 \leq \sqrt{\iota_{R}(x, y)} \leq K_{0} \sqrt{y-2} \rho_{R}(x, y)
$$

Proof Since $\iota_{R}(x, 2) \equiv 0$, it suffices to consider the case $y \in(2,3]$. The continuous differentiability of $\sqrt{\iota_{R}(x, y)}$ is trivial for $x \neq 1$ since $\iota_{R}(x, y)=0$ for $x \in[0,1]$ and $\iota_{R}(x, y)>0$ for $x>1$. For $x \in(1, R]$, combining Eqs. 3.7 and 3.8,

$$
\partial_{x} \iota_{R}(x, y)=\left\{\begin{array}{l}
(y-2)\left[y x^{y-1} \xi(x)+\left\{x^{y}+\frac{2\left(x^{y}-x^{2}\right)}{y-2}\right\} \xi^{\prime}(x)\right] \\
y(y-2) R^{y-1} \\
(1<x \leq R), \\
(x>R) .
\end{array}\right.
$$

Thus, we can confirm that there exist $K_{1}$ and $K_{2}$ with $0<K_{1}<K_{2}$ such that

$$
K_{1}(y-2) \hat{\imath}(x, y) \leq \partial_{x} \iota_{R}(x, y) \leq K_{2}(y-2) \hat{\imath}(x, y) \text { for } x \in(1, R] \text { and } y \in(2,3],
$$

where $\hat{\imath}$ is defined as

$$
\hat{\imath}(x, y)=(x-1)^{2} \mathbf{1}_{(1,3 / 2]}(x)+\mathbf{1}_{(3 / 2,2]}(x)+x^{y-1} \mathbf{1}_{(2, R]}(x) .
$$

For $x \in(1,3 / 2]$, we have

$$
K_{1}(x-1)^{3}(y-2) / 3 \leq \iota_{R}(x, y) \leq K_{2}(x-1)^{3}(y-2) / 3 .
$$

Thus, $\lim _{x \downarrow 1} \sqrt{\iota_{R}(x, y)} /(x-1)=0$. That is, $\sqrt{\iota_{R}(x, y)}$ is continuously differentiable with respect to $x$ at 1 . Furthermore,

$$
0<\frac{\partial_{x} \iota_{R}(x, y)}{\sqrt{\iota_{R}(x, y)}} \leq K_{2} \sqrt{3 / K_{1}} \sqrt{x-1} \sqrt{y-2} \leq K_{2} K_{1}^{-1 / 2} \sqrt{y-2} g_{R}(x, y)
$$

from Lemma 3.3(v). 
For $x \in(3 / 2,2]$, we have $\iota_{R}(x, y) \geq \iota_{R}(3 / 2, y) \geq K_{1}(y-2) / 24$ and

$$
0<\frac{\partial_{x} \iota_{R}(x, y)}{\sqrt{\iota_{R}(x, y)}} \leq K_{2} \sqrt{24 / K_{1}} \sqrt{y-2} \leq K_{2} \sqrt{12 / K_{1}} \sqrt{y-2} g_{R}(x, y) .
$$

For $x \in(2, R]$,

$$
\begin{aligned}
\iota_{R}(x, y) & \geq \iota_{R}(2, y)+\int_{2}^{R} \partial_{x} \iota_{R}(s, y) d s \geq \frac{K_{1}}{24}(y-2)+K_{1}(y-2) \frac{x^{y}-2^{y}}{y} \\
& \geq \frac{K_{1}}{24}(y-2)\left(1+\frac{x^{y}-2^{y}}{8}\right) \geq K_{3}(y-2) x^{y}
\end{aligned}
$$

for $K_{3}=K_{1} / 192$, and

$$
0<\frac{\partial_{x} \iota_{R}(x, y)}{\sqrt{\iota_{R}(x, y)}} \leq K_{2} K_{3}^{-1 / 2} \sqrt{y-2} x^{y / 2-1} \leq K_{2} K_{3}^{-1 / 2} \sqrt{y-2} g_{R}(x, y) .
$$

For $x>R$, we have $\iota_{R}(x, y) \geq \iota_{R}(R, y) \geq K_{3}(y-2) R^{y}$ and

$$
0<\frac{\partial_{x} \iota_{R}(x, y)}{\sqrt{\iota_{R}(x, y)}} \leq K_{3}^{-1 / 2} \sqrt{y-2} R^{y / 2-1} \leq \sqrt{2 / K_{3}} \sqrt{y-2} g_{R}(x, y) .
$$

From these estimates, Eq. 3.9 holds by setting

$$
K_{0}=2^{-1} \max \left\{K_{2} \sqrt{12 / K_{1}}, K_{2} K_{3}^{-1 / 2}, \sqrt{2 / K_{3}}\right\} .
$$

Integrating each term of Eq. 3.9 gives Eq. 3.10.

Lemma 3.6 For $x \geq 0$ and $y \in[2,3]$,

$$
\max \left\{0, x^{y} \log ^{+} x-(2 \log 2) x^{2}\right\} \leq \partial_{y} \tau(x, y) \leq x^{y} \log ^{+} x .
$$

Proof We have

$$
\partial_{y} \tau(x, y)=\int_{0}^{x} \partial_{y} \zeta(s, y) d s=\int_{0}^{x}\left(s^{y-1}+y s^{y-1} \log s\right) \xi(s) d s .
$$

From $\xi(x)=0$ for $x \in[0,1]$, it follows that $\partial_{y} \tau(x, y)$ vanishes for $x \in[0,1]$ and is non-decreasing in $x$. For $x \in[0,2]$ and $y \in[2,3]$,

$$
x^{y} \log ^{+} x-(2 \log 2) x^{2} \leq x^{2}\left(2^{y-2} \log 2-2 \log 2\right) \leq 0 \leq \partial_{y} \tau(x, y) .
$$

Moreover, for $x \geq 2$ and $y \in[2,3]$,

$$
\begin{aligned}
\partial_{y} \tau(x, y) & \geq \int_{2}^{x}\left(s^{y-1}+y s^{y-1} \log s\right) d s \\
& =\left.s^{y} \log s\right|_{s=2} ^{s=x} \\
& =x^{y} \log x-2^{y} \log 2 \\
& \geq x^{y} \log ^{+} x-(2 \log 2) x^{2} .
\end{aligned}
$$

Therefore, the first inequality of Eq. 3.11 holds. For $x>1$, we have

$$
\partial_{y} \tau(x, y) \leq \int_{1}^{x}\left(s^{y-1}+y s^{y-1} \log s\right) d s=\left.s^{y} \log s\right|_{s=1} ^{s=x}=x^{y} \log x .
$$

Thus, the second inequality of Eq. 3.11 holds.

Lemma 3.7 For each $\varepsilon>0$, there exists some $y_{0}=y_{0}(\varepsilon)>2$ such that $g_{R}(x, y) x^{-\varepsilon}$ is non-increasing in $x$ for any $y \in\left[2, y_{0}\right]$ and $R>2$. 
Proof Since $g_{R}(x, y)=g_{R}(R, y)$ for $x \geq R$, the term $g_{R}(x, y) x^{-\varepsilon}$ is always nonincreasing for $x \geq R$. It therefore suffices to consider only $x$ in $[0, R]$. We may additionally assume that $\varepsilon \in(0,1 / 2)$. Define

$$
v(x, y):=\left(g_{R}(x, y) x^{-\varepsilon}\right)^{2}, \quad(x, y) \in[0, R] \times[2, \infty) .
$$

It suffices to prove that there exists some $y_{0}(\varepsilon)>2$ such that, for $(x, y) \in[0, R] \times$ $\left[2, y_{0}(\varepsilon)\right]$,

$$
\partial_{x} v(x, y) \leq 0 .
$$

By definition, for $(x, y) \in[0, R] \times[2, \infty)$,

$$
\begin{aligned}
v(x, y) & =\partial_{x} \zeta(x, y) x^{-2 \varepsilon} \\
& =2 x^{-2 \varepsilon}+\left(y(y-1) x^{y-2-2 \varepsilon}-2 x^{-2 \varepsilon}\right) \xi(x)+\left(y x^{y-1-2 \varepsilon}-2 x^{1-2 \varepsilon}\right) \xi^{\prime}(x)
\end{aligned}
$$

and

$$
\begin{aligned}
\partial_{x} v(x, y)= & -4 \varepsilon x^{-1-2 \varepsilon}+\left(y(y-1)(y-2-2 \varepsilon) x^{y-3-2 \varepsilon}+4 \varepsilon x^{-1-2 \varepsilon}\right) \xi(x) \\
& +\left(2 y(y-1-\varepsilon) x^{y-2-2 \varepsilon}-4(1-\varepsilon) x^{-2 \varepsilon}\right) \xi^{\prime}(x)+\left(y x^{y-1-2 \varepsilon}-2 x^{1-2 \varepsilon}\right) \xi^{\prime \prime}(x) .
\end{aligned}
$$

Suppose $0 \leq x \leq 1$. Since $\xi(x)=\xi^{\prime}(x)=\xi^{\prime \prime}(x)=0$,

$$
\partial_{x} v(x, y)=-4 \varepsilon x^{-1-2 \varepsilon}
$$

thus, Eq. 3.12 holds for this case.

Suppose $2 \leq x \leq R$. Since $\xi(x)=1$ and $\xi^{\prime}(x)=\xi^{\prime \prime}(x)=0$,

$$
\partial_{x} v(x, y)=y(y-1)(y-2-2 \varepsilon) x^{y-3-2 \varepsilon} .
$$

Therefore, Eq. 3.12 holds for $y \in[2,2+2 \varepsilon]$.

Since $\xi, \xi^{\prime}$, and $\xi^{\prime \prime}$ are all bounded, $\partial_{x} v(x, y)$ converges to $-4 \varepsilon x^{-1-2 \varepsilon}$ uniformly in $x \in[1,2]$ as $y \rightarrow 2$. Thus, there exists some $y_{0}(\varepsilon) \in(2,2+2 \varepsilon]$ such that

$$
\left|\partial_{x} v(x, y)+4 \varepsilon x^{-1-2 \varepsilon}\right| \leq 4 \varepsilon \cdot 2^{-1-2 \varepsilon}, \quad(x, y) \in[1,2] \times\left[2, y_{0}(\varepsilon)\right] .
$$

Equation 3.13 implies Eq. 3.12 for $(x, y) \in[1,2] \times\left[2, y_{0}(\varepsilon)\right]$.

Lemma 3.8 The following inequalities hold for $\delta \in(0,1 / 2), R>2, x \geq 0$, and $y \in$ $\left[2, y_{0}(\delta)\right]:$

(i) $x g_{R}(x, y) \leq(1+\delta) \rho_{R}(x, y)$,

(ii) $x g_{R}(x, y)^{2} \leq(1+2 \delta) h_{R}(x, y)$,

(iii) $x h_{R}(x, y) \leq(1+\delta)^{2} \rho_{R}(x, y)^{2}$,

(iv) $x g_{R}(x, y)^{2}-h_{R}(x, y) \leq \frac{2 \delta(1+\delta)}{1+2 \delta} \rho_{R}(x, y) g_{R}(x, y)$,

(v) $\rho_{R}(x, y)^{2} \leq 2(1+2 \delta) \phi_{R}(x, y) \leq 2(1+2 \delta) \tau(x, y)$,

(vi) $\left(\partial_{x} \psi_{R}(x, y)\right)^{2} \leq(1+2 \delta) g_{R}(x, y)^{2}$.

Proof In the following, we omit $y$ from the notation.

From Lemma 3.7, $g_{R}(s) s^{-\delta} \geq g_{R}(x) x^{-\delta} \geq 0$ if $0<s \leq x$. Then, we have

$$
\rho_{R}(x)=\int_{0}^{x} g_{R}(s) d s \geq g_{R}(x) x^{-\delta} \int_{0}^{x} s^{\delta} d s=\frac{x g_{R}(x)}{1+\delta}
$$


and

$$
h_{R}(x)=\int_{0}^{x} g_{R}(s)^{2} d s \geq g_{R}(x)^{2} x^{-2 \delta} \int_{0}^{x} s^{2 \delta} d s=\frac{x g_{R}(x)^{2}}{1+2 \delta} .
$$

Thus, (i) and (ii) hold. Combining (i) and Lemma 3.4(i) gives (iii). From (i) and (ii),

$$
x g_{R}(x)^{2}-h_{R}(x) \leq\left(1-\frac{1}{1+2 \delta}\right) x g_{R}(x)^{2} \leq \frac{2 \delta(1+\delta)}{1+2 \delta} \rho_{R}(x) g_{R}(x),
$$

which proves (iv). Next, we prove (v). From (ii) and Lemma 3.4(iii), we have

$$
\partial_{x}\left(\rho_{R}(x)^{2}\right)=2 g_{R}(x) \rho_{R}(x) \leq 2 x g_{R}(x)^{2} \leq 2(1+2 \delta) h_{R}(x) .
$$

Then,

$$
\rho_{R}(x)^{2} \leq 2(1+2 \delta) \int_{0}^{x} h_{R}(s) d s=2(1+2 \delta) \phi_{R}(x) .
$$

The second inequality of (v) follows from Lemma 3.3(iv).

Last, we prove (vi). The inequality holds for $x=0$ by direct computation. Let $x>0$. By using (ii) and Lemma 3.4(i), we have

$$
\left(\partial_{x} \psi_{R}(x)\right)^{2}=\frac{\left(x g_{R}(x)^{2}+h_{R}(x)\right)^{2}}{4 x h_{R}(x)} \leq \frac{\left(2 x g_{R}(x)^{2}\right)^{2}}{4(1+2 \delta)^{-1} x^{2} g_{R}(x)^{2}}=(1+2 \delta) g_{R}(x)^{2} .
$$

\subsection{Derivation of a Differential Inequality}

In this and further subsections, we prove Theorem 2.10. The following inequality is often used throughout this paper without specific mention:

$$
|x y| \leq \frac{1}{\alpha} x^{2}+\frac{\alpha}{4} y^{2} \quad \text { for } \alpha>0 \text { and } x, y \in \mathbb{R} .
$$

Let $\varepsilon \in(0,(1-\eta) / 6]$. Take $\delta>0$ such that

$$
(1+\delta)^{2} \leq 1+\varepsilon
$$

In particular, we use $2 \delta \leq \varepsilon \leq 1 / 6$. Let

$$
q=\min \left\{3,1+\frac{q_{0}}{2}, y(\delta), 2+\frac{\varepsilon}{\kappa K_{0}^{2}}\right\} \in(2,3] \quad \text { and } \quad S=\frac{3 \gamma^{2}}{\varepsilon} .
$$

Here, $q_{0}, y(\cdot)$, and $K_{0}$ are as provided in Eq. 3.2, Lemma 3.7, and Lemma 3.5, respectively. We set

$$
t_{0}=\min \{1,(q-2) / S\} \quad \text { and } \quad p(t)=q-S t, \quad t \in\left[0, t_{0}\right] .
$$

Note that $p(t) \in[2, q]$ for $t \in\left[0, t_{0}\right]$.

Let $A, B \in \mathscr{B}_{0}$ with $\mathrm{d}(A, B)<\infty$ and set $w=\mathrm{d}_{B} \wedge \mathrm{d}(A, B) \in \mathbb{D}_{0}$. Let $\alpha \in \mathbb{R} \backslash\{0\}$ and define

$$
u_{t}=T_{t} \mathbf{1}_{B}, F(t)=e^{\alpha w} u_{t}, \text { and } \sigma(t)=\int_{E} \tau(F(t), p(t)) d \mu \quad \text { for } t \in\left[0, t_{0}\right] .
$$


Lemma 3.9 The function $\sigma$ is continuously differentiable on $\left(0, t_{0}\right]$ and

$$
\begin{aligned}
\sigma^{\prime}(t) & =\int_{E}\left\{\partial_{x} \tau(F(t), p(t)) F^{\prime}(t)+\partial_{y} \tau(F(t), p(t)) p^{\prime}(t)\right\} d \mu \\
& =\int_{E} \zeta(F(t), p(t)) e^{\alpha w} \mathscr{L} u_{t} d \mu-S \int_{E} \partial_{y} \tau(F(t), p(t)) d \mu .
\end{aligned}
$$

Proof We justify the formal calculation. First, let us recall the following fact (see, e.g., [2, Theorems 21.4 and 21.8]): Suppose $r \in[1, \infty)$ and that $\chi \in L^{1}(\mu)$ satisfies $0<\chi \leq 1$ $\mu$-a.e. Then, for functions $\left\{f_{n}\right\}_{n=1}^{\infty}$ in $L^{r}(\mu)$ and $f \in L^{0}(\mu)$, the sequence $f_{n}$ converges to $f$ in $L^{r}(\mu)$ if and only if $f_{n}$ converges to $f$ in measure with respect to $\chi \cdot \mu$ and

$$
\lim _{K \rightarrow \infty} \sup _{n} \int_{E}\left\{\left(\left|f_{n}\right|^{r}-K \chi\right) \vee 0\right\} d \mu=0 .
$$

For $t \in\left(0, t_{0}\right]$ and $\left\{t_{n}\right\}_{n=1}^{\infty} \subset\left(0, t_{0}\right] \backslash\{t\}$ converging to $t$,

$$
\begin{aligned}
& \left(\tau\left(F\left(t_{n}\right), p\left(t_{n}\right)\right)-\tau(F(t), p(t))\right) /\left(t_{n}-t\right) \\
= & \tau\left(F(t)+s\left(F\left(t_{n}\right)-F(t)\right), p(t)+s\left(p\left(t_{n}\right)-p(t)\right)\right) \mid \begin{array}{l}
s=1 \\
s=0
\end{array} /\left(t_{n}-t\right) \\
= & \left(t_{n}-t\right)^{-1} \int_{0}^{1}\left\{\left(F\left(t_{n}\right)-F(t)\right) \partial_{x} \tau\left(g_{s, n}, h_{s, n}\right)+\left(p\left(t_{n}\right)-p(t)\right) \partial_{y} \tau\left(g_{s, n}, h_{s, n}\right)\right\} d s \\
= & \frac{F\left(t_{n}\right)-F(t)}{t_{n}-t} \int_{0}^{1} \zeta\left(g_{s, n}, h_{s, n}\right) d s-S \int_{0}^{1} \partial_{y} \tau\left(g_{s, n}, h_{s, n}\right) d s,
\end{aligned}
$$

where $g_{s, n}=F(t)+s\left(F\left(t_{n}\right)-F(t)\right)(\geq 0)$ and $h_{s, n}=p(t)+s\left(p\left(t_{n}\right)-p(t)\right)$. For each $s \in[0,1], g_{s, n}$ converges to $F(t)$ in $L^{r}$ as $n \rightarrow \infty$ for every $r \in\left[2, q_{0}\right]$, and $h_{s, n}$ converges to $p(t)$ as $n \rightarrow \infty$. In particular, for every $s \in[0,1]$ and $r \in\left[2, q_{0}\right], g_{s, n}$ converges to $F(t)$ in measure with respect to $\chi \cdot \mu$ and

$$
\lim _{K \rightarrow \infty} \sup _{n} \int_{E}\left\{\left(\left(g_{s, n}\right)^{r}-K \chi\right) \vee 0\right\} d \mu=0 .
$$

From the continuity of $\zeta, \zeta\left(g_{s, n}, h_{s, n}\right)$ converges to $\zeta(F(t), p(t))$ in measure with respect to $\chi \cdot \mu$ as $n \rightarrow \infty$. Lemma 3.3(ii) and the inequality $2<2(q-1)<q_{0}$ together imply $\zeta(x, y)^{2} \leq 4 x^{2}+q_{0}^{2} x^{q_{0}}$ for $x \geq 0$ and $y \in\left[2, q_{0}\right]$. Thus,

$$
\begin{aligned}
& \sup _{n} \int_{E}\left\{\left(\zeta\left(g_{s, n}, h_{s, n}\right)^{2}-K \chi\right) \vee 0\right\} d \mu \\
\leq & \sup _{n} \int_{E}\left\{\left(4\left(g_{s, n}\right)^{2}-K \chi / 2\right) \vee 0\right\} d \mu+\sup _{n} \int_{E}\left\{\left(q_{0}^{2}\left(g_{s, n}\right)^{q_{0}}-K \chi / 2\right) \vee 0\right\} d \mu \\
\rightarrow & 0 \quad \text { as } K \rightarrow \infty .
\end{aligned}
$$

From the above, $\zeta\left(g_{s, n}, h_{s, n}\right)$ converges to $\zeta(F(t), p(t))$ in $L^{2}(\mu)$. Moreover, since it is easy to see that $\left\{\zeta\left(g_{s, n}, h_{s, n}\right)\right\}_{s \in[0,1], n \in \mathbb{N}}$ is bounded in $L^{2}(\mu)$, we obtain

$$
\left\|\int_{0}^{1} \zeta\left(g_{s, n}, h_{s, n}\right) d s-\zeta(F(t), p(t))\right\|_{2} \leq \int_{0}^{1}\left\|\zeta\left(g_{s, n}, h_{s, n}\right)-\zeta(F(t), p(t))\right\|_{2} d s \rightarrow 0
$$

as $n \rightarrow \infty$, by the dominated convergence theorem. Thus, the first term of Eq. 3.17 converges to $F^{\prime}(t) \zeta(F(t), p(t))$ in $L^{1}(\mu)$ as $n \rightarrow \infty$ because $\left(F\left(t_{n}\right)-F(t)\right) /\left(t_{n}-t\right)$ converges to $F^{\prime}(t)$ in $L^{2}(\mu)$. 
In the same manner, we can prove that $\int_{0}^{1} \partial_{y} \tau\left(g_{s, n}, h_{s, n}\right) d s$ converges to $\partial_{y} \tau(F(t), p(t))$ in $L^{1}(\mu)$ as $n \rightarrow \infty$, by using Lemma 3.6. Thus, Eq. 3.16 is proved. The proof of the continuity of $\sigma^{\prime}(t)$ proceeds analogously.

We fix $t \in\left(0, t_{0}\right]$ and estimate the first term of Eq. 3.16. Recall the measurable nest $\left\{E_{k}\right\}_{k=1}^{\infty}$ in Assumption 2.8. Take a sequence of functions $\left\{w_{k}\right\}_{k=1}^{\infty}$ such that for every $k \in \mathbb{N}$, $w_{k} \in \mathbb{D}_{E_{k}, b}, w_{k}=w \mu$-a.e. on $E_{k}$ and $0 \leq w_{k} \leq N \mu$-a.e. There also exist functions $\left\{u^{(k)}\right\}_{k=1}^{\infty}$ such that $u^{(k)} \in \mathbb{D}_{E_{k}, b}$ for each $k$ and the sequence $u^{(k)}$ converges to $u_{t}$ in $\mathbb{D}$ as $k \rightarrow \infty$. By considering $0 \vee\left(u^{(k)} \wedge u_{t}\right)$ (and the Cesàro means if necessary) we may assume that $0 \leq u^{(k)} \leq u_{t} \mu$-a.e. for every $k$ and $\lim _{k \rightarrow \infty} u^{(k)}=u_{t} \mu$-a.e. Let $F^{(k)}=e^{\alpha w_{k}} u^{(k)} \in$ $\mathbb{D}_{E_{k}, b}$ for each $k$. Then,

$$
0 \leq F^{(k)}=e^{\alpha w} u^{(k)} \leq F(t) \quad \mu \text {-a.e. }
$$

and

$$
\begin{aligned}
\int_{E} \zeta(F(t), p(t)) e^{\alpha w} \mathscr{L} u_{t} d \mu & =\int_{E} \lim _{R \rightarrow \infty} h_{R}(F(t), p(t)) e^{\alpha w} \mathscr{L} u_{t} d \mu \quad \text { (from Lemma 3.3) } \\
& =\lim _{R \rightarrow \infty} \int_{E} \lim _{k \rightarrow \infty} h_{R}\left(F^{(k)}, p(t)\right) e^{\alpha w_{k}} \mathscr{L} u_{t} d \mu \\
& =\lim _{R \rightarrow \infty} \lim _{k \rightarrow \infty}-\mathscr{E}\left(u_{t}, h_{R}\left(F^{(k)}, p(t)\right) e^{\alpha w_{k}}\right) .
\end{aligned}
$$

Lemma 3.10 For any $R>2$,

$$
\lim _{k \rightarrow \infty} \mathscr{E}\left(u_{t}, h_{R}\left(F^{(k)}, p(t)\right) e^{\alpha w_{k}}\right)=\lim _{k \rightarrow \infty} \mathscr{E}\left(u^{(k)}, h_{R}\left(F^{(k)}, p(t)\right) e^{\alpha w_{k}}\right) .
$$

Proof Since $u^{(k)}$ converges to $u_{t}$ in $\mathbb{D}$ as $k \rightarrow \infty$, proving that the sequence $\left\{h_{R}\left(F^{(k)}, p(t)\right) e^{\alpha w_{k}}\right\}_{k=1}^{\infty}$ is bounded in $\mathbb{D}$ suffices. Boundedness in $L^{2}(\mu)$ is straightforward. For each $k$,

$$
\begin{aligned}
& \mathscr{E} 0\left(h_{R}\left(F^{(k)}, p(t)\right) e^{\alpha w_{k}}\right)^{1 / 2} \\
\leq & \mathscr{E}^{0}\left(h_{R}\left(F^{(k)}, p(t)\right)\left(e^{\alpha w_{k}}-1\right)\right)^{1 / 2}+\mathscr{E}^{0}\left(h_{R}\left(F^{(k)}, p(t)\right)\right)^{1 / 2} \\
\leq \| & \left\|g_{R}\left(F^{(k)}, p(t)\right)^{2}\left(e^{\alpha w_{k}}-1\right)\left|D F^{(k)}\right|_{H}\right\|_{2}+\left\|h_{R}\left(F^{(k)}, p(t)\right) \alpha e^{\alpha w_{k}}\left|D w_{k}\right|_{H}\right\|_{2} \\
& \quad+\left\|g_{R}\left(F^{(k)}, p(t)\right)^{2}\left|D F^{(k)}\right|_{H}\right\|_{2} .
\end{aligned}
$$

We note that $g_{R}$ is a bounded function, that $\left|e^{\alpha w_{k}}-1\right| \leq e^{|\alpha| N}$, that $h_{R}\left(F^{(k)}, p(t)\right)=0$ on $E \backslash E_{k}$, that $\left|D w_{k}\right|_{H} \leq 1$ on $E_{k}$ and that

$$
\begin{aligned}
\left\|\left|D F^{(k)}\right|_{H}\right\|_{2} & \leq\left\|\alpha e^{\alpha w_{k}} u^{(k)}\left|D w_{k}\right|_{H}\right\|_{2}+\left\|e^{\alpha w_{k}}\left|D u^{(k)}\right|_{H}\right\|_{2} \\
& \leq|\alpha| e^{|\alpha| N}\left\|u^{(k)}\right\|_{2}+e^{|\alpha| N}\left(2 \mathscr{E}^{0}\left(u^{(k)}\right)\right)^{1 / 2},
\end{aligned}
$$


which is bounded in $k$. From these estimates, the first and third terms of Eq. 3.19 are bounded in $k$. Moreover, Lemma 3.3(ii) and the inequality $2<2(q-1)<q_{0}$ together imply that $\left\{h_{R}\left(F^{(k)}, p(t)\right)\right\}_{k=1}^{\infty}$ is bounded in $L^{2}(\mu)$. Thus, the second term of Eq. 3.19 is also bounded in $k$, which completes the proof.

From this lemma and Eq. 3.18,

$$
\int_{E} \zeta(F(t), p(t)) e^{\alpha w} \mathscr{L} u_{t} d \mu=\lim _{R \rightarrow \infty} \lim _{k \rightarrow \infty}-\mathscr{E}\left(u^{(k)}, h_{R}\left(F^{(k)}, p(t)\right) e^{\alpha w_{k}}\right) .
$$

We provide an upper estimate of the right-hand side. Let $G_{R}^{(k)}=\rho_{R}\left(F^{(k)}, p(t)\right)$ for $k \in \mathbb{N}$. For the moment, we omit $p(t)$ from the notation and write, for example, $h_{R}\left(F^{(k)}\right)$ instead of $h_{R}\left(F^{(k)}, p(t)\right)$. We have

$$
\begin{aligned}
& -\mathscr{E}\left(u^{(k)}, h_{R}\left(F^{(k)}\right) e^{\alpha w_{k}}\right) \\
& =-\mathscr{E}\left(e^{-\alpha w_{k}} F^{(k)}, e^{\alpha w_{k}} h_{R}\left(F^{(k)}\right)\right) \\
& =-\mathscr{E}^{0}\left(e^{-\alpha w_{k}} F^{(k)}, e^{\alpha w_{k}} h_{R}\left(F^{(k)}\right)\right)-\int_{E}\left(b, D\left(e^{-\alpha w_{k}} F^{(k)}\right)\right)_{H} e^{\alpha w_{k}} h_{R}\left(F^{(k)}\right) d \mu \\
& -\int_{E}\left(c, D\left(e^{\alpha w_{k}} h_{R}\left(F^{(k)}\right)\right)\right)_{H} e^{-\alpha w_{k}} F^{(k)} d \mu-\int_{E} V F^{(k)} h_{R}\left(F^{(k)}\right) d \mu \\
& =-\frac{1}{2} \int_{E}\left(-\alpha e^{-\alpha w_{k}} F^{(k)} D w_{k}+e^{-a w_{k}} D F^{(k)}\right. \text {, } \\
& \left.\alpha e^{\alpha w_{k}} h_{R}\left(F^{(k)}\right) D w_{k}+e^{\alpha w_{k}} g_{R}\left(F^{(k)}\right)^{2} D F^{(k)}\right)_{H} d \mu \\
& -\int_{E}\left(b,-\alpha e^{-\alpha w_{k}} F^{(k)} D w_{k}+e^{-\alpha w_{k}} D F^{(k)}\right)_{H} e^{\alpha w_{k}} h_{R}\left(F^{(k)}\right) d \mu \\
& -\int_{E}\left(c, \alpha e^{\alpha w_{k}} h_{R}\left(F^{(k)}\right) D w_{k}+e^{\alpha w_{k}} g_{R}\left(F^{(k)}\right)^{2} D F^{(k)}\right)_{H} e^{-\alpha w_{k}} F^{(k)} d \mu \\
& -\int_{E} V F^{(k)} h_{R}\left(F^{(k)}\right) d \mu \\
& =-\frac{1}{2} \int_{E}\left\{g_{R}\left(F^{(k)}\right)^{2}\left|D F^{(k)}\right|_{H}^{2}-\alpha^{2} F^{(k)} h_{R}\left(F^{(k)}\right)\left|D w_{k}\right|_{H}^{2}\right. \\
& \left.+\alpha\left(h_{R}\left(F^{(k)}\right)-F^{(k)} g_{R}\left(F^{(k)}\right)^{2}\right)\left(D F^{(k)}, D w_{k}\right)_{H}\right\} d \mu \\
& +\alpha \int_{E}\left(b-c, D w_{k}\right)_{H} F^{(k)} h_{R}\left(F^{(k)}\right) d \mu \\
& +\frac{1}{2} \int_{E}\left(b-c, D F^{(k)}\right)_{H}\left(F^{(k)} g_{R}\left(F^{(k)}\right)^{2}-h_{R}\left(F^{(k)}\right)\right) d \mu \\
& -\frac{1}{2} \int_{E}\left(b+c, D F^{(k)}\right)_{H}\left(F^{(k)} g_{R}\left(F^{(k)}\right)^{2}+h_{R}\left(F^{(k)}\right)\right) d \mu \\
& -\int_{E} V F^{(k)} h_{R}\left(F^{(k)}\right) d \mu \\
& =: I_{1}+I_{2}+I_{3}+I_{4}+I_{5} \text {. }
\end{aligned}
$$


Using Eq. 3.14, we have

$$
\begin{aligned}
& I_{1} \leq-\frac{1}{2} \int_{E} g_{R}\left(F^{(k)}\right)^{2}\left|D F^{(k)}\right|_{H}^{2} d \mu \\
& +\frac{\alpha^{2}(1+\varepsilon)}{2} \int_{E} \rho_{R}\left(F^{(k)}\right)^{2}\left|D w_{k}\right|_{H}^{2} d \mu \quad \text { (from Lemma 3.8(iii)) } \\
& +\frac{|\alpha| \varepsilon}{2} \int_{E} \rho_{R}\left(F^{(k)}\right) g_{R}\left(F^{(k)}\right)\left|\left(D F^{(k)}, D w_{k}\right)_{H}\right| d \mu \\
& \text { (from Lemma 3.4(i) and Lemma 3.8(iv)) } \\
& \leq-\frac{1}{2} \int_{E}\left|D G_{R}^{(k)}\right|_{H}^{2} d \mu+\frac{\alpha^{2}(1+\varepsilon)}{2} \int_{E}\left(G_{R}^{(k)}\right)^{2} d \mu+\frac{|\alpha| \varepsilon}{2} \int_{E} G_{R}^{(k)}\left|D G_{R}^{(k)}\right|_{H} d \mu \\
& \leq-\mathscr{E}^{0}\left(G_{R}^{(k)}\right)+\frac{\alpha^{2}(1+\varepsilon)}{2}\left\|G_{R}^{(k)}\right\|_{2}^{2}+\varepsilon\left\|D G_{R}^{(k)}\right\|_{2}^{2}+\frac{\varepsilon \alpha^{2}}{16}\left\|G_{R}^{(k)}\right\|_{2}^{2}, \\
& I_{3}=\int_{E}\left(b-c, D \sqrt{\iota_{R}\left(F^{(k)}\right)}\right)_{H} \sqrt{\iota_{R}\left(F^{(k)}\right)} d \mu \\
& \leq \kappa \mathscr{E}_{1}^{0}\left(\sqrt{\iota_{R}\left(F^{(k)}\right)}\right) \quad(\text { from }(\mathrm{B} .1)) \\
& \leq \kappa K_{0}^{2}(p(t)-2)\left(\frac{1}{2} \int_{E}\left|D F^{(k)}\right|_{H}^{2} g_{R}\left(F^{(k)}\right)^{2} d \mu+\left\|G_{R}^{(k)}\right\|_{2}^{2}\right) \quad \text { (from Lemma 3.5) } \\
& \leq \varepsilon \mathscr{E}_{1}^{0}\left(G_{R}^{(k)}\right), \\
& I_{4}+I_{5}=-\int_{E}\left(b+c, D\left(\psi_{R}\left(F^{(k)}\right)\right)\right)_{H} \psi_{R}\left(F^{(k)}\right) d \mu-\int_{E} V \psi_{R}\left(F^{(k)}\right)^{2} d \mu \\
& \leq \eta \mathscr{E}^{0}\left(\psi_{R}\left(F^{(k)}\right)\right)+\theta\left\|\psi_{R}\left(F^{(k)}\right)\right\|_{2}^{2} \quad \text { (from (A.2)) } \\
& \leq \frac{\eta(1+2 \delta)}{2} \int_{E}\left|D F^{(k)}\right|_{H}^{2} g_{R}\left(F^{(k)}\right)^{2} d \mu+\theta(1+\delta)^{2}\left\|G_{R}^{(k)}\right\|_{2}^{2} \\
& \text { (from Lemma 3.8(vi) and (iii)) } \\
& \leq(1+\varepsilon) \eta \mathscr{E}^{0}\left(G_{R}^{(k)}\right)+(1+\varepsilon) \theta\left\|G_{R}^{(k)}\right\|_{2}^{2} .
\end{aligned}
$$

Moreover, when $\alpha>0$,

$$
\begin{aligned}
& I_{2}= \alpha \int_{E}(b-c, D w)_{H} \psi_{R}\left(F^{(k)}\right)^{2} d \mu \\
&= \alpha \int_{\left\{0<\mathrm{d}_{B}<\mathrm{d}(A, B)\right\}}\left(b-c, D \mathrm{~d}_{B}\right)_{H} \psi_{R}\left(F^{(k)}\right)^{2} d \mu \quad \text { (from Proposition 2.1) } \\
& \leq \alpha\left\{\frac{\varepsilon}{\alpha} \mathscr{E}^{0}\left(\psi_{R}\left(F^{(k)}\right)\right)+\lambda_{\varepsilon / \alpha}\left\|\psi_{R}\left(F^{(k)}\right)\right\|_{2}^{2}\right. \\
&\left.\quad+\gamma\left\|\psi_{R}\left(F^{(k)}\right)\right\|_{2}\left(\int_{E} \psi_{R}\left(F^{(k)}\right)^{2} \log ^{+} \frac{\psi_{R}\left(F^{(k)}\right)^{2}}{\left\|\psi_{R}\left(F^{(k)}\right)\right\|_{2}^{2}} d \mu\right)^{1 / 2}\right\} \\
& \leq \varepsilon(1+\varepsilon) \mathscr{E}^{0}\left(G_{R}^{(k)}\right)+\alpha \lambda_{\varepsilon / \alpha}(1+\varepsilon)\left\|G_{R}^{(k)}\right\|_{2}^{2} \quad\left(\text { from }(\mathrm{B} .2)_{A, B} \text { with } f=\psi_{R}\left(F^{(k)}\right) /\left\|\psi_{R}\left(F^{(k)}\right)\right\|_{2}\right) \\
&+\varepsilon \alpha^{2}(1+\varepsilon)\left\|G_{R}^{(k)}\right\|_{2}^{2}+\frac{\gamma^{2}}{4 \varepsilon} \int_{E} \psi_{R}\left(F^{(k)}\right)^{2} \log ^{+} \frac{\psi_{R}\left(F^{(k)}\right)^{2}}{\left\|\psi_{R}\left(F^{(k)}\right)\right\|_{2}^{2}} d \mu . \\
& \quad(\text { from Lemma 3.8(vi) and (iii)) }
\end{aligned}
$$


Assume $\alpha>0$ in what follows. Combining Eqs. 3.16, 3.20, and the estimates from $I_{1}$ to $I_{5}$ above, we have

$$
\begin{aligned}
\sigma^{\prime}(t)=\lim _{R \rightarrow \infty} \lim _{k \rightarrow \infty}\left(-\mathscr{E}\left(u^{(k)}, h_{R}\left(F^{(k)}, p(t)\right) e^{\alpha w_{k}}\right)-S \int_{E} \partial_{y} \tau(F(t), p(t)) d \mu\right) \\
\leq \lim _{R \rightarrow \infty} \underset{k \rightarrow \infty}{\lim }\left[C_{0} \mathscr{E}^{0}\left(G_{R}^{(k)}\right)+C_{1}\left\|G_{R}^{(k)}\right\|_{2}^{2}+\frac{\gamma^{2}}{4 \varepsilon} \int_{E} \psi_{R}\left(F^{(k)}\right)^{2} \log ^{+} \frac{\psi_{R}\left(F^{(k)}\right)^{2}}{\left\|\psi_{R}\left(F^{(k)}\right)\right\|_{2}^{2}} d \mu\right. \\
\left.-S \int_{E}\left\{F(t)^{p(t)} \log ^{+} F(t)-2(\log 2) F(t)^{2}\right\} d \mu\right], \quad \text { (from Lemma 3.6) }
\end{aligned}
$$

where

$$
C_{0}=-1+3 \varepsilon+(1+\varepsilon) \eta+\varepsilon(1+\varepsilon) \leq 0 \quad \text { (by the choice of } \varepsilon \text { ) }
$$

and

$$
C_{1}=\frac{(1+\varepsilon) \alpha^{2}}{2}+\frac{\varepsilon \alpha^{2}}{16}+\varepsilon+(1+\varepsilon) \theta+(1+\varepsilon) \alpha \lambda_{\varepsilon / \alpha}+\varepsilon(1+\varepsilon) \alpha^{2} .
$$

Let $G(t)=\sqrt{\tau(F(t), p(t))}$. Note that $\|G(t)\|_{2}^{2}=\sigma(t)$. From Lemmas 3.8(iii)(v) and 3.3(ii),

$$
\begin{aligned}
& 0 \leq \psi_{R}\left(F^{(k)}\right) \leq \sqrt{1+\varepsilon} G_{R}^{(k)} \leq \sqrt{2}(1+\varepsilon) G(t) \leq 2 G(t) \quad \mu \text {-a.e. } \\
& \text { for all } k \in \mathbb{N} \text { and } R>2
\end{aligned}
$$

and

$$
F(t) \leq G(t) \leq F(t) \vee F(t)^{p(t) / 2} .
$$

Since $\log ^{+}(x / y) \leq \log ^{+} x+\log ^{-} y$ for $x, y>0$ and the maps $[0, \infty) \ni a \mapsto a \log ^{+} a \in$ $[0, \infty)$ and $[0, \infty) \ni a \mapsto a+a \log ^{-} a \in[0, \infty)$ are both non-decreasing, we obtain, for every $k \in \mathbb{N}$ and $R>2$, that

$$
\begin{aligned}
& \int_{E} \psi_{R}\left(F^{(k)}\right)^{2} \log ^{+} \frac{\psi_{R}\left(F^{(k)}\right)^{2}}{\left\|\psi_{R}\left(F^{(k)}\right)\right\|_{2}^{2}} d \mu \\
\leq & \int_{E} \psi_{R}\left(F^{(k)}\right)^{2} \log ^{+} \frac{\psi_{R}\left(F^{(k)}\right)^{2}}{4} d \mu+\left\|\psi_{R}\left(F^{(k)}\right)\right\|_{2}^{2} \log ^{-} \frac{\left\|\psi_{R}\left(F^{(k)}\right)\right\|_{2}^{2}}{4} \\
\leq & 4 \int_{E} G(t)^{2} \log ^{+} G(t)^{2} d \mu+4\left(\|G(t)\|_{2}^{2}+\|G(t)\|_{2}^{2} \log ^{-}\|G(t)\|_{2}^{2}\right)
\end{aligned}
$$

and

$$
\begin{aligned}
\int_{E} G(t)^{2} \log ^{+} G(t)^{2} d \mu & \leq \int_{E}\left(F(t)^{2} \vee F(t)^{p(t)}\right) \log ^{+}\left(F(t)^{2} \vee F(t)^{p(t)}\right) d \mu \\
& =p(t) \int_{E} F(t)^{p(t)} \log ^{+} F(t) d \mu
\end{aligned}
$$

Here, in the last equality, we used the identity

$$
\left(a^{2} \vee a^{p(t)}\right) \log ^{+}\left(a^{2} \vee a^{p(t)}\right)=p(t) a^{p(t)} \log ^{+} a \quad \text { for } a \geq 0 .
$$


Because we set $S=3 \gamma^{2} / \varepsilon$,

$$
\begin{aligned}
\sigma^{\prime}(t) \leq(2+ & 2 \varepsilon) C_{1}\|G(t)\|_{2}^{2}+\frac{p(t) S}{3} \int_{E} F(t)^{p(t)} \log ^{+} F(t) d \mu \\
& +\frac{S}{3}\left(\|G(t)\|_{2}^{2}+\|G(t)\|_{2}^{2} \log ^{-}\|G(t)\|_{2}^{2}\right) \\
& -S \int_{E} F(t)^{p(t)} \log ^{+} F(t) d \mu+2 S(\log 2)\|F(t)\|_{2}^{2} \\
\leq & U \sigma(t)+W \sigma(t) \log ^{-} \sigma(t),
\end{aligned}
$$

where

$$
U=(2+2 \varepsilon) C_{1}+\left(\frac{1}{3}+2 \log 2\right) S \quad \text { and } \quad W=\frac{S}{3} .
$$

\subsection{Solving the Differential Inequality}

We give an explicit upper bound of $\sigma(t)$. Since $\left\|u_{0}\right\|_{2}>0$, there exists some $t_{1} \in\left(0, t_{0}\right]$ such that $\left\|u_{t}\right\|_{2}>0$ for $t \in\left[0, t_{1}\right]$. For this step, we consider only $t \in\left[0, t_{1}\right]$. Keeping in mind that

$$
(\log \sigma)^{\prime}(t)=\sigma^{\prime}(t) / \sigma(t) \leq U+W \log ^{-} \sigma(t)
$$

from Eq. 3.25, we define

$$
\chi(x)=\int_{0}^{x} \frac{1}{U+W \max \{-s, 0\}} d s= \begin{cases}-\frac{1}{W} \log \left(1-\frac{W}{U} x\right) & (x \leq 0), \\ \frac{x}{U} & (x>0) .\end{cases}
$$

Then,

$$
(\chi(\log \sigma))^{\prime}(t)=\chi^{\prime}(\log \sigma(t))(\log \sigma)^{\prime}(t) \leq 1,
$$

which implies

$$
\chi(\log \sigma(t)) \leq \chi(\log \sigma(0))+t, \quad t \geq 0 .
$$

This inequality implies

$$
\log \sigma(t) \leq \begin{cases}\frac{U}{W}[1-\exp (-W\{\chi(\log \sigma(0))+t\})] & \text { if } \sigma(t) \leq 1 \\ U\{\chi(\log \sigma(0))+t\} & \text { if } \sigma(t)>1\end{cases}
$$

We can confirm that

$$
\frac{U}{W}[1-\exp (-W z)] \leq U z, \quad z \in \mathbb{R},
$$

so that Eq. 3.28 implies

$$
\sigma(t) \leq \exp (U\{\chi(\log \sigma(0))+t\}) .
$$

For the proof of Theorem 2.10, we assume that $\mathrm{d}(A, B)>0$ because otherwise the assertion is trivial. Define

$$
\sigma_{1}(t, \alpha)=\int_{E} \tau\left(e^{\alpha w} T_{t} \mathbf{1}_{B}, p(t)\right) d \mu \quad \text { and } \quad \sigma_{2}(t, \alpha)=\int_{E} \tau\left(e^{-\alpha w} \hat{T}_{t} \mathbf{1}_{A}, p(t)\right) d \mu
$$

for $t>0$ and $\alpha>0$. Both $\sigma_{1}(t, \alpha)$ and $\sigma_{2}(t, \alpha)$ have the same kind of estimates as Eq. 3.29. Indeed, for the estimate of $\sigma_{2}$, the discussion in the previous subsection is applied with $b$, $c$, and $\alpha$ replaced by $c, b$, and $-\alpha$, respectively. The only term that requires care is $I_{2}$, but 
the estimate (3.21) is unchanged by this replacement. From the Cauchy-Schwarz inequality and Lemma 3.3(ii),

$$
\begin{aligned}
P_{t}(A, B) & \leq\left\{\int_{E}\left(e^{\alpha w} T_{t / 2} \mathbf{1}_{B}\right)^{2} d \mu\right\}^{1 / 2}\left\{\int_{E}\left(e^{-\alpha w} \hat{T}_{t / 2} \mathbf{1}_{A}\right)^{2} d \mu\right\}^{1 / 2} \\
& \leq \sigma_{1}(t / 2, \alpha)^{1 / 2} \sigma_{2}(t / 2, \alpha)^{1 / 2}
\end{aligned}
$$

Letting $N=\mathrm{d}(A, B)$ and $\alpha=N / t$, we have

$$
\varlimsup_{t \rightarrow 0} t \log P_{t}(A, B) \leq \varlimsup_{t \rightarrow 0} \frac{t}{2} \log \sigma_{1}(t / 2, N / t)+\varlimsup_{t \rightarrow 0} \frac{t}{2} \log \sigma_{2}(t / 2, N / t) .
$$

We also have

$$
\begin{aligned}
\sigma_{1}(0, N / t) & =\int_{E} \tau\left(\mathbf{1}_{B}, q\right) d \mu=\mu(B), \\
\sigma_{2}(0, N / t) & =\int_{E} \tau\left(e^{-N^{2} / t} \mathbf{1}_{A}, q\right) d \mu=\mu(A) e^{-2 N^{2} / t}, \\
\frac{t}{2} \log \sigma_{j}\left(\frac{t}{2}, \frac{N}{t}\right) & =\frac{U t}{2} \chi\left(\log \sigma_{j}\left(0, \frac{N}{t}\right)\right)+\frac{U t^{2}}{4}, \quad j=1,2 .
\end{aligned}
$$

We remark that $U$ and $\chi$ depend on $\alpha$ (see Eqs. 3.26 and 3.22). When $\alpha=N / t$,

$$
\begin{aligned}
\lim _{t \rightarrow 0} U t^{2} & =(2+2 \varepsilon)\left(\frac{1+\varepsilon}{2}+\frac{\varepsilon}{16}+\varepsilon\right) N^{2} \quad \text { (from Eq. 2.6) } \\
& =: \beta(\varepsilon) N^{2}
\end{aligned}
$$

and

$$
\lim _{t \rightarrow 0} U \chi(x)=x \quad \text { for } x \in \mathbb{R}
$$

in view of Eq. 3.27. In particular, $U=O\left(t^{-2}\right)$ as $t \rightarrow 0$. We also remark that $\lim _{\varepsilon \rightarrow 0} \beta(\varepsilon)=1$. Then, we obtain

$$
\frac{U t}{2} \chi\left(\log \sigma_{1}\left(0, \frac{N}{t}\right)\right)+\frac{U t^{2}}{4}=\frac{t}{2} U \chi(\log \mu(B))+\frac{U t^{2}}{4} \rightarrow \frac{\beta(\varepsilon) N^{2}}{4} \text { as } t \rightarrow 0 .
$$

Therefore,

$$
\varlimsup_{t \rightarrow 0} \frac{t}{2} \log \sigma_{1}(t / 2, N / t) \leq \frac{\beta(\varepsilon) N^{2}}{4} .
$$

Also, for $t$ small enough that $\sigma_{2}(0, N / t)<1$,

$$
\begin{aligned}
\frac{U t}{2} \chi\left(\log \sigma_{2}\left(0, \frac{N}{t}\right)\right)+\frac{U^{2} t}{4} & =\frac{t}{2}\left(-\frac{U}{W} \log \left(1-\frac{W}{U}\left(\log \mu(A)-\frac{2 N^{2}}{t}\right)\right)+\frac{U t}{2}\right) \\
& =\frac{U t}{2 W}\left(-\frac{2 W N^{2}}{U t}+O\left(t^{2}\right)\right)+\frac{U t^{2}}{4} \\
& \rightarrow-N^{2}+\frac{\beta(\varepsilon)}{4} N^{2} \quad \text { as } t \rightarrow 0 .
\end{aligned}
$$

Therefore,

$$
\varlimsup_{t \rightarrow 0} \frac{t}{2} \log \sigma_{2}(t / 2, N / t) \leq\left(-1+\frac{\beta(\varepsilon)}{4}\right) N^{2} .
$$

By combining Eqs. 3.32, 3.33, and 3.34,

$$
\varlimsup_{t \rightarrow 0} t \log P_{t}(A, B) \leq\left(-1+\frac{\beta(\varepsilon)}{2}\right) N^{2} .
$$


Letting $\varepsilon \rightarrow 0$, we obtain Eq. 2.8, which finishes the proof of Theorem 2.10.

Remark 3.11 (i) As seen from the proof, when we can let $\gamma=0$ in (B.2) ${ }_{A, B}$, the $L^{p}$ analysis is not necessary and the proof becomes much simpler.

(ii) If $(E, \mathscr{B}, \mu)$ is a finite measure space, then we can define $\sigma, \sigma_{1}$, and $\sigma_{2}$ as

$$
\begin{aligned}
\sigma(t) & =\int_{E} F(t)^{p(t)} d \mu, \\
\sigma_{1}(t, \alpha) & =\int_{E}\left(e^{\alpha w} T_{t} \mathbf{1}_{B}\right)^{p(t)} d \mu, \\
\sigma_{2}(t, \alpha) & =\int_{E}\left(e^{-\alpha w} \hat{T}_{t} \mathbf{1}_{A}\right)^{p(t)} d \mu
\end{aligned}
$$

and use the inequality

$$
P_{t}(A, B) \leq \mu(E)^{1-\frac{2}{p(t / 2)}} \sigma_{1}(t / 2, \alpha)^{\frac{1}{p(t / 2)}} \sigma_{2}(t / 2, \alpha)^{\frac{1}{p(t / 2)}}
$$

in place of Eqs. 3.15, 3.30, and 3.31. This change makes the proof of Theorem 2.10 shorter and simpler since the fine estimates in Section 3.2 are not necessary.

\section{Proof of Theorem 2.11}

\subsection{Cutoff Functions and Their Properties}

We turn to the lower-side estimate and prove Theorem 2.11. In Section 2.1 of [9], some nice concave functions are introduced as cutoff functions. Because our semigroup $\left\{T_{t}\right\}_{t>0}$ does not have the Markov property in general, we need to modify these functions to be suitable. First, we take a real-valued function $g$ on $\mathbb{R}$ satisfying the following properties:

- $\quad g$ is an odd and bounded $C^{3}$-function;

$-g(x)=x$ for $x \in[-1,1]$ and $0<g^{\prime}(x) \leq 1$ on $\mathbb{R}$; and

- there is a positive constant $C$ such that $0 \leq-g^{\prime \prime}(x) \leq C g^{\prime}(x)$ for $x \in[-1, \infty)$.

These conditions imply that $\lim _{x \rightarrow \infty} g(x)=L, \lim _{x \rightarrow-\infty} g(x)=-L$ for some $L>1$ and that the convergence is monotone. Note that $g$ is concave on $[-1, \infty)$.

Define our main cutoff functions at level $K>0$ by

$$
\phi^{K}(x)=K g(x / K), \Phi^{K}(x)=\int_{0}^{x}\left(\phi^{K}\right)^{\prime}(s)^{2} d s, \text { and } \Psi^{K}(x)=x\left(\phi^{K}\right)^{\prime}(x)^{2} .
$$

From the conditions on $g$, we have the following properties:

(C.1) $0<\left(\phi^{K}\right)^{\prime}(x) \leq 1$,

(C.2) $0 \leq\left|\left(\phi^{K}\right)^{\prime \prime}(x)\right| \leq C K^{-1}\left(\phi^{K}\right)^{\prime}(x)$,

(C.3) $0 \leq \Psi^{K}(x) \leq \Phi^{K}(x) \leq \phi^{K}(x) \leq L K$ for $x \geq 0$, and $\left|\Psi^{K}(x)\right|,\left|\Phi^{K}(x)\right|$, $\left|\phi^{K}(x)\right| \leq L K$ on $\mathbb{R}$,

(C.4) $\phi^{K}(x)=\Phi^{K}(x)=\Psi^{K}(x)=x$ on $[-K, K]$,

(C.5) $\lim _{x \rightarrow \infty} \Psi^{K}(x)=0$,

(C.6) $\Phi^{K}(x / \beta) \geq \Phi^{K}(x) / \beta$ for all $\beta>1$ and $x \geq 0$. 
To simplify the notation, we omit explicit indication of the dependency on $K$ for most of this section. For example, we write $\phi$ instead of $\phi^{K}$ whenever the value of $K$ is clear from the context. The monotonicities of $\phi, \Phi,(\mathrm{C} .3)$, and (C.5) guarantee that $\phi, \Phi$, and $\Psi$ can be extended to continuous functions on $[-\infty, \infty]$, and these extensions use the same symbols. The following estimates result:

$$
\Phi^{K}(x)-\Phi^{K}\left(\Phi^{M}(x)\right) \leq \Phi^{K}(\infty)-\Phi^{K}(M) \text { for all } K, M>0 \text { and } x \in \mathbb{R} .
$$

Indeed, this inequality is trivial when $x \leq M$, and when $x>M$ it is deduced from $\Phi^{K}(x) \leq$ $\Phi^{K}(\infty)$ and $\Phi^{K}\left(\Phi^{M}(x)\right) \geq \Phi^{K}(M)$.

We also introduce a function $\hat{\Phi}^{K}$ on $\mathbb{R}$, defining it as

$$
\hat{\Phi}^{K}(x)= \begin{cases}\Phi^{K}(x) & (x \geq-K), \\ x & (x<-K) .\end{cases}
$$

This function is concave and 1-Lipschitz on $\mathbb{R}$.

For functions $u_{t}^{\delta}$ on $E$ with parameters $t$ and $\delta$, we write $\phi_{t}^{\delta}$ for $\phi\left(u_{t}^{\delta}\right), \Phi_{t}^{\delta}$ for $\Phi\left(u_{t}^{\delta}\right), \bar{\phi}_{t}^{\delta}$ for $t^{-1} \int_{0}^{t} \phi_{s}^{\delta} d s$, and so on. We denote $\int_{E} f g d \mu$ by $(f, g)_{\mu}$ for functions $f$ and $g$ on $E$.

For $\delta \in(0,1]$ and $f \in L^{2}(\mu)$ with $f \geq 0 \mu$-a.e. and $t>0$, we define

$$
u_{t}^{\delta}(x)=-t \log \left(T_{t} f(x)+\delta\right), \quad e_{t}^{\delta}=-t \log \delta .
$$

We need the following lemmas, introduced in [9].

Lemma 4.1 The function $F(x)=\Phi(-t \log x)$ is convex for $x \in[0, \infty]$ if $0<t \leq$ $K /(2 C)$.

Proof The proof here follows the proof of Lemma 2.1 in [9]. Compute

$$
\begin{aligned}
F^{\prime}(x) & =-\frac{t}{x} \Phi^{\prime}(-t \log x), \\
F^{\prime \prime}(x) & =\frac{t}{x^{2}} \Phi^{\prime}(-t \log x)+\frac{t^{2}}{x^{2}} \Phi^{\prime \prime}(-t \log x)=\left.\frac{t}{x^{2}}\left(\left(\phi^{\prime}\right)^{2}+2 t \phi^{\prime} \phi^{\prime \prime}\right)\right|_{-t \log x} .
\end{aligned}
$$

From this and (C.2), $F$ has a nonnegative second derivative.

Lemma 4.2 ([9, Lemma 2.2]) Suppose that $F$ is a concave continuous function defined on $\mathbb{R}$. If $f_{n} \rightarrow f$ weakly in $L^{2}(\mu), F\left(f_{n}\right) \in L^{2}(\mu)$ for each $n$, and $F\left(f_{n}\right)$ has a subsequence that converges to some function $\hat{F}$ weakly in $L^{2}(\mu)$, then $\hat{F} \leq F(f)$.

Lemma 4.3 ([9, Lemma 2.4]) Let $\left\{f_{n}\right\}$ be a sequence of $\mathbb{D}$ that converges weakly to some $f$ in $\mathbb{D}$. Then,

$$
\varliminf_{n \rightarrow \infty} \int_{E}\left|D f_{n}\right|_{H}^{2} h d \mu \leq \int_{E}|D f|_{H}^{2} h d \mu
$$

for $h \in \mathbb{D}$ with $0 \leq h \leq M \mu$-a.e. for some $M \geq 0$.

Lemma 4.4 (cf. [9, Lemma 2.5]) Let $T>0$ and suppose that $f(t, x)=f_{t}(x)$ is a bounded jointly measurable function for $(t, x) \in(0, T] \times E$. Also suppose that $f_{t} \in \mathbb{D}$ for each 
$t \in(0, T]$ and that $\int_{0}^{T} \mathscr{E} 0\left(f_{t}\right) d t<\infty$. Writing $\bar{f}_{T}=\frac{1}{T} \int_{0}^{T} f_{t} d t$, we have $\bar{f}_{T} \in \mathbb{D}$, and the following is true for any nonnegative $h \in \mathbb{D}_{b}$ :

$$
\int_{E}\left|D \bar{f}_{T}\right|_{H}^{2} h d \mu \leq \frac{1}{T} \int_{0}^{T} \int_{E}\left|D f_{t}\right|_{H}^{2} h d \mu d t .
$$

\subsection{Rough Estimates}

In the following, $\Gamma(f, g)$ and $\Gamma(f)$ denote $(D f, D g)_{H}$ and $(D f, D f)_{H}$, respectively.

Lemma $4.5 u_{t}^{\delta}-e_{t}^{\delta} \in \mathbb{D}$ and

$$
\begin{aligned}
\left(\rho, \partial_{t} \Phi_{t}^{\delta}\right)_{\mu}=\frac{1}{t} & \left(\rho, \Psi_{t}^{\delta}\right)_{\mu}-\mathscr{E}^{0}\left(\left(\left(\phi^{\prime}\right)_{t}^{\delta}\right)^{2} \rho, u_{t}^{\delta}-e_{t}^{\delta}\right)-\frac{1}{2 t} \int_{E} \Gamma\left(u_{t}^{\delta}-e_{t}^{\delta}\right)\left(\left(\phi^{\prime}\right)_{t}^{\delta}\right)^{2} \rho d \mu \\
& -\int_{E}\left(b, D\left(u_{t}^{\delta}-e_{t}^{\delta}\right)\right)_{H}\left(\left(\phi^{\prime}\right)_{t}^{\delta}\right)^{2} \rho d \mu+t \int_{E}(c, D \rho)_{H} \frac{\left(\left(\phi^{\prime}\right)_{t}^{\delta}\right)^{2} T_{t} f}{T_{t} f+\delta} d \mu \\
& +\int_{E}\left(c, D\left(u_{t}^{\delta}-e_{t}^{\delta}\right)\right)_{H}\left\{2 t\left(\phi^{\prime}\right)_{t}^{\delta}\left(\phi^{\prime \prime}\right)_{t}^{\delta}+\left(\left(\phi^{\prime}\right)_{t}^{\delta}\right)^{2}\right\} \frac{\rho T_{t} f}{T_{t} f+\delta} d \mu \\
& +t \int_{E} V \frac{\left(\left(\phi^{\prime}\right)_{t}^{\delta}\right)^{2} \rho T_{t} f}{T_{t} f+\delta} d \mu
\end{aligned}
$$

for $\rho \in \bigcup_{k=1}^{\infty} \mathbb{D}_{E_{k}, b}$.

Proof Let $\Xi(s)=\log (s+\delta)-\log \delta$ for $s \in[0, \infty)$. From $\Xi(0)=0$, the boundedness of the derivative of $\Xi$ on $[0, \infty)$, and $u_{t}^{\delta}-e_{t}^{\delta}=-t \Xi\left(T_{t} f\right)$, we conclude that $u_{t}^{\delta}-e_{t}^{\delta} \in \mathbb{D}$.

We prove the identity (4.2). First,

$$
\left(\rho, \partial_{t} u_{t}^{\delta}\right)_{\mu}=\frac{1}{t}\left(\rho, u_{t}^{\delta}\right)_{\mu}-\left(\rho, \frac{t \mathscr{L} T_{t} f}{T_{t} f+\delta}\right)_{\mu}=\frac{1}{t}\left(\rho, u_{t}^{\delta}\right)_{\mu}+t \mathscr{E}\left(T_{t} f, \frac{\rho}{T_{t} f+\delta}\right)
$$

and

$$
\begin{aligned}
\mathscr{E}\left(\rho, \Xi\left(T_{t} f\right)\right)=\frac{1}{2} & \int_{E} \Gamma\left(\rho, \Xi\left(T_{t} f\right)\right) d \mu+\int_{E}(b, D \rho)_{H} \Xi\left(T_{t} f\right) d \mu \\
& +\int_{E}\left(c, D\left(\Xi\left(T_{t} f\right)\right)\right)_{H} \rho d \mu+\int_{E} V \rho \Xi\left(T_{t} f\right) d \mu .
\end{aligned}
$$

The first term on the right-hand side is computed as

$$
\begin{aligned}
& \frac{1}{2} \int_{E} \Gamma\left(\rho, \Xi\left(T_{t} f\right)\right) d \mu=\frac{1}{2} \int_{E} \Gamma\left(\rho, T_{t} f\right) \frac{1}{T_{t} f+\delta} d \mu \\
& =\frac{1}{2} \int_{E}\left\{\Gamma\left(\frac{\rho}{T_{t} f+\delta}, T_{t} f\right)+\Gamma\left(T_{t} f\right) \frac{\rho}{\left(T_{t} f+\delta\right)^{2}}\right\} d \mu \\
& =\mathscr{E}\left(T_{t} f, \frac{\rho}{T_{t} f+\delta}\right)-\int_{E}\left(b, D T_{t} f\right)_{H} \frac{\rho}{T_{t} f+\delta} d \mu \\
& \quad-\int_{E}\left(c, D\left(\frac{\rho}{T_{t} f+\delta}\right)\right)_{H} T_{t} f d \mu-\int_{E} V \frac{\rho T_{t} f}{T_{t} f+\delta} d \mu+\frac{1}{2} \int_{E} \Gamma\left(\Xi\left(T_{t} f\right)\right) \rho d \mu .
\end{aligned}
$$


Combining the identities Eqs. 4.3, 4.4, and 4.5, it holds that

$$
\begin{aligned}
\left(\rho, \partial_{t} u_{t}^{\delta}\right)_{\mu}=\frac{1}{t} & \left(\rho, u_{t}^{\delta}\right)_{\mu}+t \mathscr{E}\left(\rho, \Xi\left(T_{t} f\right)\right)+t \int_{E}\left(b, D T_{t} f\right)_{H} \frac{\rho}{T_{t} f+\delta} d \mu \\
& +t \int_{E}\left(c, D\left(\frac{\rho}{T_{t} f+\delta}\right)\right)_{H} T_{t} f d \mu+t \int_{E} V \frac{\rho T_{t} f}{T_{t} f+\delta} d \mu \\
& \quad-\frac{t}{2} \int_{E} \Gamma\left(\Xi\left(T_{t} f\right)\right) \rho d \mu-t \int_{E}(b, D \rho)_{H} \Xi\left(T_{t} f\right) d \mu \\
& -t \int_{E}\left(c, D\left(\Xi\left(T_{t} f\right)\right)\right)_{H} \rho d \mu-t \int_{E} V \rho \Xi\left(T_{t} f\right) d \mu \\
=\frac{1}{t} & \left(\rho, u_{t}^{\delta}\right)_{\mu}-\mathscr{E}\left(\rho, u_{t}^{\delta}-e_{t}^{\delta}\right)-\frac{1}{2 t} \int_{E} \Gamma\left(u_{t}^{\delta}-e_{t}^{\delta}\right) \rho d \mu \\
& +t \int_{E}\left(b, D T_{t} f\right)_{H} \frac{\rho}{T_{t} f+\delta} d \mu \\
& +t \int_{E}\left(c, D\left(\frac{\rho}{T_{t} f+\delta}\right)\right)_{H} T_{t} f d \mu+t \int_{E} V \frac{\rho T_{t} f}{T_{t} f+\delta} d \mu .
\end{aligned}
$$

By using the identity $\left(\rho, \partial_{t} \Phi_{t}^{\delta}\right)_{\mu}=\left(\left(\left(\phi^{\prime}\right)_{t}^{\delta}\right)^{2} \rho, \partial_{t} u_{t}^{\delta}\right)_{\mu}$ and replacing $\rho$ with $\left(\left(\phi^{\prime}\right)_{t}^{\delta}\right)^{2} \rho$ in the relation above, we obtain

$$
\begin{aligned}
\partial_{t}\left(\rho, \Phi_{t}^{\delta}\right)_{\mu}=\frac{1}{t} & \left(\left(\left(\phi^{\prime}\right)_{t}^{\delta}\right)^{2} \rho, u_{t}^{\delta}\right)_{\mu}-\mathscr{E}^{0}\left(\left(\left(\phi^{\prime}\right)_{t}^{\delta}\right)^{2} \rho, u_{t}^{\delta}-e_{t}^{\delta}\right) \\
& -\frac{1}{2 t} \int_{E} \Gamma\left(u_{t}^{\delta}-e_{t}^{\delta}\right)\left(\left(\phi^{\prime}\right)_{t}^{\delta}\right)^{2} \rho d \mu+t \int_{E}\left(b, D T_{t} f\right)_{H} \frac{\left(\left(\phi^{\prime}\right)_{t}^{\delta}\right)^{2} \rho}{T_{t} f+\delta} d \mu \\
& +t \int_{E}\left(c, D\left(\frac{\left(\left(\phi^{\prime}\right)_{t}^{\delta}\right)^{2} \rho}{T_{t} f+\delta}\right)\right)_{H} T_{t} f d \mu+t \int_{E} V \frac{\left(\left(\phi^{\prime}\right)_{t}^{\delta}\right)^{2} \rho T_{t} f}{T_{t} f+\delta} d \mu .
\end{aligned}
$$

Here, we have

$$
t \int_{E}\left(b, D T_{t} f\right)_{H} \frac{\left(\left(\phi^{\prime}\right)_{t}^{\delta}\right)^{2} \rho}{T_{t} f+\delta} d \mu=-\int_{E}\left(b, D\left(u_{t}^{\delta}-e_{t}^{\delta}\right)\right)_{H}\left(\left(\phi^{\prime}\right)_{t}^{\delta}\right)^{2} \rho d \mu
$$

and

$$
\begin{aligned}
& t \int_{E}\left(c, D\left(\frac{\left(\left(\phi^{\prime}\right)_{t}^{\delta}\right)^{2} \rho}{T_{t} f+\delta}\right)\right)_{H} T_{t} f d \mu \\
& =t \int_{E}\left(c, D\left(\left(\left(\phi^{\prime}\right)_{t}^{\delta}\right)^{2} \rho\right)\right)_{H} \frac{T_{t} f}{T_{t} f+\delta} d \mu+\int_{E}\left(c, D\left(u_{t}^{\delta}-e_{t}^{\delta}\right)\right)_{H} \frac{\left(\left(\phi^{\prime}\right)_{t}^{\delta}\right)^{2} \rho T_{t} f}{T_{t} f+\delta} d \mu \\
& =t \int_{E}(c, D \rho)_{H} \frac{\left(\left(\phi^{\prime}\right)_{t}^{\delta}\right)^{2} T_{t} f}{T_{t} f+\delta} d \mu \\
& \quad+\int_{E}\left(c, D\left(u_{t}^{\delta}-e_{t}^{\delta}\right)\right)_{H}\left\{2 t\left(\phi^{\prime}\right)_{t}^{\delta}\left(\phi^{\prime \prime}\right)_{t}^{\delta}+\left(\left(\phi^{\prime}\right)_{t}^{\delta}\right)^{2}\right\} \frac{\rho T_{t} f}{T_{t} f+\delta} d \mu .
\end{aligned}
$$

Substituting Eqs. 4.7 and 4.8 for Eq. 4.6, we obtain Eq. 4.2. 
Lemma 4.6 For $k \in \mathbb{N}$ and $\rho \in \mathbb{D}_{E_{k}, b}$ with $0 \leq \rho \leq 1 \mu$-a.e.,

$$
\begin{aligned}
& \left|\left(\rho, \partial_{t} \Phi_{t}^{\delta}\right)_{\mu}-\frac{1}{t}\left(\rho, \Psi_{t}^{\delta}\right)_{\mu}+\mathscr{E}^{0}\left(\left(\left(\phi^{\prime}\right)_{t}^{\delta}\right)^{2} \rho, u_{t}^{\delta}-e_{t}^{\delta}\right)+\frac{1}{2 t} \int_{E} \Gamma\left(u_{t}^{\delta}-e_{t}^{\delta}\right)\left(\left(\phi^{\prime}\right)_{t}^{\delta}\right)^{2} \rho d \mu\right| \\
& \leq\left(\frac{C t}{K}+1\right) \int_{E} \Gamma\left(u_{t}^{\delta}-e_{t}^{\delta}\right)\left(\left(\phi^{\prime}\right)_{t}^{\delta}\right)^{2} \rho d \mu+t \mathscr{E}^{0}(\rho)+R(t, k),
\end{aligned}
$$

where we define

$$
R(t, k):=\frac{1}{2} \int_{E_{k}}|b|_{H}^{2} d \mu+\left\{\left(\frac{C}{K}+\frac{1}{2}\right) t+\frac{1}{2}\right\} \int_{E_{k}}|c|_{H}^{2} d \mu+t \int_{E_{k}}|V| d \mu .
$$

Proof Using Proposition 2.1, (C.1), and (C.2), we have

$$
\begin{aligned}
& \left|\int_{E}\left(b, D\left(u_{t}^{\delta}-e_{t}^{\delta}\right)\right)_{H}\left(\left(\phi^{\prime}\right)_{t}^{\delta}\right)^{2} \rho d \mu\right| \leq \frac{1}{2} \int_{E_{k}}|b|_{H}^{2} d \mu+\frac{1}{2} \int_{E} \Gamma\left(u_{t}^{\delta}-e_{t}^{\delta}\right)\left(\left(\phi^{\prime}\right)_{t}^{\delta}\right)^{2} \rho d \mu, \\
& \left|\int_{E}(c, D \rho)_{H} \frac{\left(\left(\phi^{\prime}\right)_{t}^{\delta}\right)^{2} T_{t} f}{T_{t} f+\delta} d \mu\right| \leq \frac{1}{2} \int_{E_{k}}|c|_{H}^{2} d \mu+\mathscr{E}^{0}(\rho), \\
& \left|\int_{E}\left(c, D\left(u_{t}^{\delta}-e_{t}^{\delta}\right)\right)_{H}\left\{2 t\left(\phi^{\prime}\right)_{t}^{\delta}\left(\phi^{\prime \prime}\right)_{t}^{\delta}+\left(\left(\phi^{\prime}\right)_{t}^{\delta}\right)^{2}\right\} \frac{\rho T_{t} f}{T_{t} f+\delta} d \mu\right| \\
& \quad \leq\left(\frac{C t}{K}+\frac{1}{2}\right) \int_{E_{k}}|c|_{H}^{2} d \mu+\left(\frac{C t}{K}+\frac{1}{2}\right) \int_{E} \Gamma\left(u_{t}^{\delta}-e_{t}^{\delta}\right)\left(\left(\phi^{\prime}\right)_{t}^{\delta}\right)^{2} \rho d \mu, \\
& \left|\int_{E} \frac{\left(\left(\phi^{\prime}\right)_{t}^{\delta}\right)^{2} \rho T_{t} f}{T_{t} f+\delta} V d \mu\right| \leq \int_{E_{k}}|V| d \mu .
\end{aligned}
$$

These estimates and Lemma 4.5 together imply the claim.

Proposition 4.7 There exists a measurable nest $\left\{\hat{E}_{k}\right\}_{k=1}^{\infty}$ and functions $\left\{\chi_{k}\right\}_{k=1}^{\infty}$ in $\mathbb{D}$ such that, for every $k, \hat{E}_{k} \subset E_{k}, 0 \leq \chi_{k} \leq 1$ on $E, \chi_{k}=1$ on $\hat{E}_{k}$, and $\chi_{k}=0$ on $E \backslash E_{k}$.

Proof For $k \in \mathbb{N}$, there exists an $h_{k} \in \mathbb{D}$ such that $h_{k} \geq 1$ on $E_{k}$. Take a function $g_{k}$ from $\bigcup_{n=1}^{\infty} \mathbb{D}_{E_{n}}$ such that $\mathscr{E}_{1}^{0}\left(h_{k}-g_{k}\right) \leq 1 / k$, and set $Y_{k}=\left\{x \in E_{k} \mid g_{k}(x) \geq 1 / 2\right\}$. Let $\rho_{k}$ denote $0 \vee\left(2\left(h_{k}-g_{k}\right) \wedge 1\right)$. Then, $\mathscr{E}_{1}^{0}\left(\rho_{k}\right) \leq 4 / k$ and $\rho_{k}=1$ on $E_{k} \backslash Y_{k}$. We prove that $\bigcup_{k=1}^{\infty} \mathbb{D}_{Y_{k}}$ is dense in $\mathbb{D}$. Take $f \in \mathbb{D}_{E_{n}, b}$ for some $n \in \mathbb{N}$. For $k \geq n$, let $f_{k}=f-f \rho_{k}$. Then, $f_{k}=0 \mu$-a.e. on $\left(E \backslash E_{n}\right) \cup\left(E_{k} \backslash Y_{k}\right) \supset E \backslash Y_{k}$. It follows that $f_{k} \in \mathbb{D}_{Y_{k}}$. Moreover, since

$\mathscr{E}^{0}\left(f-f_{k}\right)^{1 / 2}=\mathscr{E}^{0}\left(f \rho_{k}\right)^{1 / 2} \leq\|f\|_{\infty} \mathscr{E}^{0}\left(\rho_{k}\right)^{1 / 2}+\mathscr{E}^{0}(f)^{1 / 2} \leq\|f\|_{\infty}(4 / k)^{1 / 2}+\mathscr{E}^{0}(f)^{1 / 2}$,

which is bounded in $k$, and

$$
\left\|f-f_{k}\right\|_{2} \leq\|f\|_{\infty}\left\|\rho_{k}\right\|_{2} \leq\|f\|_{\infty}(4 / k)^{1 / 2} \rightarrow 0 \quad(k \rightarrow \infty),
$$

the Cesàro means of a certain subsequence of $\left\{f_{k}\right\}_{k=n}^{\infty}$ belonging to $\bigcup_{k=n}^{\infty} \mathbb{D}_{Y_{k}}$ converge to $f$ in $\mathbb{D}$. Thus $\bigcup_{k=1}^{\infty} \mathbb{D}_{Y_{k}}$ is dense in $\mathbb{D}$ because $\bigcup_{n=1}^{\infty} \mathbb{D}_{E_{n}, b}$ is dense in $\mathbb{D}$.

Let $Z_{n}=\bigcup_{k=1}^{n} Y_{k}$ and define $\eta_{n}=0 \vee\left(2\left(g_{1} \vee \cdots \vee g_{n}\right) \wedge 1\right)$. Then, $Z_{n} \subset E_{n}$ and $\bigcup_{n=1}^{\infty} \mathbb{D}_{Z_{n}}$ is dense in $\mathbb{D}$. Moreover, $0 \leq \eta_{n} \leq 1$ on $E, \eta_{n}=1$ on $Z_{n}$ and $\eta_{n} \in \bigcup_{k=1}^{\infty} \mathbb{D}_{E_{k}}$. 
Take a strictly increasing sequence $\{m(n)\}_{n=1}^{\infty}$ such that $\eta_{n} \in \mathbb{D}_{E_{m(n)}}$ for every $n \in \mathbb{N}$ and define

$$
\begin{aligned}
& \hat{E}_{k}=\emptyset, \chi_{k}=0 \quad \text { for } 1 \leq k<m(1), \\
& \hat{E}_{k}=Z_{n}, \chi_{k}=\eta_{n} \quad \text { for } m(n) \leq k<m(n+1), n=1,2, \ldots
\end{aligned}
$$

Then, $\left\{\hat{E}_{k}\right\}_{k=1}^{\infty}$ and $\left\{\chi_{k}\right\}_{k=1}^{\infty}$ satisfy the required conditions.

Define $u_{t}^{\delta}=-t \log \left(T_{t} \mathbf{1}_{B}+\delta\right)$ for $B \in \mathscr{B}_{0}$.

Lemma 4.8 There exists a positive constant $T_{0}$, depending only on $C$ and $K$, such that both $\left\{\mathscr{E}^{0}\left(\bar{\phi}_{t}^{\delta} \chi_{k}\right)\right\}_{0<t \leq T_{0}, 0<\delta \leq 1}$ and $\left\{\mathscr{E}^{0}\left(\bar{\Phi}_{t}^{\delta} \chi_{k}\right)\right\}_{0<t \leq T_{0}, 0<\delta \leq 1}$ are bounded for each $k$.

Proof Let $U_{t}^{\delta}=2 \mathscr{E}^{\mathscr{0}}\left(\phi_{t}^{\delta} \chi_{k}\right), W_{t}^{\delta}=\int_{E} \Gamma\left(u_{t}^{\delta}-e_{t}^{\delta}\right)\left(\left(\phi^{\prime}\right)_{t}^{\delta}\right)^{2} \chi_{k}^{2} d \mu$, and $a_{k}=2 \mathscr{E}^{0}\left(\chi_{k}\right)$. Applying the chain rule (2.1),

$$
\begin{aligned}
U_{t}^{\delta} & =\int_{E} \Gamma\left(\phi_{t}^{\delta} \chi_{k}, \phi_{t}^{\delta} \chi_{k}\right) d \mu \\
& =\int_{E}\left\{\Gamma\left(u_{t}^{\delta}-e_{t}^{\delta}\right)\left(\left(\phi^{\prime}\right)_{t}^{\delta}\right)^{2} \chi_{k}^{2}+2 \Gamma\left(u_{t}^{\delta}-e_{t}^{\delta}, \chi_{k}\right) \phi_{t}^{\delta}\left(\phi^{\prime}\right)_{t}^{\delta} \chi_{k}+\Gamma\left(\chi_{k}\right)\left(\phi_{t}^{\delta}\right)^{2}\right\} d \mu \\
& \leq 2 W_{t}^{\delta}+2 K^{2} L^{2} a_{k} \quad(\text { from }(\mathrm{C} .3)) .
\end{aligned}
$$

Letting $\rho=\chi_{k}^{2}$ in Lemma 4.6,

$$
\begin{gathered}
W_{t}^{\delta} \leq-2 t\left(\chi_{k}^{2}, \partial_{t} \Phi_{t}^{\delta}\right)_{\mu}+2\left(\chi_{k}^{2}, \Psi_{t}^{\delta}\right)_{\mu}-2 t \mathscr{E}^{\circ 0}\left(\left(\left(\phi^{\prime}\right)_{t}^{\delta}\right)^{2} \chi_{k}^{2}, u_{t}^{\delta}-e_{t}^{\delta}\right) \\
+2 t\left(\frac{C t}{K}+1\right) W_{t}^{\delta}+t^{2} a_{k}+2 t R(t, k) .
\end{gathered}
$$

From (C.3), $\left(\chi_{k}^{2}, \Psi_{t}^{\delta}\right)_{\mu} \leq K L$. Moreover,

$$
\begin{aligned}
& -2 t \mathscr{E}^{0}\left(\left(\left(\phi^{\prime}\right)_{t}^{\delta}\right)^{2} \chi_{k}^{2}, u_{t}^{\delta}-e_{t}^{\delta}\right) \\
& =-2 t \int_{E}\left(\phi^{\prime}\right)_{t}^{\delta} \Gamma\left(\chi_{k}, \chi_{k} \phi_{t}^{\delta}\right) d \mu+2 t \int_{E}\left(\phi^{\prime}\right)_{t}^{\delta} \phi_{t}^{\delta} \Gamma\left(\chi_{k}\right) d \mu \\
& \quad-2 t \int_{E}\left(\phi^{\prime \prime}\right)_{t}^{\delta}\left(\phi^{\prime}\right)_{t}^{\delta} \chi_{k}^{2} \Gamma\left(u_{t}^{\delta}-e_{t}^{\delta}\right) d \mu \\
& \leq \int_{E}\left(8 t^{2} \Gamma\left(\chi_{k}\right)+\frac{1}{8} \Gamma\left(\chi_{k} \phi_{t}^{\delta}\right)\right) d \mu+2 K L t \int_{E} \Gamma\left(\chi_{k}\right) d \mu \\
& \quad+\frac{2 C t}{K} \int_{E}\left(\left(\phi^{\prime}\right)_{t}^{\delta}\right)^{2} \chi_{k}^{2} \Gamma\left(u_{t}^{\delta}-e_{t}^{\delta}\right) d \mu \quad \text { (from (C.1), (C.2), and (C.3)) } \\
& =8 t^{2} a_{k}+\frac{1}{8} U_{t}^{\delta}+2 K L t a_{k}+\frac{2 C t}{K} W_{t}^{\delta} .
\end{aligned}
$$

Thus, we have

$$
\begin{aligned}
W_{t}^{\delta} \leq- & t\left(\chi_{k}^{2}, \partial_{t} \Phi_{t}^{\delta}\right)_{\mu}+\left(9 t^{2}+2 K L t\right) a_{k}+U_{t}^{\delta} / 8 \\
& +\left(2 C t / K+2 C t^{2} / K+2 t\right) W_{t}^{\delta}+2 K L+2 t R(t, k) .
\end{aligned}
$$

Choose $T_{0}>0$ such that $2 C T_{0} / K+2 C T_{0}^{2} / K+2 T_{0} \leq 1 / 2$. Then, for $t \in\left(0, T_{0}\right]$,

$$
W_{t}^{\delta} \leq-4 t\left(\chi_{k}^{2}, \partial_{t} \Phi_{t}^{\delta}\right)_{\mu}+U_{t}^{\delta} / 4+2 C_{2},
$$


where $C_{2}:=\left(9 T_{0}^{2}+2 K L T_{0}\right) a_{k}+2 K L+2 T_{0} R\left(T_{0}, k\right)$. By putting this inequality into Eq. 4.9,

$$
U_{t}^{\delta} \leq-8 t\left(\chi_{k}^{2}, \partial_{t} \Phi_{t}^{\delta}\right)_{\mu}+U_{t}^{\delta} / 2+4 C_{2}+2 K^{2} L^{2} a_{k},
$$

so that

$$
U_{t}^{\delta} \leq-16 t \partial_{t}\left(\chi_{k}^{2}, \Phi_{t}^{\delta}\right)_{\mu}+2 C_{3},
$$

where $C_{3}=4 C_{2}+2 K^{2} L^{2} a_{k}$. Therefore,

$$
\begin{aligned}
\int_{\varepsilon}^{t} \mathscr{E}^{0}\left(\phi_{s}^{\delta} \chi_{k}\right) d s & =\frac{1}{2} \int_{\varepsilon}^{t} U_{s}^{\delta} d s \\
& \leq-8 \int_{\varepsilon}^{t} s \partial_{s}\left(\chi_{k}^{2}, \Phi_{s}^{\delta}\right)_{\mu} d s+C_{3}(t-\varepsilon) \\
& =-\left.8 s\left(\chi_{k}^{2}, \Phi_{s}^{\delta}\right)_{\mu}\right|_{s=\varepsilon} ^{s=t}+8 \int_{\varepsilon}^{t}\left(\chi_{k}^{2}, \Phi_{s}^{\delta}\right)_{\mu} d s+C_{3}(t-\varepsilon) .
\end{aligned}
$$

Letting $\varepsilon \rightarrow 0$ and dividing by $t$, Lemma 4.4 gives that

$$
\mathscr{E}^{0}\left(\bar{\phi}_{t}^{\delta} \chi_{k}\right) \leq \frac{1}{t} \int_{0}^{t} \mathscr{E}^{0}\left(\phi_{s}^{\delta} \chi_{k}\right) d s \leq 16 K L+C_{3} .
$$

Therefore, $\left\{\mathscr{E}^{0}\left(\bar{\phi}_{t}^{\delta} \chi_{k}\right)\right\}_{0<t \leq T_{0}, 0<\delta \leq 1}$ is bounded. Moreover, since

$$
\begin{aligned}
& 2 \mathscr{E}^{0}\left(\Phi_{t}^{\delta} \chi_{k}\right) \\
& =\int_{E} \Gamma\left(\Phi_{t}^{\delta} \chi_{k}, \Phi_{t}^{\delta} \chi_{k}\right) d \mu \\
& =\int_{E}\left\{\Gamma\left(u_{t}^{\delta}-e_{t}^{\delta}\right)\left(\left(\phi^{\prime}\right)_{t}^{\delta}\right)^{4} \chi_{k}^{2}+2 \Gamma\left(u_{t}^{\delta}-e_{t}^{\delta}, \chi_{k}\right) \Phi_{t}^{\delta}\left(\left(\phi^{\prime}\right)_{t}^{\delta}\right)^{2} \chi_{k}+\Gamma\left(\chi_{k}\right)\left(\Phi_{t}^{\delta}\right)^{2}\right\} d \mu \\
& \leq 2 \int_{E} \Gamma\left(u_{t}^{\delta}-e_{t}^{\delta}\right)\left(\left(\phi^{\prime}\right)_{t}^{\delta}\right)^{4} \chi_{k}^{2} d \mu+2 \int_{E} \Gamma\left(\chi_{k}\right)\left(\Phi_{t}^{\delta}\right)^{2} d \mu \\
& \leq 2 W_{t}^{\delta}+2 K^{2} L^{2} a_{k} \quad \text { (from }(\text { C.3)) } \\
& \leq-16 t \partial_{t}\left(\chi_{k}^{2}, \Phi_{t}^{\delta}\right)_{\mu}+C_{3}+4 C_{2}+2 K^{2} L^{2} a_{k}, \quad \text { (from Eq. 4.10 and 4.11) }
\end{aligned}
$$

we can prove the boundedness of $\left\{\mathscr{E} 0\left(\bar{\Phi}_{t}^{\delta} \chi_{k}\right)\right\}_{0<t \leq T_{0}, 0<\delta \leq 1}$ in the same way.

\subsection{Sharper Estimates}

We write $u_{t}=-t \log T_{t} \mathbf{1}_{B}, \phi_{t}=\phi\left(u_{t}\right), \Phi_{t}=\Phi\left(u_{t}\right)$, and $\Psi_{t}=\Psi\left(u_{t}\right)$ for $t>0$. Since $\bar{\phi}_{t}^{\delta} \chi_{k}$ converges to $\bar{\phi}_{t} \chi_{k} \mu$-a.e. as $\delta \rightarrow 0$ and $\left\{\bar{\phi}_{t}^{\delta} \chi_{k}\right\}_{0 \leq t \leq T_{0}, 0<\delta \leq 1}$ is bounded in $\mathbb{D}$, we conclude that $\bar{\phi}_{t} \chi_{k} \in \mathbb{D}$ and $\left\{\bar{\phi}_{t} \chi_{k}\right\}_{0<t \leq T_{0}}$ is bounded in $\mathbb{D}$ for each $k$ by [13, Lemma 2.12]. Using the diagonal argument, for any decreasing sequence $\left\{t_{n}\right\} \downarrow 0$, we can find a subsequence $\left\{t_{n^{\prime}}\right\}$ such that, for every $k, \bar{\phi}_{t_{n^{\prime}}} \chi_{k}$ converges weakly to some $\psi_{k}$ in $\mathbb{D}$. Since $\chi_{k}=1$ on $\hat{E}_{l}$ when $k \geq l$, it follows that $\psi_{k}=\psi_{l} \mu$-a.e. on $\hat{E}_{l}$ for $k \geq l$. Therefore, there exists $\bar{\phi}_{0} \in \mathbb{D}_{\text {loc, } b}\left(\left\{\hat{E}_{k}\right\}\right)$ such that $\psi_{k}=\bar{\phi}_{0} \mu$-a.e. on $\hat{E}_{k}$ for every $k$.

We may also assume, by taking a further subsequence if necessary, that there exist $\Phi_{0}, \bar{\Phi}_{0}$, and $\bar{\Psi}_{0}$ in $L^{\infty}(\mu)$ such that $\Phi_{t_{n^{\prime}}} \rightarrow \Phi_{0}, \bar{\Phi}_{t_{n^{\prime}}} \rightarrow \bar{\Phi}_{0}, \bar{\Psi}_{t_{n^{\prime}}} \rightarrow \bar{\Psi}_{0}$ both in the weak$L^{2}(\tilde{\mu})$ sense and in the weak ${ }^{*}-L^{\infty}(\mu)$ sense. Here, $\tilde{\mu}$ is an arbitrary fixed finite measure on $E$ such that $\tilde{\mu}$ and $\mu$ are mutually absolutely continuous, and $L^{\infty}(\mu)$ is regarded as the dual space of $L^{1}(\mu)$. We remark that these functions depend on $K$. Because of this, it is more precise to write $\phi_{t}^{K}$ and $\bar{\Phi}_{0}^{K}$ instead of $\phi_{t}$ and $\bar{\Phi}_{0}$. 
Define

$$
Z_{t}=\left\{x \in E \mid T_{t} \mathbf{1}_{B}(x)-\mathbf{1}_{B}(x)>1\right\}
$$

for $t>0$. From the Chebyshev inequality, $\mu\left(Z_{t}\right) \leq\left\|T_{t} \mathbf{1}_{B}-\mathbf{1}_{B}\right\|_{2}^{2} \rightarrow 0$ as $t \rightarrow 0$; thus

$$
\frac{1}{t} \int_{0}^{t} \mathbf{1}_{Z_{s}} d s \rightarrow 0 \quad \text { in } L^{1}(\mu) \text { as } t \rightarrow 0 .
$$

For $x \in Z_{t}$,

$$
\phi_{t}(x)=\phi\left(-t \log T_{t} \mathbf{1}_{B}(x)\right)<\phi(-t \log 1)=0 .
$$

Since $T_{t} \mathbf{1}_{B}(x) \leq 2$ for $x \in E \backslash Z_{t}$ and $\phi(y) \geq y$ for $y \leq K$, it holds that

$$
\phi_{t}(x)=\phi\left(-t \log T_{t} \mathbf{1}_{B}(x)\right) \geq \phi(-t \log 2) \geq-t \log 2 \quad \text { on } E \backslash Z_{t} .
$$

Similar inequalities hold for $\Phi_{t}$ and $\Psi_{t}$.

Lemma 4.9 $\bar{\phi}_{0} \geq \bar{\Phi}_{0} \geq \bar{\Psi}_{0} \geq 0 \mu$-a.e. and $\Phi_{0} \geq 0 \mu$-a.e.

Proof By using (C.3) and the inequality (4.13) with $\phi_{t}$ replaced by $\Psi_{t}$, for $Y \in \mathscr{B}_{0}$,

$$
\begin{aligned}
\int_{Y} \bar{\Psi}_{t} d \mu & =\frac{1}{t} \int_{0}^{t}\left(\int_{Y \cap Z_{s}} \Psi_{s} d \mu+\int_{Y \backslash Z_{s}} \Psi_{s} d \mu\right) d s \\
& \geq-\frac{L K}{t} \int_{0}^{t} \mu\left(Z_{s}\right) d s-\frac{\mu(Y) \log 2}{2} t
\end{aligned}
$$

for every $t>0$. Then, by letting $t \rightarrow 0$ along the sequence $\left\{t_{n^{\prime}}\right\}$ in the above inequality, we obtain $\int_{Y} \bar{\Psi}_{0} d \mu \geq 0$ by applying Eq. 4.12 . Therefore, $\bar{\Psi}_{0} \geq 0 \mu$-a.e. Next, for $Y \in \mathscr{B}_{0}$,

$$
\begin{aligned}
\int_{Y}\left(\bar{\phi}_{t}-\bar{\Phi}_{t}\right) d \mu & =\frac{1}{t} \int_{0}^{t} \int_{Y \cap Z_{s}}\left(\phi_{s}-\Phi_{s}\right) d \mu d s+\frac{1}{t} \int_{0}^{t} \int_{Y \backslash Z_{s}}\left(\phi_{s}-\Phi_{s}\right) d \mu d s \\
& \geq-\frac{2 L K}{t} \int_{0}^{t} \mu\left(Z_{s}\right) d s+0 \quad(\text { from (C.3)) }
\end{aligned}
$$

for $t>0$ small enough. By the same argument, we obtain $\bar{\phi}_{0} \geq \bar{\Phi}_{0}$, and the other inequalities are proved in the same way.

Lemma 4.10 $\bar{\phi}_{0}=0 \mu$-a.e. on $B$.

Proof For an arbitrary sequence $\left\{s_{n}\right\} \downarrow 0, T_{s_{n}} \mathbf{1}_{B}$ converges to $\mathbf{1}_{B}$ in $L^{2}(\mu)$ as $n \rightarrow \infty$. Take an arbitrary subsequence $\left\{s_{n^{\prime}}\right\}$ from $\left\{s_{n}\right\}$. From this, we can find a subsequence $\left\{s_{n^{\prime \prime}}\right\}$ from $\left\{s_{n^{\prime}}\right\}$ such that $T_{S_{n^{\prime \prime}}} \mathbf{1}_{B} \rightarrow \mathbf{1}_{B} \mu$-a.e. as $n^{\prime \prime} \rightarrow \infty$. Using the dominated convergence theorem, $\lim _{n^{\prime \prime} \rightarrow \infty} \int_{B} \phi_{s_{n^{\prime \prime}}} d \mu=0$. This means $\lim _{t \rightarrow 0} \int_{B} \phi_{t} d \mu=0$. Then, by letting $t \rightarrow 0$ along the sequence $\left\{t_{n^{\prime}}\right\}$ in the identity

$$
\int_{B} \bar{\phi}_{t} d \mu=\frac{1}{t} \int_{0}^{t} \int_{B} \phi_{s} d \mu d s,
$$

we obtain $\int_{B} \bar{\phi}_{0} d \mu=0$. The claim follows directly.

Lemma 4.11 $\sqrt{\bar{\phi}_{0}} \in \mathbb{D}_{0}$. 
Proof Fix $h \in \mathbb{D}_{\hat{E}_{k}, b,+}$ arbitrarily. Since $\phi_{t}^{\delta} \chi_{k}-\left(\phi_{t}^{\delta}-\phi\left(e_{t}^{\delta}\right)\right)$ is constant on $\hat{E}_{k}$, Proposition 2.1 implies that

$$
\int_{E} \Gamma\left(\phi_{t}^{\delta} \chi_{k}\right) h d \mu=\int_{E} \Gamma\left(\phi_{t}^{\delta}-\phi\left(e_{t}^{\delta}\right)\right) h d \mu=\int_{E} \Gamma\left(u_{t}^{\delta}-e_{t}^{\delta}\right)\left(\left(\phi^{\prime}\right)_{t}^{\delta}\right)^{2} h d \mu .
$$

By using Lemma 4.6 with $\rho=h$,

$$
\begin{aligned}
& \int_{E} \Gamma\left(u_{t}^{\delta}-e_{t}^{\delta}\right)\left(\left(\phi^{\prime}\right)_{t}^{\delta}\right)^{2} h d \mu \\
& \leq-2 t\left(h, \partial_{t} \Phi_{t}^{\delta}\right)_{\mu}+2\left(h, \Psi_{t}^{\delta}\right)_{\mu}-2 t \mathscr{E}^{0}\left(\left(\left(\phi^{\prime}\right)_{t}^{\delta}\right)^{2} h, u_{t}^{\delta}-e_{t}^{\delta}\right) \\
& \quad+2 t\left(\frac{C t}{K}+1\right) \int_{E} \Gamma\left(u_{t}^{\delta}-e_{t}^{\delta}\right)\left(\left(\phi^{\prime}\right)_{t}^{\delta}\right)^{2} h d \mu+2 t^{2} \mathscr{E}^{0}(h)+2 t R(t, k) .
\end{aligned}
$$

Since

$$
\begin{aligned}
& -2 t \mathscr{E}^{0}\left(\left(\left(\phi^{\prime}\right)_{t}^{\delta}\right)^{2} h, u_{t}^{\delta}-e_{t}^{\delta}\right) \\
& =-t \int_{E} \Gamma\left(h, u_{t}^{\delta}-e_{t}^{\delta}\right)\left(\left(\phi^{\prime}\right)_{t}^{\delta}\right)^{2} d \mu-2 t \int_{E} \Gamma\left(u_{t}^{\delta}-e_{t}^{\delta}\right)\left(\phi^{\prime}\right)_{t}^{\delta}\left(\phi^{\prime \prime}\right)_{t}^{\delta} h d \mu \\
& \leq-2 t \mathscr{E}^{0}\left(h, \Phi_{t}^{\delta} \chi_{k}\right)+\frac{2 C t}{K} \int_{E} \Gamma\left(\phi_{t}^{\delta} \chi_{k}\right) h d \mu \quad \text { (from (C.2)), }
\end{aligned}
$$

we have

$$
\begin{aligned}
& \left(1-\frac{2 C t}{K}-\frac{2 C t^{2}}{K}-2 t\right) \int_{E} \Gamma\left(\phi_{t}^{\delta} \chi_{k}\right) h d \mu \\
& \leq-2 t\left(h, \partial_{t} \Phi_{t}^{\delta}\right)_{\mu}+2\left(h, \Psi_{t}^{\delta}\right)_{\mu}-2 t \mathscr{E}^{0}\left(h, \Phi_{t}^{\delta} \chi_{k}\right)+2 t^{2} \mathscr{E}^{0}(h)+2 t R(t, k) .
\end{aligned}
$$

Then, for $T \in\left(0, T_{0}\right]$,

$$
\begin{aligned}
& \frac{1}{2 T} \int_{0}^{T}\left(1-\frac{2 C T}{K}-\frac{2 C T^{2}}{K}-2 T\right) \int_{E} \Gamma\left(\phi_{t}^{\delta} \chi_{k}\right) h d \mu d t \\
& \leq-\frac{1}{T} \int_{0}^{T} t\left(h, \partial_{t} \Phi_{t}^{\delta}\right)_{\mu} d t+\left(h, \bar{\Psi}_{T}^{\delta}\right)_{\mu}-\frac{1}{T} \int_{0}^{T} t \mathscr{E}^{0}\left(h, \Phi_{t}^{\delta} \chi_{k}\right) d t+C_{4} T,
\end{aligned}
$$

where $C_{4}=T_{0} \mathscr{E}^{0}(h) / 3+R\left(T_{0}, k\right) / 2$. Integration by parts gives

$$
\frac{1}{T} \int_{0}^{T} t\left(h, \partial_{t} \Phi_{t}^{\delta}\right)_{\mu} d t=\frac{1}{T}\left(T\left(h, \Phi_{T}^{\delta}\right)_{\mu}-\int_{0}^{T}\left(h, \Phi_{t}^{\delta}\right)_{\mu} d t\right)=\left(h, \Phi_{T}^{\delta}\right)_{\mu}-\left(h, \bar{\Phi}_{T}^{\delta}\right)_{\mu}
$$

and

$$
\begin{aligned}
\frac{1}{T} \int_{0}^{T} t \mathscr{E}^{0}\left(h, \Phi_{t}^{\delta} \chi_{k}\right) d t & =\left.\frac{t}{T} \int_{0}^{t} \mathscr{E}^{0}\left(h, \Phi_{s}^{\delta} \chi_{k}\right) d s\right|_{t=0} ^{t=T}-\frac{1}{T} \int_{0}^{T} \int_{0}^{t} \mathscr{E}^{0}\left(h, \Phi_{s}^{\delta} \chi_{k}\right) d s d t \\
& =T \mathscr{E}^{0}\left(h, \bar{\Phi}_{T}^{\delta} \chi_{k}\right)-\frac{1}{T} \int_{0}^{T} t \mathscr{E}^{0}\left(h, \bar{\Phi}_{t}^{\delta} \chi_{k}\right) d t \\
& \rightarrow 0 \text { as } \delta \rightarrow 0 \text { and } T \rightarrow 0
\end{aligned}
$$


because $\mathscr{E}^{0}\left(\bar{\Phi}_{t}^{\delta} \chi_{k}\right)$ is bounded in $\delta$ and $t$ by Lemma 4.8. Letting $\delta \rightarrow 0$ and $T \rightarrow 0$ along the sequence $\left\{t_{n^{\prime}}\right\}$ in Eq. 4.14 , we obtain from Lemmas 4.3 and 4.4 that

$$
\frac{1}{2} \int_{E} \Gamma\left(\bar{\phi}_{0}\right) h d \mu \leq-\left(h, \Phi_{0}\right)_{\mu}+\left(h, \bar{\Phi}_{0}\right)_{\mu}+\left(h, \bar{\Psi}_{0}\right)_{\mu} \leq\left(2 \bar{\phi}_{0}, h\right)_{\mu} .
$$

The second inequality follows from Lemma 4.9. Then, for each $\varepsilon>0$,

$$
\int_{E} \Gamma\left(\sqrt{\bar{\phi}_{0}+\varepsilon}-\sqrt{\varepsilon}\right) h d \mu=\frac{1}{4} \int_{E} \Gamma\left(\bar{\phi}_{0}\right) \frac{h}{\bar{\phi}_{0}+\varepsilon} d \mu \leq \frac{1}{2}\left(2 \bar{\phi}_{0}, \frac{h}{\bar{\phi}_{0}+\varepsilon}\right) \leq\|h\|_{1} .
$$

This inequality holds for all $h \in \bigcup_{k=1}^{\infty} \mathbb{D}_{\hat{E}_{k}, b,+}$. Hence, $\left|D\left(\sqrt{\bar{\phi}_{0}+\varepsilon}-\sqrt{\varepsilon}\right)\right|_{H} \leq 1 \mu$ a.e. Next, fix $k \in \mathbb{N}$ and let $f_{\varepsilon}$ denote $\left(\sqrt{\bar{\phi}_{0}+\varepsilon}-\sqrt{\varepsilon}\right) \chi_{k}$ for $\varepsilon>0$. Then, from the argument above, $\left|D f_{\varepsilon}\right|_{H} \leq 1 \mu$-a.e. on $\hat{E}_{k}$ and $\left\{f_{\varepsilon}\right\}_{\varepsilon>0}$ is bounded in $\mathbb{D}$. Any weak limit in $\mathbb{D}$ of a subsequence should be $\sqrt{\bar{\phi}_{0}} \chi_{k}$, and so $\left|D\left(\sqrt{\bar{\phi}_{0}} \chi_{k}\right)\right|_{H} \leq 1 \mu$-a.e. on $\hat{E}_{k}$. Therefore, $\sqrt{\bar{\phi}_{0}} \in \mathbb{D}_{0}$.

From Lemma 4.10, Lemma 4.11, and Proposition 2.7, we conclude $\bar{\phi}_{0} \leq \mathrm{d}_{B}^{2} \mu$-a.e. The multiplicative constant can be further improved since this inequality is not sharp.

Lemma 4.12 Given $K>0$, we can choose $M>0$ such that

$$
\Phi^{K}\left(\phi_{t}^{M} \mathbf{1}_{E \backslash Z_{t}}\right) \geq \Psi_{t}^{K}, \quad t \in(0, K / \log 2] .
$$

Proof Since $\Phi^{K}$ is non-decreasing, $\Psi^{K} \leq \Phi^{K}$ on $[-K, \infty)$ and (C.5), we can find $M>0$ such that $\Phi^{K}(M) \geq \sup _{s \in \mathbb{R}} \Psi^{K}(s)$. From Eq. 4.13, $\phi_{t}^{M} \geq-t \log 2 \geq-K$ on $E \backslash Z_{t}$. Then, on $E \backslash Z_{t}$,

$$
\Phi^{K}\left(\phi_{t}^{M} \mathbf{1}_{E \backslash Z_{t}}\right)=\Phi^{K}\left(\phi_{t}^{M}\right) \geq \Phi^{K}\left(M \wedge u_{t}\right)=\Phi^{K}(M) \wedge \Phi^{K}\left(u_{t}\right) \geq \Psi^{K}\left(u_{t}\right) .
$$

On $Z_{t}$, Eq. 4.16 is trivial since the left-hand side is zero and the right-hand side is nonpositive.

\section{Lemma 4.13 If the inequality}

$$
\bar{\phi}_{0}^{K} \leq \beta \frac{\mathrm{d}_{B}^{2}}{2} \quad \mu \text {-a.e. on }\left\{\mathrm{d}_{B}<N\right\}
$$

is true for some $\beta>1$ for every $K>0$ and every limit $\bar{\phi}_{0}^{K}$, then

$$
\bar{\phi}_{0}^{K} \leq\left(2-\beta^{-1}\right) \frac{\mathrm{d}_{B}^{2}}{2} \quad \mu \text {-a.e. on }\left\{\mathrm{d}_{B}<N\right\} .
$$

Proof Given $K>0$, we take $M$ as in Lemma 4.12. Let $Y \in \mathscr{B}_{0}$ with $Y \subset\left\{\mathrm{d}_{B}<N\right\}$. Using the convexity of $\Phi^{K}(-t \log (\cdot))$ for small $t$, from Lemma 4.1, we have

$$
\left(\Phi_{t}^{K}, \mathbf{1}_{Y}\right)_{\mu}=\int_{Y} \Phi^{K}\left(-t \log T_{t} \mathbf{1}_{B}\right) d \mu \geq \mu(Y) \Phi^{K}\left(-t \log \left(\frac{1}{\mu(Y)}\left(T_{t} \mathbf{1}_{B}, \mathbf{1}_{Y}\right)_{\mu}\right)\right) .
$$

By the upper estimate (2.8),

$$
\varliminf_{t \rightarrow 0}-t \log \left(T_{t} \mathbf{1}_{B}, \mathbf{1}_{Y}\right)_{\mu}=\underline{\lim }_{t \rightarrow 0}-t \log P_{t}(Y, B) \geq \frac{\mathrm{d}(Y, B)^{2}}{2} .
$$


Therefore, in the limit,

$$
\begin{aligned}
\left(\Phi_{0}^{K}, \mathbf{1}_{Y}\right)_{\mu} & \geq \mu(Y) \Phi^{K}\left(\frac{\mathrm{d}(Y, B)^{2}}{2}\right) \\
& =\mu(Y) \underset{x \in Y}{\operatorname{essinf}} \Phi^{K}\left(\frac{\mathrm{d}_{B}(x)^{2}}{2}\right) \\
& \geq \frac{\mu(Y)}{\beta} \underset{x \in Y}{\operatorname{essinf}} \Phi^{K}\left(\bar{\phi}_{0}^{M}(x)\right)
\end{aligned}
$$

from the assumption of Eq. 4.17 and (C.6). Recall now the function $\hat{\Phi}^{K}$ that was defined in Eq. 4.1. Since $\hat{\Phi}^{K}$ is 1 -Lipschitz and concave on $\mathbb{R}$, for $t \in(0, K / \log 2]$ we have

$$
\begin{aligned}
\hat{\Phi}^{K}\left(\bar{\phi}_{t}^{M}\right) & =\hat{\Phi}^{K}\left(\frac{1}{t} \int_{0}^{t} \phi^{M}\left(u_{s}\right) \mathbf{1}_{E \backslash Z_{s}} d s+\frac{1}{t} \int_{0}^{t} \phi^{M}\left(u_{s}\right) \mathbf{1}_{Z_{s}} d s\right) \\
& \geq \hat{\Phi}^{K}\left(\frac{1}{t} \int_{0}^{t} \phi^{M}\left(u_{s}\right) \mathbf{1}_{E \backslash Z_{s}} d s\right)-\frac{1}{t}\left|\int_{0}^{t} \phi^{M}\left(u_{s}\right) \mathbf{1}_{Z_{s}} d s\right| \\
& \geq \frac{1}{t} \int_{0}^{t} \hat{\Phi}^{K}\left(\phi^{M}\left(u_{s}\right) \mathbf{1}_{E \backslash Z_{s}}\right) d s-\frac{L M}{t} \int_{0}^{t} \mathbf{1}_{Z_{s}} d s \\
& \geq \bar{\Psi}_{t}^{K}-\frac{L M}{t} \int_{0}^{t} \mathbf{1}_{Z_{s}} d s . \quad \text { (from Eq. } 4.13 \text { and Lemma 4.12) }
\end{aligned}
$$

Take $\left\{t_{n}\right\} \downarrow 0$ such that $\bar{\phi}_{t_{n}}^{M}$ converges to $\bar{\phi}_{0}^{M}$ weakly in $L^{2}(\tilde{\mu})$. From Lemma 4.9, Lemma 4.2, and Eq. 4.12, we obtain that $\Phi^{K}\left(\bar{\phi}_{0}^{M}\right)=\hat{\Phi}^{K}\left(\bar{\phi}_{0}^{M}\right) \geq \bar{\Psi}_{0}^{K} \mu$-a.e. Combining this inequality and Eq. 4.18, we get

$$
\left(\Phi_{0}^{K}, \mathbf{1}_{Y}\right)_{\mu} \geq \frac{\mu(Y)}{\beta} \underset{x \in Y}{\operatorname{essinf}} \bar{\Psi}_{0}^{K}(x) .
$$

We will prove

$$
\Phi_{0}^{K} \geq \beta^{-1} \bar{\Psi}_{0}^{K} \quad \mu \text {-a.e. on }\left\{\mathrm{d}_{B}<N\right\} .
$$

Assume for contradiction that there exists some $Y^{\prime} \in \mathscr{B}_{0}$ and $\varepsilon>0$ such that $Y^{\prime} \subset\left\{\mathrm{d}_{B}<\right.$ $N\}$ and $\Phi_{0}^{K}<\beta^{-1} \bar{\Psi}_{0}^{K}-\varepsilon$ on $Y^{\prime}$. Let

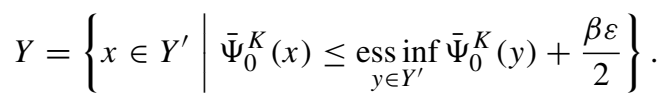

Then, $\mu(Y)>0$ and $\Phi_{0}^{K} \leq \beta^{-1} \operatorname{ess~inf}_{y \in Y} \bar{\Psi}_{0}^{K}(y)-\varepsilon / 2$ on $Y$. This contradicts Eq. 4.19.

Combining Eq. 4.20 with 4.15 and using Lemma 4.9, we obtain

$$
\frac{1}{2} \int_{E} \Gamma\left(\bar{\phi}_{0}^{K}\right) h d \mu \leq-\beta^{-1}\left(h, \bar{\Psi}_{0}^{K}\right)_{\mu}+\left(h, \bar{\Phi}_{0}^{K}\right)_{\mu}+\left(h, \bar{\Psi}_{0}^{K}\right)_{\mu} \leq\left(2-\beta^{-1}\right)\left(\bar{\phi}_{0}^{K}, h\right)_{\mu}
$$

for every $h$ expressed by $h=h_{1} h_{2}$, where $h_{1} \in \bigcup_{k=1}^{\infty} \mathbb{D}_{\hat{E}_{k}, b,+}$ and $h_{2}=\left(0 \vee k\left(N-\mathrm{d}_{B}\right)\right) \wedge 1$ for some $k \in \mathbb{N}$. The claim follows by the same argument after Eq. 4.15.

By repeated application of Lemma 4.13, $\bar{\phi}_{0} \leq \mathrm{d}_{B}^{2} / 2 \mu$-a.e. on $\left\{\mathrm{d}_{B}<N\right\}$, and so $\bar{\Phi}_{0} \leq$ $\mathrm{d}_{B}^{2} / 2 \mu$-a.e. on $\left\{\mathrm{d}_{B}<N\right\}$ from Lemma 4.9. To obtain the equality, we modify Lemmas 2.9 , 2.13 , and 2.14 of [9] as follows. 
Lemma 4.14 For any limit $\Phi_{0}$ (that is a weak- $L^{2}(\tilde{\mu})$ and weak ${ }^{*}-L^{\infty}(\mu)$-limit of a subsequence of $\left\{\Phi_{t}\right\}_{t>0}$ ),

$$
\Phi\left(\mathrm{d}_{B}^{2} / 2\right) \leq \Phi_{0} \quad \text { p-a.e. on }\left\{\mathrm{d}_{B}<N\right\} .
$$

Proof Let $Y \in \mathscr{B}_{0}$ such that $Y \subset\left\{\mathrm{d}_{B}<N\right\}$. From the upper estimate (2.8) and Lemma 4.1,

$$
\begin{aligned}
& \Phi\left(\frac{\mathrm{d}(Y, B)^{2}}{2}\right) \leq \Phi\left(\frac{\lim }{t \rightarrow 0}-t \log P_{t}(Y, B)\right) \\
& =\Phi\left(\frac{\lim }{t \rightarrow 0}-t \log \left(\frac{1}{\mu(Y)}\left(T_{t} \mathbf{1}_{B}, \mathbf{1}_{Y}\right)_{\mu}\right)\right) \\
& =\varliminf_{t \rightarrow 0} \Phi\left(-t \log \left(\frac{1}{\mu(Y)}\left(T_{t} \mathbf{1}_{B}, \mathbf{1}_{Y}\right)_{\mu}\right)\right) \\
& \leq \varliminf_{t \rightarrow 0} \frac{1}{\mu(Y)} \int_{Y} \Phi\left(-t \log T_{t} \mathbf{1}_{B}\right) d \mu \\
& =\varliminf_{t \rightarrow 0} \frac{1}{\mu(Y)} \int_{Y} \Phi_{t} d \mu \\
& \leq \frac{1}{\mu(Y)} \int_{Y} \Phi_{0} d \mu \text {. }
\end{aligned}
$$

Let $Y_{\varepsilon}=\left\{\Phi_{0} \leq \Phi\left(\mathrm{d}_{B}^{2} / 2\right)-\varepsilon\right\} \cap\left\{\mathrm{d}_{B}<N\right\}$ for $\varepsilon>0$ and suppose, for the sake of contradiction, that $\mu\left(Y_{\varepsilon}\right)>0$. Then, $Y_{\varepsilon}^{\prime}:=\left\{x \in Y_{\varepsilon} \cap E_{k} \mid \Phi\left(\mathrm{d}_{B}^{2}(x) / 2\right) \leq \Phi\left(\mathrm{d}\left(Y_{\varepsilon}, B\right)^{2} / 2\right)+\varepsilon / 2\right\}$ also has $\mu$-positive measure for sufficiently large $k$. But

$$
\begin{aligned}
\frac{1}{\mu\left(Y_{\varepsilon}^{\prime}\right)} \int_{Y_{\varepsilon}^{\prime}} \Phi\left(\frac{\mathrm{d}_{B}^{2}}{2}\right) d \mu & \leq \Phi\left(\frac{\mathrm{d}\left(Y_{\varepsilon}^{\prime}, B\right)^{2}}{2}\right)+\frac{\varepsilon}{2} \\
& \leq \frac{1}{\mu\left(Y_{\varepsilon}^{\prime}\right)} \int_{Y_{\varepsilon}^{\prime}} \Phi_{0} d \mu+\frac{\varepsilon}{2} \\
& \leq \frac{1}{\mu\left(Y_{\varepsilon}^{\prime}\right)} \int_{Y_{\varepsilon}^{\prime}} \Phi\left(\frac{\mathrm{d}_{B}^{2}}{2}\right) d \mu-\frac{\varepsilon}{2},
\end{aligned}
$$

which is a contradiction. Therefore, $\mu\left(Y_{\varepsilon}\right)=0$. Since $\varepsilon>0$ is arbitrary, we obtain the desired assertion.

Lemma 4.15 For any limit $\bar{\Phi}_{0}$ (that is, in particular, a weak- $L^{2}(\tilde{\mu})$ limit of a subsequence of $\left.\left\{\bar{\Phi}_{t}\right\}_{t>0}\right)$, $\Phi\left(\mathrm{d}_{B}^{2} / 2\right) \leq \bar{\Phi}_{0} \mu$-a.e. on $\left\{\mathrm{d}_{B}<N\right\}$.

Proof For any $\rho \in L^{2}(\tilde{\mu})$ with $\rho \geq 0 \mu$-a.e. and $\rho=0 \mu$-a.e. on $\left\{\mathrm{d}_{B}<N\right\}$, Lemma 4.14 implies

$$
\left(\Phi\left(\mathrm{d}_{B}^{2} / 2\right), \rho\right)_{\tilde{\mu}} \leq \underline{\lim }_{t \rightarrow 0}\left(\Phi_{t}, \rho\right)_{\tilde{\mu}} .
$$

Therefore,

$$
\left(\Phi\left(\mathrm{d}_{B}^{2} / 2\right), \rho\right)_{\tilde{\mu}} \leq \lim _{t \rightarrow 0} \frac{1}{t} \int_{0}^{t}\left(\Phi_{s}, \rho\right)_{\tilde{\mu}} d s=\varliminf_{t \rightarrow 0}\left(\bar{\Phi}_{t}, \rho\right)_{\tilde{\mu}} \leq\left(\bar{\Phi}_{0}, \rho\right)_{\tilde{\mu}} .
$$

This implies the desired claim. 
From Lemma 4.14 and (C.4), we have $\bar{\Phi}_{0}=\mathrm{d}_{B}^{2} / 2$ on $\left\{\mathrm{d}_{B}^{2} / 2 \leq K\right\} \cap\left\{\mathrm{d}_{B}<N\right\}=$ : $Y_{K, N}$, independently of the choice of subsequence. In particular, $\bar{\Phi}_{t} \mathbf{1}_{Y_{K, N}}$ converges to $\left(\mathrm{d}_{B}^{2} / 2\right) \mathbf{1}_{Y_{K, N}}$ weakly in $L^{2}(\tilde{\mu})$ as $t \rightarrow 0$. Furthermore, we have the following

Lemma 4.16 $\bar{\Phi}_{0}=\Phi\left(\mathrm{d}_{B}^{2} / 2\right)$ on $\left\{\mathrm{d}_{B}<N\right\}$.

Proof We specify the dependency on $K$ and write $\Phi_{t}^{K}$ instead of $\Phi_{t}$. From what we have proven, $\bar{\Phi}_{t}^{M} \mathbf{1}_{Y_{M, N}}$ converges to $\left(\mathrm{d}_{B}^{2} / 2\right) \mathbf{1}_{Y_{M, N}}$ weakly in $L^{2}(\tilde{\mu})$ and $\bar{\Phi}_{0}^{M}=\mathrm{d}_{B}^{2} / 2$ on $Y_{M, N}$ for all $M>0$. From (C.7) and the 1-Lipschitz continuity of $\Phi^{K}$,

$$
\begin{aligned}
\bar{\Phi}_{T}^{K} & =\frac{1}{T} \int_{0}^{T} \Phi^{K}\left(u_{t}\right) d t \\
& \leq \frac{1}{T} \int_{0}^{T} \Phi^{K}\left(\Phi^{M}\left(u_{t}\right)\right) d t+\left(\Phi^{K}(\infty)-\Phi^{K}(M)\right) \\
& \leq \frac{1}{T} \int_{0}^{T} \Phi^{K}\left(\Phi^{M}\left(u_{t}\right) \mathbf{1}_{E \backslash Z_{t}}\right) d t+\frac{1}{T} \int_{0}^{T}\left|\Phi^{M}\left(u_{t}\right) \mathbf{1}_{Z_{t}}\right| d t+\left(\Phi^{K}(\infty)-\Phi^{K}(M)\right) \\
& \leq \frac{1}{T} \int_{0}^{T} \Phi^{K}\left(\Phi^{M}\left(u_{t}\right) \mathbf{1}_{E \backslash Z_{t}}\right) d t+\frac{L M}{T} \int_{0}^{T} \mathbf{1}_{Z_{t}} d t+\left(\Phi^{K}(\infty)-\Phi^{K}(M)\right)
\end{aligned}
$$

Since $\hat{\Phi}^{K}$ is concave and 1-Lipschitz, the first term is estimated as follows:

$$
\begin{aligned}
\frac{1}{T} \int_{0}^{T} \Phi^{K}\left(\Phi^{M}\left(u_{t}\right) \mathbf{1}_{E \backslash Z_{t}}\right) d t & =\frac{1}{T} \int_{0}^{T} \hat{\Phi}^{K}\left(\Phi^{M}\left(u_{t}\right) \mathbf{1}_{E \backslash Z_{t}}\right) d t \\
& \leq \hat{\Phi}^{K}\left(\frac{1}{T} \int_{0}^{T} \Phi^{M}\left(u_{t}\right) \mathbf{1}_{E \backslash Z_{t}} d t\right) \\
& \leq \hat{\Phi}^{K}\left(\frac{1}{T} \int_{0}^{T} \Phi^{M}\left(u_{t}\right) d t\right)+\left|\frac{1}{T} \int_{0}^{T} \Phi^{M}\left(u_{t}\right) \mathbf{1}_{Z_{t}} d t\right| \\
& \leq \hat{\Phi}^{K}\left(\bar{\Phi}_{T}^{M}\right)+\frac{L M}{T} \int_{0}^{T} \mathbf{1}_{Z_{t}} d t .
\end{aligned}
$$

Combining Eqs. 4.21 and 4.22 and letting $T \rightarrow 0$ along a suitable subsequence, we have

$$
\bar{\Phi}_{0}^{K} \leq \hat{\Phi}^{K}\left(\mathrm{~d}_{B}^{2} / 2\right)+\left(\Phi^{K}(\infty)-\Phi^{K}(M)\right) \quad \text { on } Y_{M, N} .
$$

Here, we also used Lemma 4.2 and Eq. 4.12 for the right-hand side. Letting $M \rightarrow \infty$, we obtain $\bar{\Phi}_{0}^{K} \leq \Phi^{K}\left(\mathrm{~d}_{B}^{2} / 2\right)$ on $\left\{\mathrm{d}_{B}<N\right\}$. The inequality in the other direction has been proven already.

Therefore, $\bar{\Phi}_{t} \mathbf{1}_{\left\{\mathrm{d}_{B}<N\right\}}$ converges both in the weak- $L^{2}(\tilde{\mu})$ sense and in the weak ${ }^{*}-L^{\infty}(\mu)$ sense as $t \rightarrow 0$, and the limit $\bar{\Phi}_{0} \mathbf{1}_{\left\{\mathrm{d}_{B}<N\right\}}$ is equal to $\Phi\left(\mathrm{d}_{B}^{2} / 2\right) \mathbf{1}_{\left\{\mathrm{d}_{B}<N\right\}}$.

\subsection{Application of the Tauberian Theorem}

In the following, $\left\{T_{t}^{0}\right\}_{t>0}$ denotes the Markovian semigroup corresponding to the strong local Dirichlet form $(\mathscr{E}, \mathbb{D})$, and $\mathscr{L}^{0}$ denotes the generator of $\left\{T_{t}^{0}\right\}_{t>0}$ on $L^{2}(\mu)$. Note 
that $\mathscr{E}^{0}\left(T_{t}^{0} f\right) \leq(2 e t)^{-1}\|f\|_{2}^{2}$ holds for all $f \in L^{2}(\mu)$ and $t>0$, from the spectral decomposition theorem. For $k \in \mathbb{N}$, we define

$$
\hat{\chi}_{k}=\chi_{k} \cdot\left(\left(0 \vee k\left(N-\mathrm{d}_{B}\right)\right) \wedge 1\right) \in \mathbb{D}_{E_{k} \cap\left\{\mathrm{d}_{B}<N\right\}, b} .
$$

We use $(\cdot, \cdot)_{\hat{\chi}_{k}^{2} \cdot \mu}$ to denote the $L^{2}$-inner product with respect to the measure $\hat{\chi}_{k}^{2} \cdot \mu$ on $E$.

Lemma 4.17 For $\tau \in(0,1)$ and $Y \in \mathscr{B}_{0}$,

$$
\lim _{t \rightarrow 0}\left(T_{\tau-t}^{0} \mathbf{1}_{Y}, \Phi_{t}\right)_{\hat{\chi}_{k}^{2} \cdot \mu}=\left(T_{\tau}^{0} \mathbf{1}_{Y}, \bar{\Phi}_{0}\right)_{\hat{\chi}_{k}^{2} \cdot \mu} \text {. }
$$

Proof Let $f(t)=\left(T_{\tau-t}^{0} \mathbf{1}_{Y}, \Phi_{t}-\Phi(\infty)\right)_{\hat{\chi}_{k}^{2} \cdot \mu}$ for $t \in(0, \tau)$. We will confirm the following two conditions:

(i) $T^{-1} \int_{0}^{T} f(s) d s$ converges to $\left(T_{\tau}^{0} \mathbf{1}_{Y}, \bar{\Phi}_{0}-\Phi(\infty)\right)_{\hat{\chi}_{k}^{2} \cdot \mu}$ as $T \rightarrow 0$,

(ii) there exist $M>0$ and $t_{0} \in(0, \tau)$ such that $f(t)-f(s) \leq M(t-s) / s$ for any $0<s<t \leq t_{0}$.

Under these conditions, we can apply Wiener's Tauberian theorem (see, e.g., [16, Lemma 3.11]) to obtain $\lim _{t \rightarrow 0} f(t)=\left(T_{\tau}^{0} \mathbf{1}_{Y}, \bar{\Phi}_{0}-\Phi(\infty)\right)_{\hat{\chi}_{k}^{2} \cdot \mu}$. Combining this equality and the identity

$$
\lim _{t \rightarrow 0}\left(T_{\tau-t}^{0} \mathbf{1}_{Y}, \Phi(\infty)\right)_{\hat{\chi}_{k}^{2} \cdot \mu}=\left(T_{\tau}^{0} \mathbf{1}_{Y}, \Phi(\infty)\right)_{\hat{\chi}_{k}^{2} \cdot \mu},
$$

we obtain the desired claim.

Condition (i) is proved as follows:

$$
\begin{aligned}
& \left|\frac{1}{T} \int_{0}^{T} f(t) d t-\left(T_{\tau}^{0} \mathbf{1}_{Y}, \bar{\Phi}_{0}-\Phi(\infty)\right)_{\hat{\chi}_{k}^{2} \cdot \mu}\right| \\
& \leq \frac{1}{T} \int_{0}^{T}\left|\left(T_{\tau-t}^{0} \mathbf{1}_{Y}-T_{\tau}^{0} \mathbf{1}_{Y}, \Phi_{t}-\Phi(\infty)\right)_{\hat{\chi}_{k}^{2} \cdot \mu}\right| d t+\left|\left(T_{\tau}^{0} \mathbf{1}_{Y}, \bar{\Phi}_{T}-\bar{\Phi}_{0}\right)_{\hat{\chi}_{k}^{2} \cdot \mu}\right| \\
& \leq \frac{2 K L}{T} \int_{0}^{T}\left\|\mathbf{1}_{Y}-T_{t}^{0} \mathbf{1}_{Y}\right\|_{1} d t+\left|\left(T_{\tau}^{0} \mathbf{1}_{Y}, \bar{\Phi}_{T}-\bar{\Phi}_{0}\right)_{\hat{\chi}_{k}^{2} \cdot \mu}\right| \\
& \rightarrow 0 \quad \text { as } T \rightarrow 0 .
\end{aligned}
$$

Here, we used the fact that $\left\{T_{t}^{0}\right\}_{t>0}$ extends to a strongly continuous semigroup on $L^{1}(\mu)$. For condition (ii), we fix $\delta \in(0,1)$. The function $f^{\delta}(r):=\left(T_{\tau-r}^{0} \mathbf{1}_{Y}, \Phi_{r}^{\delta}-\Phi\left(e_{r}^{\delta}\right)\right)_{\hat{\chi}_{k}^{2} \cdot \mu}$ is continuously differentiable on $(0, \tau)$ and

$$
\begin{aligned}
\frac{d}{d r} f^{\delta}(r) & =\left(T_{\tau-r}^{0} \mathbf{1}_{Y}, \partial_{r} \Phi_{r}^{\delta}-\partial_{r} \Phi\left(e_{r}^{\delta}\right)\right)_{\hat{\chi}_{k}^{2} \cdot \mu}+\left(\partial_{r} T_{\tau-r}^{0} \mathbf{1}_{Y}, \Phi_{r}^{\delta}-\Phi\left(e_{r}^{\delta}\right)\right)_{\hat{\chi}_{k}^{2} \cdot \mu} \\
& =\left(T_{\tau-r}^{0} \mathbf{1}_{Y}, \partial_{r} \Phi_{r}^{\delta}\right)_{\hat{\chi}_{k}^{2} \cdot \mu}-\frac{1}{r}\left(T_{\tau-r}^{0} \mathbf{1}_{Y}, \Psi\left(e_{r}^{\delta}\right)\right)_{\hat{\chi}_{k}^{2} \cdot \mu}-\left(\mathscr{L}^{0} T_{\tau-r}^{0} \mathbf{1}_{Y}, \Phi_{r}^{\delta}-\Phi\left(e_{r}^{\delta}\right)\right)_{\hat{\chi}_{k}^{2} \cdot \mu} \\
& \leq\left(T_{\tau-r}^{0} \mathbf{1}_{Y}, \partial_{r} \Phi_{r}^{\delta}\right)_{\hat{\chi}_{k}^{2} \cdot \mu}+\mathscr{E}^{0}\left(T_{\tau-r}^{0} \mathbf{1}_{Y}, \hat{\chi}_{k}^{2}\left(\Phi_{r}^{\delta}-\Phi\left(e_{r}^{\delta}\right)\right)\right)=: J_{1}+J_{2} .
\end{aligned}
$$


Let $r \in(0, \tau / 2]$ and $Q_{r}=\int_{E} \Gamma\left(u_{r}^{\delta}-e_{r}^{\delta}\right)\left(\left(\phi^{\prime}\right)_{r}^{\delta}\right)^{2} \hat{\chi}_{k}^{2} T_{\tau-r}^{0} \mathbf{1}_{Y} d \mu$. By using Lemma 4.6 with $f=\mathbf{1}_{B}$ and $\rho=\hat{\chi}_{k}^{2} T_{\tau-r}^{0} \mathbf{1}_{Y}$,

$$
\begin{aligned}
J_{1} \leq \frac{1}{r}\left(\hat{\chi}_{k}^{2} T_{\tau-r}^{0} \mathbf{1}_{Y}, \Psi_{r}^{\delta}\right)_{\mu}-\mathscr{E}^{0}\left(\left(\left(\phi^{\prime}\right)_{r}^{\delta}\right)^{2} \hat{\chi}_{k}^{2} T_{\tau-r}^{0} \mathbf{1}_{Y}, u_{r}^{\delta}-e_{r}^{\delta}\right) \\
\quad-\frac{1}{2 r} \int_{E} \Gamma\left(u_{r}^{\delta}-e_{r}^{\delta}\right)\left(\left(\phi^{\prime}\right)_{r}^{\delta}\right)^{2} \hat{\chi}_{k}^{2} T_{\tau-r}^{0} \mathbf{1}_{Y} d \mu \\
\quad+\left(\frac{C r}{K}+1\right) \int_{E} \Gamma\left(u_{r}^{\delta}-e_{r}^{\delta}\right)\left(\left(\phi^{\prime}\right)_{r}^{\delta}\right)^{2} \hat{\chi}_{k}^{2} T_{\tau-r}^{0} \mathbf{1}_{Y} d \mu+r \mathscr{E}^{0}\left(\hat{\chi}_{k}^{2} T_{\tau-r}^{0} \mathbf{1}_{Y}\right)+R(r, k) \\
\leq \frac{C_{5}}{r}-\mathscr{E}^{0}\left(\left(\left(\phi^{\prime}\right)_{r}^{\delta}\right)^{2} \hat{\chi}_{k}^{2} T_{\tau-r}^{0} \mathbf{1}_{Y}, u_{r}^{\delta}-e_{r}^{\delta}\right)+\left(\frac{C r}{K}+1-\frac{1}{2 r}\right) Q_{r}+C_{6},
\end{aligned}
$$

where $C_{5}=K L \mu(Y)$ and $C_{6}=(\tau / 2) \sup _{s \in[\tau / 2, \tau)} \mathscr{E}^{0}\left(\hat{\chi}_{k}^{2} T_{s}^{0} \mathbf{1}_{Y}\right)+R(\tau / 2, k)$. We also have

$$
\begin{aligned}
\mathscr{E} 0\left(\left(\left(\phi^{\prime}\right)_{r}^{\delta}\right)^{2} \hat{\chi}_{k}^{2} T_{\tau-r}^{0} \mathbf{1}_{Y}, u_{r}^{\delta}-e_{r}^{\delta}\right)= & \frac{1}{2} \int_{E}\left(\left(\phi^{\prime}\right)_{r}^{\delta}\right)^{2} \hat{\chi}_{k}^{2} \Gamma\left(T_{\tau-r}^{0} \mathbf{1}_{Y}, u_{r}^{\delta}-e_{r}^{\delta}\right) d \mu \\
& +\int_{E} \hat{\chi}_{k}\left(\left(\phi^{\prime}\right)_{r}^{\delta}\right)^{2} T_{\tau-r}^{0} \mathbf{1}_{Y} \Gamma\left(\hat{\chi}_{k}, u_{r}^{\delta}-e_{r}^{\delta}\right) d \mu \\
& +\int_{E} \hat{\chi}_{k}^{2}\left(\phi^{\prime}\right)_{r}^{\delta}\left(\phi^{\prime \prime}\right)_{r}^{\delta} T_{\tau-r}^{0} \mathbf{1}_{Y} \Gamma\left(u_{r}^{\delta}-e_{r}^{\delta}\right) d \mu \\
=: & J_{3}+J_{4}+J_{5} .
\end{aligned}
$$

Accordingly,

$$
\frac{d}{d r} f^{\delta}(r) \leq J_{2}-J_{3}-J_{4}-J_{5}+\frac{C_{5}}{r}+\left(\frac{C r}{K}+1-\frac{1}{2 r}\right) Q_{r}+C_{6}
$$

Then,

$$
\begin{aligned}
J_{2}-J_{3} & =\frac{1}{2} \int_{E} \Gamma\left(T_{\tau-r}^{0} \mathbf{1}_{Y}, \hat{\chi}_{k}^{2}\right)\left(\Phi_{r}^{\delta}-\Phi\left(e_{r}^{\delta}\right)\right) d \mu \\
& \leq 2 K L \mathscr{E}^{0}\left(T_{\tau-r}^{0} \mathbf{1}_{Y}\right)^{1 / 2} \mathscr{E}^{0}\left(\hat{\chi}_{k}^{2}\right)^{1 / 2} \\
& \leq 4 K L(e \tau)^{-1 / 2} \mu(Y)^{1 / 2} \mathscr{E}^{0}\left(\hat{\chi}_{k}\right)^{1 / 2}=: C_{7}, \\
\left|J_{4}\right| \leq \frac{1}{2} \int_{E}\left\{\Gamma\left(\hat{\chi}_{k}\right)\right. & \left.+\hat{\chi}_{k}^{2}\left(\left(\phi^{\prime}\right)_{r}^{\delta}\right)^{2} \Gamma\left(u_{r}^{\delta}-e_{r}^{\delta}\right)\right\} T_{\tau-r}^{0} \mathbf{1}_{Y} d \mu \leq \mathscr{E}^{0}\left(\hat{\chi}_{k}\right)+\frac{Q_{r}}{2},
\end{aligned}
$$

and

Therefore, we have

$$
\left|J_{5}\right| \leq \frac{C}{K} Q_{r}
$$

$$
\frac{d}{d r} f^{\delta}(r) \leq\left(\frac{C+C r}{K}+\frac{3}{2}-\frac{1}{2 r}\right) Q_{r}+\frac{C_{5}}{r}+C_{6}+C_{7}+\mathscr{E} 0\left(\hat{\chi}_{k}\right) .
$$

Let $C_{8}$ denote $C_{6}+C_{7}+\mathscr{E}^{0}\left(\hat{\chi}_{k}\right)$. If $r \leq t_{0}:=\min \left\{(2(C \tau+C) / K+3)^{-1}, \tau / 2\right\}$, then we have $\frac{d}{d r} f^{\delta}(r) \leq C_{5} / r+C_{8}$. From this,

$$
f^{\delta}(t)-f^{\delta}(s) \leq \int_{s}^{t}\left(\frac{C_{5}}{r}+C_{8}\right) d r \leq \frac{C_{5}+C_{8} t_{0}}{s}(t-s), \quad 0<s<t \leq t_{0} .
$$

By letting $\delta \rightarrow 0$, we obtain the desired assertion.

Let $\tau>0, k \in \mathbb{N}$, and $Y \in \mathscr{B}_{0}$. It holds that

$$
\int_{Y} \hat{\chi}_{k}^{2} \Phi_{t} d \mu=\left(\Phi_{t}, T_{\tau-t}^{0} \mathbf{1}_{Y}\right)_{\hat{\chi}_{k}^{2} \cdot \mu}+\left(\hat{\chi}_{k}^{2} \Phi_{t}, \mathbf{1}_{Y}-T_{\tau-t}^{0} \mathbf{1}_{Y}\right)_{\mu}
$$


for every $t \in(0, \tau]$. From Lemma 4.17,

$$
\lim _{\tau \rightarrow 0} \lim _{t \rightarrow 0}\left(\Phi_{t}, T_{\tau-t}^{0} \mathbf{1}_{Y}\right)_{\hat{\chi}_{k}^{2} \cdot \mu}=\left(\bar{\Phi}_{0}, \mathbf{1}_{Y}\right)_{\hat{\chi}_{k}^{2} \cdot \mu} .
$$

For the second term of Eq. 4.23, we have

$$
\lim _{\tau \rightarrow 0} \lim _{t \rightarrow 0}\left(\hat{\chi}_{k}^{2} \Phi_{t}, \mathbf{1}_{Y}-T_{\tau-t}^{0} \mathbf{1}_{Y}\right)_{\mu}=0
$$

by the boundedness of $\Phi_{t}$ and the strong continuity of $\left\{T_{t}^{0}\right\}$. Therefore,

$$
\lim _{t \rightarrow 0} \int_{Y} \hat{\chi}_{k}^{2} \Phi_{t} d \mu=\int_{Y} \hat{\chi}_{k}^{2} \bar{\Phi}_{0} d \mu=\int_{Y} \hat{\chi}_{k}^{2} \Phi\left(\mathrm{d}_{B}^{2} / 2\right) d \mu .
$$

Using this equality, we finish the proof of Theorem 2.11. For $\varepsilon \in(0,(N-\mathrm{d}(A, B)) / 2)$, define $A_{\varepsilon}=\left\{x \in A \mid \mathrm{d}_{B}(x) \leq \mathrm{d}(A, B)+\varepsilon\right\}$. Then, for sufficiently large $k, \hat{\chi}_{k}=\chi_{k}=1$ $\mu$-a.e. on $A_{\varepsilon} \cap \hat{E}_{k}$. Using the convexity of $\Phi^{K}(-t \log (\cdot))$ for small $t$ (Lemma 4.1), we have

$$
\begin{aligned}
\varlimsup_{t \rightarrow 0} \Phi\left(-t \log P_{t}(A, B)\right) & \leq \varlimsup_{t \rightarrow 0} \Phi\left(-t \log P_{t}\left(A_{\varepsilon}, B\right)\right) \\
& \leq \varlimsup_{t \rightarrow 0} \frac{1}{\mu\left(A_{\varepsilon}\right)} \int_{A_{\varepsilon}} \Phi\left(-t \log T_{t} \mathbf{1}_{B}\right) d \mu \\
& =\varlimsup_{t \rightarrow 0} \frac{1}{\mu\left(A_{\varepsilon}\right)}\left(\int_{A_{\varepsilon} \cap \hat{E}_{k}} \hat{\chi}_{k}^{2} \Phi_{t} d \mu+\int_{A_{\varepsilon} \backslash \hat{E}_{k}} \Phi_{t} d \mu\right) \\
& \leq \varlimsup_{t \rightarrow 0} \frac{1}{\mu\left(A_{\varepsilon}\right)} \int_{A_{\varepsilon}} \hat{\chi}_{k}^{2} \Phi_{t} d \mu+\frac{K L}{\mu\left(A_{\varepsilon}\right)} \mu\left(A_{\varepsilon} \backslash \hat{E}_{k}\right) \\
& =\frac{1}{\mu\left(A_{\varepsilon}\right)} \int_{A_{\varepsilon}} \hat{\chi}_{k}^{2} \Phi\left(\frac{\mathrm{d}_{B}^{2}}{2}\right) d \mu+\frac{K L}{\mu\left(A_{\varepsilon}\right)} \mu\left(A_{\varepsilon} \backslash \hat{E}_{k}\right) \quad \text { (from Eq. 4.24) } \\
& \leq \frac{(\mathrm{d}(A, B)+\varepsilon)^{2}}{2}+\frac{K L}{\mu\left(A_{\varepsilon}\right)} \mu\left(A_{\varepsilon} \backslash \hat{E}_{k}\right) .
\end{aligned}
$$

Letting $k \rightarrow \infty$, we have

$$
\varlimsup_{t \rightarrow 0} \Phi\left(-t \log P_{t}(A, B)\right) \leq \frac{(\mathrm{d}(A, B)+\varepsilon)^{2}}{2} .
$$

Since $\varepsilon$ is arbitrary and $\lim _{K \rightarrow \infty} \Phi^{K}(x)=x$ for each $x \in \mathbb{R}$, Eq. 2.9 holds. This completes the proof of Theorem 2.11 .

\section{Proof of Auxiliary Propositions and Examples}

We prove Propositions 2.9 and 2.14.

Proof (of Proposition 2.9) Let $Y=\left\{\mathrm{d}_{B}=\infty\right\}$. From [1, Proposition 5.1], $Y$ coincides with $\left\{T_{t}^{0} \mathbf{1}_{B}=0\right\}$ up to $\mu$-null sets for all $t>0$. Then, for $t>0$ and $f \in L^{2}(\mu)$ with $f \geq 0$ $\mu$-a.e.,

$$
\left(T_{t}^{0}\left(\mathbf{1}_{Y} f\right), T_{1}^{0} \mathbf{1}_{B}\right)_{\mu}=\left(\mathbf{1}_{Y} f, T_{t+1}^{0} \mathbf{1}_{B}\right)_{\mu}=0 .
$$


This implies that

$$
T_{t}^{0}\left(\mathbf{1}_{Y} f\right)=0 \quad \mu \text {-a.e. on } E \backslash Y
$$

because $T_{1}^{0} \mathbf{1}_{B}>0 \mu$-a.e. on $E \backslash Y$. Equation 5.1 now holds for all $f \in L^{2}(\mu)$; thus, $Y$ is an invariant set with respect to $(\mathscr{E} 0, \mathbb{D})$, from [6, Lemma 1.6.1]. From [6, Theorem 1.6.1], $\mathbf{1}_{Y} f \in \mathbb{D}$ for every $f \in \mathbb{D}$ and

$$
\mathscr{E}^{0}(f, g)=\mathscr{E}^{0}\left(\mathbf{1}_{Y} f, \mathbf{1}_{Y} g\right)+\mathscr{E}^{0}\left(\mathbf{1}_{E \backslash Y} f, \mathbf{1}_{E \backslash Y} g\right), \quad f, g \in \mathbb{D} .
$$

Moreover, $D\left(\mathbf{1}_{Y} f\right)=\mathbf{1}_{Y} D f$ by Proposition 2.1. By using these properties, we can confirm that $\mathscr{E}\left(\mathbf{1}_{E \backslash Y} f, \mathbf{1}_{Y} g\right)=0$ for $f, g \in \mathbb{D}$. From [10, Lemma 3.1], $E \backslash Y$ is weakly invariant with respect to $(\mathscr{E}, \mathbb{D})$. That is, $T_{t}\left(\mathbf{1}_{E \backslash Y} f\right)=0 \mu$-a.e. on $Y$ for all $t>0$ and $f \in L^{2}(\mu)$. Letting $f=\mathbf{1}_{B}$, we obtain $T_{t} \mathbf{1}_{B}=0 \mu$-a.e. on $Y$ since $B \subset E \backslash Y$. Thus, for $A \in \mathscr{B}_{0}$ satisfying $\mathrm{d}(A, B)=\infty$, we have $\mu(A \backslash Y)=0$ and so $P_{t}(A, B)=0$ holds.

Proof (of Proposition 2.14) Let $f \in \bigcup_{k=1}^{\infty} \mathbb{D}_{E_{k}, b}$ with $\|f\|_{2}=1$. For $x \geq 0$ and $y \geq 0$,

$$
x y \leq \int_{0}^{x} \log ^{+} s d s+\int_{0}^{y} e^{t} d t \leq \int_{0}^{x} \log ^{+} x d s+e^{y}-1=x \log ^{+} x+e^{y}-1 .
$$

Applying this inequality to $x=f^{2}$ and $y=\delta\left|b_{2}-c_{2}\right|_{H}^{2}$ and integrating it provides

$$
\delta \int_{E}\left|b_{2}-c_{2}\right|_{H}^{2} f^{2} d \mu \leq \int_{E} f^{2} \log ^{+} f^{2} d \mu+\int_{E}\left(e^{\delta\left|b_{2}-c_{2}\right|_{H}^{2}}-1\right) d \mu .
$$

Then,

$$
\begin{aligned}
\int_{E}\left|b_{2}-c_{2}\right|_{H} f^{2} d \mu & \leq\left(\int_{E}\left|b_{2}-c_{2}\right|_{H}^{2} f^{2} d \mu\right)^{1 / 2} \\
& \leq \delta^{-1 / 2}\left(\int_{E} f^{2} \log ^{+} f^{2} d \mu\right)^{1 / 2}+\delta^{-1 / 2}\left\{\int_{E}\left(e^{\delta\left|b_{2}-c_{2}\right|_{H}^{2}}-1\right) d \mu\right\}^{1 / 2} .
\end{aligned}
$$

Thus, (B. $\left.2^{\prime}\right)$ holds with $\gamma=\delta^{-1 / 2}$ and

$$
\lambda_{\varepsilon}=\hat{\lambda}_{\varepsilon}+\delta^{-1 / 2}\left\{\int_{E}\left(e^{\delta\left|b_{2}-c_{2}\right|_{H}^{2}}-1\right) d \mu\right\}^{1 / 2}
$$

for $\varepsilon>0$.

We discuss other sufficient conditions of Assumption 2.8, (B.1), (B.2) ${ }_{A, B}$, and (B.2').

For $\alpha>0$, let $\mathscr{T}_{\alpha}$ denote the set of all real measurable functions $\psi$ on $E$ satisfying the following requirement: there exists a measurable nest $\left\{E_{k}\right\}_{k=1}^{\infty}$ and $\tilde{\lambda}_{\alpha} \geq 0$ such that $\psi \in L_{\mathrm{loc}}^{1}\left(\mu,\left\{E_{k}\right\}\right)$ and

$$
\int_{E}|\psi| f^{2} d \mu \leq \alpha \mathscr{E}^{0}(f)+\tilde{\lambda}_{\alpha}\|f\|_{2}^{2}, \quad f \in \bigcup_{k=1}^{\infty} \mathbb{D}_{E_{k}, b} .
$$

Also, let $\mathscr{T}_{0+}=\bigcap_{\alpha>0} \mathscr{T}_{\alpha}$ and $\mathscr{T}_{\infty}=\bigcup_{\alpha>0} \mathscr{T}_{\alpha}$. Clearly, $L^{\infty}(\mu) \subset \mathscr{T}_{0+}$. 
Lemma 5.1 Let a be an $H$-valued measurable function on $E$, and $\alpha>0$. If $|a|_{H}^{2} \in \mathscr{T}_{\alpha}$ with a measurable nest $\left\{E_{k}\right\}_{k=1}^{\infty}$ and $\tilde{\lambda}_{\alpha} \geq 0$, then the following inequalities hold for $f, g \in$ $\bigcup_{k=1}^{\infty} \mathbb{D}_{E_{k}, b}$ and $\varepsilon>0$ :

$$
\begin{aligned}
\left|\int_{E}(a, D f)_{H} g d \mu\right| & \leq\left(2 \mathscr{E}^{0}(f)\right)^{1 / 2}\left(\alpha \mathscr{E}^{0}(g)+\tilde{\lambda}_{\alpha}\|g\|_{2}^{2}\right)^{1 / 2}, \\
\left|\int_{E}(a, D f)_{H} f d \mu\right| & \leq \sqrt{2 \alpha} \mathscr{E}^{0}(f)+\frac{\tilde{\lambda}_{\alpha}}{\sqrt{2 \alpha}}\|f\|_{2}^{2} \\
\int_{E}|a|_{H} f^{2} d \mu & \leq \varepsilon \mathscr{E}^{0}(f)+\left(\frac{\varepsilon \tilde{\lambda}_{\alpha}}{\alpha}+\frac{\alpha}{4 \varepsilon}\right)\|f\|_{2}^{2} .
\end{aligned}
$$

Proof Equation 5.3 holds by combining the inequality

$$
\left|\int_{E}(a, D f)_{H} g d \mu\right| \leq\left(\int_{E}|D f|_{H}^{2} d \mu\right)^{1 / 2}\left(\int_{E}|a|_{H}^{2} g^{2} d \mu\right)^{1 / 2}
$$

with Eq. 5.2. By letting $g=f$,

$$
\begin{aligned}
\left|\int_{E}(a, D f)_{H} f d \mu\right| & \leq\left(2 \mathscr{E}^{0}(f)\right)^{1 / 2}\left(\alpha \mathscr{E}^{0}(f)+\tilde{\lambda}_{\alpha}\|f\|_{2}^{2}\right)^{1 / 2} \\
& \leq \frac{\sqrt{2 \alpha}}{4} \cdot 2 \mathscr{E}^{0}(f)+\frac{1}{\sqrt{2 \alpha}}\left(\alpha \mathscr{E}^{0}(f)+\tilde{\lambda}_{\alpha}\|f\|_{2}^{2}\right),
\end{aligned}
$$

which proves Eq. 5.4. Equation 5.5 follows from the following calculation:

$$
\begin{aligned}
\int_{E}|a|_{H} f^{2} d \mu & \leq \frac{\varepsilon}{\alpha} \int_{E}|a|_{H}^{2} f^{2} d \mu+\frac{\alpha}{4 \varepsilon} \int_{E} f^{2} d \mu \\
& \leq \frac{\varepsilon}{\alpha}\left(\alpha \mathscr{E} 0(f)+\tilde{\lambda}_{\alpha}\|f\|_{2}^{2}\right)+\frac{\alpha}{4 \varepsilon}\|f\|_{2}^{2} .
\end{aligned}
$$

We can give sufficient conditions for Assumption 2.8, (B.1), and (B.2') in terms of $\mathscr{T}_{\alpha}$.

Proposition 5.2 Suppose $|b|_{H},|c|_{H} \in L_{\text {loc }}^{2}\left(\mu,\left\{E_{k}\right\}\right)$ and $V \in L_{\text {loc }}^{1}\left(\mu,\left\{E_{k}\right\}\right)$ for some measurable nest $\left\{E_{k}\right\}_{k=1}^{\infty}$. Let $V_{+}$denote $V \vee 0$ and $V_{-}$denote $(-V) \vee 0$.

(i) If $|b+c|_{H}^{2} \in \mathscr{T}_{\alpha_{1}},|b-c|_{H}^{2} \in \mathscr{T}_{\alpha_{2}}, V_{+} \in \mathscr{T}_{\alpha_{3}}, V_{-} \in \mathscr{T}_{\alpha_{4}}$ with $\alpha_{i}>0(i=1,2,3,4)$ and $\sqrt{2 \alpha_{1}}+\alpha_{4}<1$, then (A.2) holds.

(ii) If $|b-c|_{H}^{2} \in \mathscr{T}_{\infty}$, then (B.1) holds.

(iii) If $b$ and $c$ are decomposed into $b=b_{1}+b_{2}$ and $c=c_{1}+c_{2}$, respectively, such that $\left|b_{1}-c_{1}\right|_{H}^{2} \in \mathscr{T}_{0+}$ and $\exp \left(\delta\left|b_{2}-c_{2}\right|_{H}^{2}\right)-1 \in L^{1}(\mu)$ for some $\delta>0$, then $\left(B .2^{\prime}\right)$ holds.

Proof (i): Equation 2.2 follows from Eq. 5.4. Since

$$
|b|_{H}^{2}+|c|_{H}^{2}=\left(|b+c|_{H}^{2}+|b-c|_{H}^{2}\right) / 2,
$$

we see that $|b|_{H}^{2},|c|_{H}^{2} \in \mathscr{T}_{\left(\alpha_{1}+\alpha_{2}\right) / 2}$. Moreover, $|V| \in \mathscr{T}_{\alpha_{3}+\alpha_{4}}$. Combining these and Eq. 5.3, we obtain Eq. 2.3.

(ii): This follows from Eq. 5.4. 
(iii): From Eq. 5.5, Eq. 2.11 holds with $\hat{\lambda}_{\varepsilon}=\inf _{\alpha>0}\left(\frac{\varepsilon \tilde{\lambda}_{\alpha}}{\alpha}+\frac{\alpha}{4 \varepsilon}\right)$ for $\varepsilon>0$. Moreover, for any $\alpha>0$,

$$
\varlimsup_{\varepsilon \rightarrow 0} \varepsilon \hat{\lambda}_{\varepsilon} \leq \varlimsup_{\varepsilon \rightarrow 0} \varepsilon\left(\frac{\varepsilon \tilde{\lambda}_{\alpha}}{\alpha}+\frac{\alpha}{4 \varepsilon}\right)=\frac{\alpha}{4},
$$

which implies that $\varlimsup_{\varepsilon \rightarrow 0} \varepsilon \hat{\lambda}_{\varepsilon}=0$. Therefore, (B.2') holds, from Proposition 2.14.

The following are alternative descriptions of (B.2) ${ }_{A, B}$ and (B.2').

Proposition 5.3 (B.2) ${ }_{A, B}$ is equivalent to the following condition:

$(\tilde{B} .2)_{A, B} \quad$ There exist $\gamma \geq 0$ and a nonnegative and non-decreasing function $\Omega$ on $[0, \infty)$ such that $\lim _{x \rightarrow \infty} \Omega(x) / x=0$ and

$$
\begin{aligned}
& \int_{\left\{0<\mathrm{d}_{B}<\mathrm{d}(A, B)\right\}}\left(b-c, D \mathrm{~d}_{B}\right)_{H} f^{2} d \mu \leq \Omega\left(\mathscr{E}^{0}(f)^{1 / 2}\right)+\gamma\left(\int_{E} f^{2} \log ^{+} f^{2} d \mu\right)^{1 / 2} \\
& \text { for any } N>0 \text { and } f \in \bigcup_{k=1}^{\infty} \mathbb{D}_{E_{k}, b} \text { with }\|f\|_{2}=1 \text {. }
\end{aligned}
$$

Moreover, (B.2') is equivalent to the following condition:

$\left(\tilde{\mathrm{B}} .2^{\prime}\right) \quad$ There exist $\gamma \geq 0$ and a nonnegative and non-decreasing function $\Omega$ on $[0, \infty)$ such that $\lim _{x \rightarrow \infty} \Omega(x) / x=0$ and

$$
\int_{E}|b-c|_{H} f^{2} d \mu \leq \Omega\left(\mathscr{E}^{0}(f)^{1 / 2}\right)+\gamma\left(\int_{E} f^{2} \log ^{+} f^{2} d \mu\right)^{1 / 2}
$$

for any $f \in \bigcup_{k=1}^{\infty} \mathbb{D}_{E_{k}, b}$ with $\|f\|_{2}=1$.

Proof Suppose (B.2) $)_{A, B}$ holds. For $t \geq 0$, we define $\Omega(t)=\inf _{\varepsilon>0}\left(\varepsilon t^{2}+\lambda_{\varepsilon}\right)$. (B.2) $)_{A, B}$ implies Eq. 5.6. Moreover, $\Omega$ is non-decreasing by definition. For any $\alpha>0$, we have

$$
\frac{\Omega(t)}{t}=\inf _{\varepsilon>0}\left(\varepsilon t+\frac{\lambda_{\varepsilon}}{t}\right) \leq \alpha+\frac{\lambda_{\alpha / t}}{t} \stackrel{t \rightarrow \infty}{\longrightarrow} \alpha .
$$

Since $\alpha>0$ is arbitrary, we conclude that $\Omega(t) / t$ converges to 0 as $t \rightarrow \infty$. Thus, $(\tilde{\mathrm{B}} .2)_{A, B}$ holds.

Conversely, assume $(\tilde{\mathrm{B}} .2)_{A, B}$. Define $\lambda_{\varepsilon}=\sup _{t>0}\left(\Omega\left(t^{1 / 2}\right)-\varepsilon t\right)$ for each $\varepsilon>0$. Then, Eq. 2.7 holds. Furthermore, for any $\alpha>0$, there exists some $T>0$ such that $\Omega\left(t^{1 / 2}\right) / t^{1 / 2} \leq \alpha$ for all $t \geq T$. Then,

$$
\begin{aligned}
\varepsilon \lambda_{\varepsilon} & \leq \sup _{t \in[0, T)}\left(\varepsilon \Omega\left(t^{1 / 2}\right)-\varepsilon^{2} t\right) \vee \sup _{t \in[T, \infty)}\left(\varepsilon \Omega\left(t^{1 / 2}\right)-\varepsilon^{2} t\right) \\
& \leq \varepsilon \Omega\left(T^{1 / 2}\right) \vee \sup _{t \in[T, \infty)}\left(\varepsilon \alpha t^{1 / 2}-\varepsilon^{2} t\right) \\
& \leq \varepsilon \Omega\left(T^{1 / 2}\right) \vee\left(\alpha^{2} / 4\right) .
\end{aligned}
$$

Letting $\varepsilon \rightarrow 0$ and $\alpha \rightarrow 0$, we have $\lim _{\varepsilon \rightarrow 0} \varepsilon \lambda_{\varepsilon}=0$. Therefore, (B.2) $)_{A, B}$ holds.

The equivalence of $\left(\mathrm{B} .2^{\prime}\right)$ and $\left(\tilde{\mathrm{B}} .2^{\prime}\right)$ is proved in the same way.

We discuss some typical examples of non-symmetric forms that satisfy Assumption 2.8, (B.1), and (B.2'). 
Example 5.4 We assume the Sobolev inequality: for some $d>2$ and $S \geq 0$,

$$
\|f\|_{2 d /(d-2)}^{2} \leq S \mathscr{E}_{1}^{0}(f), \quad f \in \mathbb{D} .
$$

A typical example is the following: $E=\mathbb{R}^{d} ; \mu=d x$ (Lebesgue measure); $\mathbb{D}=H^{1}\left(\mathbb{R}^{d}\right.$ ) (the first-order $L^{2}$-Sobolev space); and $D=A(x) \nabla$, where $A(\cdot)$ is an $\mathbb{R}^{d \times d}$-valued measurable function on $E$ such that there exist $C>0$ satisfying $C^{-1} I \leq{ }^{t} A(x) A(x) \leq C I$ for $\mu$-a.e. $x$ in the quadratic form sense.

Let $\psi \in L^{d / 2}(\mu)+L^{\infty}(\mu)$. That is, let $\psi=\hat{\psi}+\check{\psi}$ for some $\hat{\psi} \in L^{d / 2}(\mu)$ and $\check{\psi} \in L^{\infty}(\mu)$. Take any $\varepsilon>0$. By using Eq. 5.7, for any $f \in \mathbb{D}$,

$$
\begin{aligned}
\int_{E}|\hat{\psi}| f^{2} d \mu & \leq\left\|\mathbf{1}_{\{|\hat{\psi}|>N\}} \hat{\psi}\right\|_{d / 2}\|f\|_{2 d /(d-2)}^{2}+N \int_{\{|\hat{\psi}| \leq N\}} f^{2} d \mu \\
& \leq S\left\|\mathbf{1}_{\{|\hat{\psi}|>N\}} \hat{\psi}\right\|_{d / 2} \mathscr{E}_{1}^{0}(f)+N\|f\|_{2}^{2}
\end{aligned}
$$

for any $N>0$. Take $N$ large enough that $S\left\|\mathbf{1}_{\{|\hat{\psi}|>N\}} \hat{\psi}\right\|_{d / 2} \leq \varepsilon$. Then,

$$
\int_{E}|\psi| f^{2} d \mu \leq \varepsilon \mathscr{E}^{0}(f)+\left(\varepsilon+N+\|\check{\psi}\|_{\infty}\right)\|f\|_{2}^{2} .
$$

Therefore, $L^{d / 2}(\mu)+L^{\infty}(\mu) \subset \mathscr{T}_{0+}$. In particular, due to Proposition 5.2, each of Assumption 2.8, (B.1), and (B.2') with $\gamma=0$ is satisfied if $|b|_{H},|c|_{H} \in L^{d}(\mu)+L^{\infty}(\mu)$ and $V \in L^{d / 2}(\mu)+L^{\infty}(\mu)$. In this case, the logarithmic term in (B. $\left.2^{\prime}\right)$ is not useful.

Example 5.5 Assume that $\mu(E)<\infty$ and that $\left(\mathscr{E}^{0}, \mathbb{D}\right)$ satisfies the defective logarithmic Sobolev inequality:

$$
\int_{E} f^{2} \log \left(f^{2} /\|f\|_{2}^{2}\right) d \mu \leq \alpha \mathscr{E}^{0}(f)+\beta\|f\|_{2}^{2}, \quad f \in \mathbb{D} .
$$

A typical example is the following: $(E, H, \mu)$ is an abstract Wiener space, $D$ is the $H$ derivative in the sense of the Malliavin calculus, and $\mathbb{D}$ is the first-order $L^{2}$ Sobolev space. In this case, we can take $\alpha=4$ and $\beta=0$.

Let $\psi \in L^{0}(\mu)$. By the Hausdorff-Young inequality $s t \leq s \log s-s+e^{t}$ for $s \geq 0$ and $t \in \mathbb{R}$

$$
\delta|\psi| g^{2} \leq g^{2} \log g^{2}-g^{2}+e^{\delta|\psi|} \quad \text { for } \delta>0 \text { and } g \in L^{2}(\mu) .
$$

Taking $g=f /\|f\|_{2}$ for $f \in \mathbb{D}$ and using Eq. 5.8, we have

$$
\int_{E}|\psi| f^{2} d \mu \leq \frac{\alpha}{\delta} \mathscr{E}^{0}(f)+\frac{\beta-1+\left\|e^{\delta|\psi|}\right\|_{1}}{\delta}\|f\|_{2}^{2} .
$$

Therefore, $|\psi| \in \mathscr{T}_{\alpha / \delta}$ if $e^{\delta|\psi|} \in L^{1}(\mu)$. Assumption 2.8, (B.1), and (B.2') hold if

$$
e^{\delta_{1}|b+c|_{H}^{2}}, e^{\delta_{2}|b-c|_{H}^{2}}, e^{\delta_{3} V_{+}}, e^{\delta_{4} V_{-}} \in L^{1}(\mu)
$$

with $\delta_{i}>0(i=1,2,3,4)$ and $\sqrt{2 \alpha / \delta_{1}}+\alpha / \delta_{4}<1$, by applying Proposition 5.2 with $b_{1}=0, b_{2}=b, c_{1}=0$, and $c_{2}=c$. We cannot expect that $\left(\mathrm{B} .2^{\prime}\right)$ will hold with $\gamma=0$ in general; thus, the introduction of the logarithmic term in $\left(\mathrm{B} .2^{\prime}\right)$ is effective in this case.

Acknowledgements This study was supported by JSPS KAKENHI Grant Number JP15H03625.

Open Access This article is distributed under the terms of the Creative Commons Attribution 4.0 International License (http://creativecommons.org/licenses/by/4.0/), which permits unrestricted use, distribution, and reproduction in any medium, provided you give appropriate credit to the original author(s) and the source, provide a link to the Creative Commons license, and indicate if changes were made. 


\section{References}

1. Ariyoshi, T., Hino, M.: Small-time asymptotic estimate in local Dirichlet spaces. Electron. J. Probab. 10, 1236-1259 (2005)

2. Bauer, H.: Measure and integration theory. de Gruyter Studies in Mathematics, vol. 26. Walter de Gruyter, Berlin (2001)

3. Bouleau, N., Hirsch, F.: Dirichlet forms and analysis on Wiener Space. de Gruyter Studies in Mathematics, vol. 14. Walter de Gruyter, Berlin (1991)

4. Davies, E.B.: Heat kernels and spectral theory. Cambridge Tracts in Mathematics, vol. 92. Cambridge University Press, Cambridge (1990)

5. Fitzsimmons, P.J., Kuwae, K.: Non-symmetric perturbations of symmetric Dirichlet forms. J. Funct. Anal. 208, 140-162 (2004)

6. Fukushima, M., Oshima, Y., Takeda, M.: Dirichlet forms and symmetric Markov processes, 2nd edn. de Gruyter Studies in Mathematics, vol. 19. Walter de Gruyter, Berlin (2011)

7. Hino, M.: Existence of invariant measures for diffusion processes on a Wiener space. Osaka J. Math. 35, 717-734 (1998)

8. Hino, M.: On short time asymptotic behavior of some symmetric diffusions on general state spaces. Potential Anal. 16, 249-264 (2002)

9. Hino, M., Ramírez, J.A.: Small-time Gaussian behavior of symmetric diffusion semigroups. Ann. Probab. 14, 1254-1295 (2003)

10. Kuwae, K.: Invariant sets and ergodic decomposition of local semi-Dirichlet forms. Forum Math. 23, 1259-1279 (2011)

11. Liskevich, V.: On $C_{0}$-semigroups generated by elliptic second order differential expressions on $L^{p}$ spaces. Differ. Integral Equ. 9, 811-826 (1996)

12. Lunt, J., Lyons, T.J., Zhang, T.S.: Integrability of functionals of Dirichlet processes, probabilistic representations of semigroups, and estimates of heat kernels. J. Funct. Anal. 153, 320-342 (1998)

13. Ma, Z.M., Röckner, M.: Introduction to the Theory of (Non-symmetric) Dirichlet Forms. Springer, Berlin (1992)

14. Ma, Z.M., Röckner, M.: Markov processes associated with positivity preserving coercive forms. Canad. J. Math. 47, 817-840 (1995)

15. Norris, J.R.: Heat kernel asymptotics and the distance function in Lipschitz Riemannian manifolds. Acta Math. 179, 79-103 (1997)

16. Ramírez, J.A.: Short time asymptotics in Dirichlet spaces. Comm. Pure Appl. Math. 54, 259-293 (2001)

17. Röckner, M., Zhang, T.S.: Probabilistic representations and hyperbound estimates for semigroups. Infin. Dimens. Anal. Quantum. Probab. Relat. Top. 2, 337-358 (1999)

18. Sobol, Z., Vogt, H.: On the $L_{p}$-theory of $C_{0}$-semigroups associated with second order elliptic operators. I. J. Funct. Anal. 193, 24-54 (2002)

19. Varadhan, R.: On the behavior of the fundamental solution of the heat equation with variable coefficients. Comm. Pure Appl. Math. 20, 431-455 (1967)

20. Voigt, J.: One-parameter semigroups acting simultaneously on different $L^{p}$ spaces. Bull. Soc. Roy. Sci. Liège 61, 465-470 (1992) 